\title{
Regulation of anti-apoptotic pathways in skeletal muscle and liver of an estivating species, Xenopus laevis.
}

\author{
By \\ Yulia Maistrovski \\ B.Sc. Carleton University, 2011
}

A thesis submitted to the Faculty of Graduate Studies and Research in partial fulfillment of the requirements for the degree of

\author{
Master of Science \\ Department Biology
}

Carleton University

Ottawa, Ontario, Canada 
The undersigned hereby recommend to the Faculty of Graduate Studies and Research acceptance of this thesis

\section{Regulation of anti-apoptotic pathways in skeletal muscle and liver of an estivating species, Xenopus laevis.}

Submitted by

\section{Yulia Maistrovski}

In partial fulfillment of the requirements for the degree of Master of Science

Chair, Department of Biology

Thesis Supervisor

Carleton University 
Abstract

This study investigated the anti-apoptotic pathways activated during dehydration and estivation of the African clawed frog, Xenopus laevis. Staying immobile for a significant period of time can cause serious tissue atrophy in non-adapted animals. To preserve skeletal muscle and liver mass, certain metabolic pathways with functions in cell preservation and protection must be activated. This study focused on two families of transcription factors, NF- $\kappa \mathrm{B}$ and STAT whose target genes include those with antiapoptotic functions. The data indicated an upregulation of the NF-KB pathway in liver of dehydrated frogs along with downstream targets Bcl-xL and c-IAP. STAT3 appeared to be active in liver whereas STAT5 was active in skeletal muscle. Relative levels of the $\mathrm{NF}-\kappa \mathrm{B}$ and STAT downstream target Bcl-2 were elevated in response to dehydration. Analysis of pro- and anti-apoptotic microRNAs indicated that these contribute to posttranscriptional regulation of mRNA transcripts that encode proteins with roles in cell survival. 


\section{Acknowledgments}

I would like to thank my supervisor, Kenneth Storey for his support and guidance throughout my project. I would also like to thank Jan Storey for editing this manuscript and for her continued support.

Thank you to my lab mates that could always be trusted for advice and encouragement. Finally, a special thank you for NSERC and OGS for funding my research. 
Table of Contents

$\begin{array}{ll}\text { Title Page } & \text { Page } \\ \text { Acceptance Sheet } & \text { i } \\ \text { Abstract } & \text { ii } \\ \text { Acknowledgements } & \text { iii } \\ \text { Table of Contents } & \text { iv } \\ \text { List of Abbreviations } & \text { v } \\ \text { List of Figures } & \text { vi } \\ \text { Lit of Tables } & \text { ix } \\ \text { Chapter 1: General Introduction } & \text { xiii } \\ \text { Chapter 2: Methods and Materials } & 1 \\ \text { Chapter 3: NF-אB Regulation } & 22 \\ \text { Chapter 4: STAT Regulation } & 39 \\ \text { Chapter 5: MiRNA regulation } & 72 \\ \text { Chapter 6: General Discussion } & 107 \\ \text { Appendix A } & 141\end{array}$




\section{List of Abbreviations}

2'-5' oligoadenylate synthetase

OAS

3 '-untranslated region

3'UTR

53-induced nuclear protein 1

TP53INP

$\alpha_{2}$-macroglobulin

$\alpha 2 \mathrm{MG}$

Acute myelogenous leukemia

AML

Aldo-keto reductase

AKR

Apolipoprotein L6

APOL6

Apoptosis inducing factor

AIF

Apoptotic protease activating factor-1

Asaf-1

Asparate-specific cysteine proteases

Caspases

Baculoviral IAP repeat

BIR

B-cell activating factor belonging to the TNF family

BAFF

B-cell lymphoma-2

Bcl-2

B-cell lymphoma-extra-large

Bcl-xl

Casein kinase II

CKII

Cell division cycle 25 homolog A

$\operatorname{cdc} 25 \mathrm{a}$

Chronic lymphocytic leukemia

CLL

Ciliary neurotrophic factor

$\mathrm{CNTF}$

Cross sectional area

CSA

Death-associated protein kinase 3

DAPK3

Enzyme-Linked Immunosorbent Assay

ELISA

Epidermal growth factor

EGF

Epidermal Growth Factor Receptor

EGRF

Erythropoietin

EPO

Eukaryotic translation initiation factor $4 \mathrm{E}$

eIF4E

Ezrin, radixin and moesin

ERM

Forkhead box protein $\mathrm{O} 1$

FOXO1

Gamma interferon activation site

GAS

Glutathione-S-transferase

GST

Hepatic stellate cells

HSC 
High Dehydration

Inhibitors of apoptosis $\quad$ IAPs

Inhibitors of NF- $\kappa \mathrm{B} \quad \mathrm{I} \kappa \mathrm{B}$

Interferon regulatory factor $1 \quad$ IRF1

Interferon Stimulated Response Element ISRE

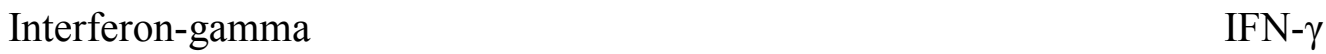

Interferon-stimulated gene factor $3 \quad$ ISGF-3

Interleukin

Interleukin-1 IL-1

IאB kinase $\quad$ IKK

Janus Kinases $\quad$ JAKs

Leukemia inhibitory factor LIF

$\begin{array}{ll}\text { Lymphotoxin- } \beta \text { receptor } & \text { LT } \beta R\end{array}$

Major histocompatibility complex $\quad$ MHC

Mammalian target of rapamycin mTOR

Matrix metalloproteinase $\quad$ MMP

Medium Dehydration $\quad$ MD

Metabolic rate depression $\quad$ MRD

Metalloproteinases $\quad$ MMPs

MicroRNA MiRNA

miRNA-induced silencing complex RISC

$\begin{array}{ll}\text { Myeloid cell leukaemia-1 } & \text { Mcl-1 }\end{array}$

Nuclear factor I/B NFIB

Nuclear Factor- $\kappa$ B $\quad$ NF- $\kappa$ B

$\begin{array}{ll}\text { Oncostatin M } & \text { OSM }\end{array}$

p38 mitogen-activated protein kinases $\quad$ p38 MAPK

Paired box $\quad$ PAX

$\begin{array}{ll}\text { Pathogen-associated molecular patterns } & \text { PAMPs }\end{array}$

$\begin{array}{ll}\text { Phosphatase and tensin } & \text { PTEN }\end{array}$

$\begin{array}{ll}\text { Phosphatidylinositol 3-kinase } & \text { PI3K }\end{array}$

Platelet-derived growth factor $\quad$ PDGF

Poly (ADP-ribose) polymerase PARP 
Precursor-miRNA

Programmed cell death protein 4

Protein kinase A

Protein kinase $\mathrm{R}$

Reverse transcription polymerase chain reaction

Reversion inducing cysteine-rich protein with kazal

RNA polymerase II

Signal Transducers and Activators of Transcription

Superoxide dismutase

T helper

Thrombopoietin

Toll-like receptor

Tropomyosin alpha-1 chain

Tumor necrosis factor

Tumor necrosis factor receptor

Vascular endothelial growth factor
pre-miRNA

PDCD4

PKA

PKR

RT-PCR

RECK

RNA pol II

STAT

MnSOD

TH

TPO

TLR

TPM1

TNF

TNFR

VEGF 


\section{List of Figures}

\# $\quad$ Title

1.1 The intrinsic and extrinsic apoptotic pathway.

3.1 The classical and alternative signaling pathways of NF- $\mathrm{KB}$ activation

3.2 Relative changes in protein expression of NF- $\kappa \mathrm{B}$ transcription factor subunits, p65 and p50, and their phosphorylated forms, p65 (Ser 529) and p50 (Ser 337) in liver tissue during medium and high dehydration of $X$. laevis

3.3 Relative protein levels of p65 (Ser 529) and p50 (Ser 337) in nuclear extracts of liver from control and high dehydration $X$. laevis.

3.4 Relative changes in DNA binding of NF- $\mathrm{kB}$ subunits, p65(Ser 529) and p50 (Ser 337 ), compared in nuclear extracts of control and highly dehydrated $X$. laevis liver samples.

3.5 Electrophoretic Mobility Shift Assay (EMSA) gel run with different amounts (40 $\mu \mathrm{g}, 32 \mu \mathrm{g}$ or $16 \mu \mathrm{g})$ of liver nuclear protein extract from both control (C) and high dehydration (HD) conditions.

3.6 Relative changes in mRNA transcript levels of NF- $\kappa \mathrm{B}$ downstream targets; cIAP1, Bcl-xL and p53 in liver in response to medium and high dehydration of $X$. laevis.

3.7 Relative changes in protein levels of NF- $\kappa \mathrm{B}$ downstream targets; c-IAP1, Bcl-xL 65 and p53 in liver tissues during medium and high dehydration of $X$. laevis.

3.8 Relative changes in the protein levels of NF- $\kappa \mathrm{B}$ transcription factor subunits, $\mathrm{p} 50 \quad 66$ and p65, and their phosphorylated forms, p50 (Ser 337) and p65 (Ser 529), in $X$. laevis skeletal muscle from medium and high dehydration conditions.

3.9 Relative protein levels of p50 (Ser 337) and p65 (Ser 529) in nuclear extracts of $X$. laevis skeletal muscle from control and high dehydration conditions.

3.10 Relative changes in mRNA transcript levels of NF- $\kappa \mathrm{B}$ downstream targets; cIAP1, Bcl-xL and p53 in skeletal muscle under medium and high dehydration of X. laevis.

3.11 Relative changes in protein levels of NF- $\kappa \mathrm{B}$ downstream targets; c-IAP1, Bcl-xL and p53 in X. laevis skeletal muscle from medium and high dehydration conditions.

4.1 The activation of the STAT pathway. 
4.2 Relative changes in protein expression of phosphorylated STAT transcription factors, STAT1 (Tyr 701), STAT2 (Tyr 690), STAT3 (Tyr 705), STAT3 (Ser 727), STAT5 (TYR 694) and STAT6 (Tyr 641), in liver tissue during medium and high dehydration of $X$. laevis.

4.3 Relative protein levels of STAT3 (Tyr 705) and STAT3 (Ser 727) in nuclear extracts of liver from control and high dehydration $X$. laevis.

4.4 Relative changes in DNA binding of STAT3 (Tyr 705) compared in nuclear 96 extracts of control and highly dehydrated $X$. laevis liver samples.

4.5 Electrophoretic Mobility Shift Assay (EMSA) gel of liver nuclear protein extract 97 from both control (C) and high dehydration (HD) conditions.

4.6 Relative changes in mRNA transcript levels of STAT3 downstream targets; bcl-2 98 and mcl-1 in liver in response to medium and high dehydration of $X$. laevis.

4.7 Relative changes in protein levels of STAT3 downstream targets; total bcl-2 in addition to bcl-2 (Thr 56) and bcl-2 (Ser 70) in liver tissues during medium and high dehydration of $X$. laevis.

4.8 Relative changes in the protein levels of STAT transcription factors, STAT1 (Tyr 100 701), STAT2 (Tyr 690), STAT3 (Tyr 705), STAT3 (Ser 727), STAT5 (TYR 694) and STAT6 (Tyr 641), in X. laevis skeletal muscle from medium and high dehydration conditions.

4.9 Relative protein levels of STAT5 (Tyr 694) in nuclear extracts of liver from control, medium dehydration, and high dehydration X. laevis.

4.10 Relative changes in DNA binding of STAT5 (Tyr 694) compared in nuclear extracts of control, medium dehydrated, and highly dehydrated $X$. laevis muscle samples.

4.11 Relative changes in mRNA transcript levels of STAT5 downstream targets; bcl-2 103 and mcl-1 in skeletal muscle under medium and high dehydration of $X$. laevis.

4.12 Relative changes in protein levels of STAT5 downstream targets; total bcl-2 in 104 addition to bcl-2 (Thr 56) and bcl-2 (Ser 70) in X. laevis skeletal muscle from medium and high dehydration conditions

5.1 MiRNA biosynthesis.

5.2 Relative changes in the transcript levels of three pro-apoptotic miRNAs, mir-15a, 127 mir-16 and mir-101, in liver in response to medium and high dehydration of $X$. laevis. 
5.3 Relative changes in the transcript levels of four anti-apoptotic miRNAs, mir-19b, 128 mir-21, mir-92a, and mir-155, in liver in response to medium and high dehydration of $X$. laevis.

5.4 Relative changes in the transcript levels of three pro-apoptotic miRNAs, mir-15a, 129 mir-16 and mir-101, in skeletal muscle in response to medium and high dehydration of $X$. laevis.

5.5 Relative changes in the transcript levels of four anti-apoptotic miRNAs, mir-19b, 130 mir-21, mir-92a, and mir-155, in skeletal muscle in response to medium and high dehydration of $X$. laevis.

5.6 Conservation analysis of the mir-15 and mir-16 binding sequence in the 3' UTR of $b c l-2$ and predicted binding structures of Xenopus specific mir-15a and mir-16 to the 3'UTR of $b c l-2$ with predicted binding energies.

5.7 Conservation analysis of the mir-101 binding sequence in the 3' UTR of $\mathrm{mcl}-1$ and predicted binding structures of Xenopus specific mir-101 to the 3'UTR of mcl-1 with predicted binding energies.

5.8 Conservation analysis of the mir-19b binding sequence in the 3' UTR of pten and predicted binding structures of Xenopus specific mir-19b to the 3'UTR of pten with predicted binding energies.

5.9 Conservation analysis of the mir-21 binding sequence in the 3' UTR of $p d c d 4$ and predicted binding structures of Xenopus specific mir-21 to the 3'UTR of $p d c d 4$ with predicted binding energies.

5.10 Conservation analysis of the mir-92a binding sequence in the 3' UTR of bim and predicted binding structures of Xenopus specific mir-92a to the 3'UTR of bim with predicted binding energies.

5.11 Conservation analysis of the mir-155 binding sequence in the 3' UTR of tp53inp and predicted binding structures of Xenopus specific mir-155 to the 3'UTR of tp53inp with predicted binding energies.

6.1 Summary figure of the relative changes in mRNA transcript levels of the 152 combined NF- $\kappa$ B and STAT downstream targets; c-IAP, Bcl-xL, bcl-2 and mcl-1 in liver in response to medium and high dehydration of $X$. laevis.

6.2 Summary figure of the relative changes in protein levels of the combined NF- $\kappa \mathrm{B}$ and STAT downstream targets; c-IAP, Bcl-xL, total bcl-2 in addition to bcl-2 (Thr 56) and bcl-2 (Ser 70) in liver tissues during medium and high dehydration of $X$. laevis. 
6.3 Summary figure of the relative changes in mRNA transcript levels of the combined NF- $\kappa$ B and STAT downstream targets; c-IAP, Bcl-xL, bcl-2 and mcl-1 in skeletal muscle in response to medium and high dehydration of $X$. laevis.

6.4 Summary figure of the relative changes in protein levels of the combined NF- $\kappa \mathrm{B}$ and STAT downstream targets; c-IAP, Bcl-xL, total bcl-2 in addition to bcl-2 (Thr 56) and bcl-2 (Ser 70) in skeletal muscle tissues during medium and high dehydration of $X$. laevis.

6.5 Summary figure of the relative changes in the transcript levels of the proapoptotic miRNAs, mir-15a and mir-16, along with the relative changes in mRNA and protein levels of their target gene, $b c l-2$.

6.6 Summary figure of the relative changes in the transcript levels of the proapoptotic miRNA, mir-101, along with the relative changes in mRNA and protein levels of its target gene, $m c l-1$.

6.7 Summary figure of the relative changes in the transcript levels of the proapoptotic miRNAs, mir-15a and mir-16, along with the relative changes in mRNA and protein levels of their target gene, $b c l-2$.

6.8 Summary figure of the relative changes in the transcript levels of the proapoptotic miRNA, mir-101, along with the relative changes in mRNA and protein levels of its target gene, $m c l-1$.

1A Figure shows sample lanes from different proteins from the control condition such that each lane represents one gel probed with one target-specific antibody. 


\section{List of Tables}

\# Title

2.1 Summary of forward and reversed primers used in the study.

2.2 List of primary antibodies used with their dilution factor, expected molecular mass $(\mathrm{kDa})$ of the protein that the antibody detects, and the commercial source.

2.3 Probes used in the ELISA and EMSA procedures, ordered from Sigma Genosys. 32

2.4 Stem loop and forward microRNA primers that were used in the study. 


\section{Chapter 1:}

\section{General Introduction}




\section{Estivation and Metabolic Rate Depression}

Estivation is a state of aerobic dormancy used by animals in response to an arid environment and often triggered by limited water and food supplies. To escape the dry season, some animals burrow into the ground where they can remain immobile for periods of several months until the return of the wet season (Storey and Storey, 2010). To survive long periods of inactivity, adapted animals can strongly suppress their metabolic rate and enter a hypometabolic state (Storey and Storey, 2010; 2012). This state is characterized by (1) an overall suppression of metabolic rate, with estivators typically lowering metabolic rate to $20-30 \%$ percent of normal resting rate, (2) modification and reprioritization of many physiological and biochemical processes to conserve energy, and (3) the upregulation of specific pathways which play important roles in protecting and preserving the organism (Storey and Storey, 2010). To minimize energy consumption, macromolecule synthesis and degradation is limited during torpor, but expression of proteins with vital functions for survival, such as antioxidant defence and heat shock proteins is enhanced (Ramnanan et al., 2009; Malik and Storey, 2009).

Entry into torpor requires rapid, strong reversible controls to suppress global transcription and translation and quickly reorganize cellular pathways to enhance those with preservative properties. For example, reversible protein phosphorylation controls enzyme activity and the activation of key transcription factors that are responsible for the transcription of crucial stress-responsive genes (Storey and Storey, 2011). 


\section{Dehydration stress and Xenopus laevis}

A major priority during estivation becomes water conservation and the activation of a variety of cell preservation strategies required to protect the animal during long-term dormancy. Aestivators typically experience substantial dehydration stress and display high desiccation tolerance so studying the pathways activated by this stress are very important for understanding the phenomenon of estivation as a whole. This thesis attempts to further the current understanding of dehydration tolerance in a model anuran species that can estivate, the African clawed frog, Xenopus laevis (Storey and Storey, 2012).

This frog is found in sub-Saharan Africa where it lives in swamps, lakes, rivers and man-made wells. In laboratory science, $X$. laevis is as an important model in developmental biology and is of great interest for embryologists. Simple hormone manipulation can induce Xenopus to ovulate and mate any time of the year, which produces plenty of large and easily manipulated embryos. These embryos are used to study the function of various macromolecules, such as RNA and protein in living embryos (Chesneau et al., 2008).

$X$. laevis is almost exclusively an aquatic frog but has to face seasonal drying up of its ponds and survives by either (1) migrating to a distant body of water, or (2) by borrowing into the bottom mud and entering a state of estivation (Alexander and Bellerby, 1938). These frogs are very tolerant to dehydration and can withstand a severe loss of their total body water ( 30\%) (Romspert, 1975). It has been shown that Xenopus can survive a period of over eight months of estivation with no food or water (Tinsley 
and Kobel, 1996). Currently, little is known about the cellular processes which are activated and/or deactivated during the course of $X$. laevis estivation and dehydration.

\section{Antioxidant Defense}

Previous studies from our lab have established that protective antioxidant pathways are elevated in X. laevis tissues during dehydration (Malik and Storey, 2009; 2011). Antioxidant pathways are known to be an important part of hypometabolism in hibernating mammals as well as other species capable of entering torpor such as wood frogs (Hermes-Lima and Zenteno-Savin, 2002; Storey and Storey, 2010). It has been proposed that antioxidant pathways are activated under the stress condition as a means of preparing the cells for the time when dormancy is broken and rapid oxygen consumption is required for arousal. Another function of antioxidant pathway activation is to preserve cell viability for as long as possible by preventing oxidative damage to macromolecules during long-term dormancy (Storey and Storey, 2012). Recent studies with X. laevis show that antioxidant pathways may be upregulated in response to the stress of dehydration, as opposed to starvation or other triggers, during aestivation (Storey and Storey, 2012). Malik and Storey (2009) found that Nrf2, a transcription factor that responds to oxidative stress and enhances the transcription of antioxidant and detoxifying proteins, was elevated in tissues of Xenopus frogs that experienced a $28 \%$ dehydration of their total body water. Over 2-fold increases in Nrf2 were recorded in lung, heart, skin, liver and muscle. Antioxidant downstream targets of Nrf2 such as glutathione-Stransferase (GST) and aldo-keto reductase (AKR) were also elevated in all tissues studied compared to controls (Malik and Storey, 2009). 
FoxO1 is another transcription factor that plays a role in antioxidant defense during dehydration in Xenopus. Relative protein levels of FoxO1 increased significantly in liver of dehydrated frogs. Its downstream targets, superoxide dismutase (MnSOD) and catalase, which are both major antioxidant enzymes, were also significantly elevated in this tissue (Malik and Storey, 2011).

Both of these studies provide evidenced for the activation of antioxidant pathways in response to the stress of dehydration in X. laevis. It is likely that these mechanisms serve to protect the cells from oxidative damage during the estivation period and preserve the longevity of the stressed organs (Storey and Storey, 2012).

\section{Disuse atrophy}

Survival over long-term dormancy greatly depends on the organism's ability to maintain cellular integrity and organ function during the period of reduced metabolic rate. The torpor period is typically characterised by immobility and reduced organ activity, and as a result muscle atrophy becomes a great concern that needs to be addressed. When favourable conditions return, the animals must be able to rapidly return to their normal behaviour which depends on the ability to contract and use their skeletal muscles and move food through their digestive tract (Secor and Lignot, 2010). Skeletal muscle is a very pliable and responsive tissue that is able to undergo morphological changes that are moderated in response to activity. Serious muscle atrophy, characterised by a loss of whole muscle mass, muscle fibre cross sectional area and a reduced contractile force, has been recorded in humans after prolonged periods of muscle disuse due to bed rest, spaceflight, limb immobilization, etc. (Stein and Wade, 2005; Zhang et 
al., 2007). Studies using artificial limb immobilization as a model have shown that a period as short as several weeks can cause a severe reduction in muscle cross sectional area and decrease in contractile force in both mammals and amphibians (Hudson and Franklin, 2002).

Various species that undergo prolonged natural periods of estivation or hibernation have been studied as models for disuse atrophy to determine how to inhibit atrophy during prolonged periods of inactivity. Models have included hibernating bears, hamsters, and squirrels as well as estivating desert frogs (Hudson et al., 2005). For example, black bears (Ursus americanus) hibernate for 5 to 7 months, during which time they stay immobile in their cave without food or water. After 130 days of hibernation, researchers found a $23 \%$ loss in tibialis anterior muscle strength. Based on previous findings, the researchers predicted that an estimated $90 \%$ strength loss would have been seen in humans under similar conditions (Harlow et al., 2001). Ground squirrels were allowed to hibernate for several months before their soleus and EDL muscles were excised and compared to euthermic animals. A $15-20 \%$ decrease in skeletal muscle mass and fiber density was found in the hibernating squirrels compared to controls, a decrease that is considerably lower than found in non-hibernating mammalian models of muscle atrophy (Steffen et al., 1991).

Reducing muscle atrophy during dormancy is vital, especially in preparation for arousal, since muscle loss can threaten the reproductive success of animals (e.g. frogs and toads typically mate and lay eggs immediately upon arousal from seasonal estivation) or the ability to escape from predators (Hudson and Franklin, 2002). The first evidence for specific resistance to muscle atrophy during estivation was provided by analysing muscle 
mass and contractile power in green-striped burrowing frogs (Cyclorana alboguttata) following 3 months of estivation (Hudson and Franklin, 2002). Subsequent studies reported that after a period of prolonged estivation for 6-9 months C. alboguttata showed evidence of decreased muscle mass, muscle cross-sectional area (CSA) and myofiber CSA. Interestingly, all of the observed changes were seen in slow-twitch, oxidative muscles such as the illiobularis and satorius, which are non-jumping muscles. Indeed, smaller muscles may be metabolised as a last resort energy source to maintain aerobic metabolism through the estivation period (Mentle et al., 2009). The gastrocnemius muscle, which is the large muscle used for jumping and is essential for C. alboguttata) locomotion did not show atrophy after 9 months of estivation.

$X$. laevis is a non-selective carnivore that consumes both living and dead organisms found in its environment including arthropods, other amphibians, fish, and bird remains (Avila and Frye, 1977). During estivation it endures long term starvation while buried in the drying mud of a pond/stream bottom. To decrease their metabolic demands and conserve energy until favourable conditions return, adapted animals enter a state of hypometabolism and maintain aerobic metabolism by using their lipid reserves as the main fuel source. When Xenopus frogs enter dormancy, they switch to a primary dependence on breaking down lipid reserves from their fat bodies and liver stores to fuel energy metabolism. Tissues requiring glucose fuel (e.g. brain) may continue to receive glucose released from liver glycogen stores or from gluconeogenic reactions (probably from low rates of protein catabolism) or tissue may switch to using ketone bodies produced during lipid catabolism (as is well known to occur in mammalian hibernation). Following 4-6 months of starvation, when lipid stores have been largely depleted, 
Xenopus switch to catabolising protein as a main energy source (Mekle and Hanke, 1988). Evidence presented in a recent study by Mentle et al. (2009) corresponds to the finding that Xenopus switches to a protein based diet after several months of estivation, as they determined that the total muscle mass of small non-jumping muscles decreased following 6 months of $C$. alboguttata estivation. The authors propose that some muscle loss after 6 months of estivation occurs due to the depletion of lipid reserves and a switch to protein catabolism as opposed to atrophy due to immobility (Mentle et al., 2009).

A decrease in total muscle tissue mass appears to be an inevitable part of long term dormancy whether it is due to apoptotic signals associated with disuse atrophy or a switch to the catabolism of muscle proteins as an oxidative fuel source. However, disuse atrophy is not limited to skeletal muscle. It can also affect organs that are associated with digestion such as the liver, pancreas, stomach, and intestine. After 4 days of starvation, rat liver wet weight has been shown to decrease by as much as 50\% (Tessitore et al., 1999). Another study reported that after less than 10 days of starvation, hypatocyte volume decreased to $47 \%$ of normal in starved rats compared to controls (Pfeifer, 1972). The lack of food decreases the normal synthetic activities of the liver, which induces a decline in cell proliferation and an increase in apoptotic bodies and cell death (Tessitore et al., 1999). Lack of food during estivation seems to have a lesser effect in anuran species. Cramp et al. (2005) investigated the effect of long term estivation on the morphology of the stomach, liver, and small and large intestines to analyse whether mass was affected through the estivation period. The total mass of the stomach, small, and large intestines declined following 3 and 9 months of estivation while the total mass of the liver remained intact even after a 9 month term of dormancy. There was some 
evidence to indicate that apoptotic signals are at least in part responsible for digestive tissue atrophy such as the appearance of nuclear pyncosis in enterocytes of the small intestine (Cramp et al., 2005).

\section{Apoptosis}

Apoptosis, also known as programmed cell death, is a natural process of preserving tissue homeostasis through the maintenance of a consistent cell number and counterbalancing proliferation (Dupont- Versteegden, 2005). Other extrinsic and intrinsic signals can trigger the onset of the apoptotic cascade characterized by chromatin condensation, DNA fragmentation, breakdown of the nuclear envelope, and the proteolytic cleavage of structural polypeptides such as fodrin, which causes blebbing of the plasma membrane (Earnshaw et al., 1999). The cell disintegrates into apoptotic bodies and the remaining cellular components are taken up by adjacent cells through phagocytosis (Zimmermann and Green, 2001).

The aspartate-specific cysteine proteases (caspases) are a family of enzymes that are responsible for the execution of the cell death program through either an extrinsic or intrinsic pathway. These proteins are constitutively expressed in the cytoplasm in their inactive form and must be cleaved to their active form to be functional (Zimmermann and Green, 2001). Once activated, these enzymes catalyze the cleavage and degradation of several intracellular polypeptides that are important to the structural integrity of the cell and components of the DNA repair machinery (Earnshaw et al., 1999). The most abundant caspase in the cell is caspase- 3 and it is considered to be the "executioner" caspase together with caspase- 6 and -7 since these three enzymes coordinate the events 
associated with cell death. Other caspases, including -8 and -9 , are called the "initiator" caspases since they operate upstream of the executioner caspases by cleaving them to their active forms (Zimmermann and Green, 2001).

The extrinsic pathway of apoptosis begins with ligand binding to one of the tumor necrosis receptor (TNFR) family members such as binding of TNF- $\alpha$ to TNFR1. Once the death receptor is activated it recruits precursor molecules such as pro-caspase-8, the accumulation of which allows the molecules to process each other to mature, active caspase-8 enzymes (Zimmermann and Green, 2001). The "initiator" caspase-8 then cleaves caspase $-3,-6$ and -7 to their active forms and unleashes their apoptotic potential (Dupont-Versteegden, 2005). Caspases cleave vital polypeptides such as key structural components of the cytoskeleton (ex. fodrin and Gas-2) and nucleus (ex. Lamin A), proteins involved in DNA metabolism and repair (ex. topoisomerase I), proteins involved in cell cycle regulation (ex. p21) and other important proteins (Earnshaw et al., 1999).

The intrinsic pathway of apoptosis also known as the mitochondrial pathway is initiated by internal stimuli such as reactive oxygen species or other pro-apoptotic stimuli. Upon stimulation, the mitochondria release cytochrome $\mathrm{c}$ which joins the apoptotic protease activating factor-1 (Apaf-1), procaspase-9, and dATP to form an apoptosome. This complex cleaves procaspase- 3 to its active form thereby initiating the degradation of vital proteins and launching the apoptotic cascade (Figure 4.1) (Zimmermann and Green, 2001).

In addition to cytochrome c, over 30 other proteins are released from the mitochondria in response to a death signal. Two of these are Endonuclease $G$ and apoptosis inducing factor (AIF), which travel to the nucleus upon release from the 
mitochondria and assist in the degradation of DNA. Other proteins that are released from the mitochondria serve functions in the inactivation of inhibitors of apoptosis (IAPs) or control important steps in the activation of the Apaf-1/caspase-9 pathway (Borner, 2003).

The cell death program is carried out by a network of related and interconnected pathways each triggered by distinct stimuli and involving different key players, each opposed by several apoptotic inhibitors. Such inhibitors include (1) members of the Bcl-2 family; such as Bcl-2 and Bcl-xL, which are localized to the mitochondrial membrane where they work to prevent the release of cytochrome $\mathrm{c}$, and (2) inhibitors of apoptosis (IAPs) which can directly bind caspases.

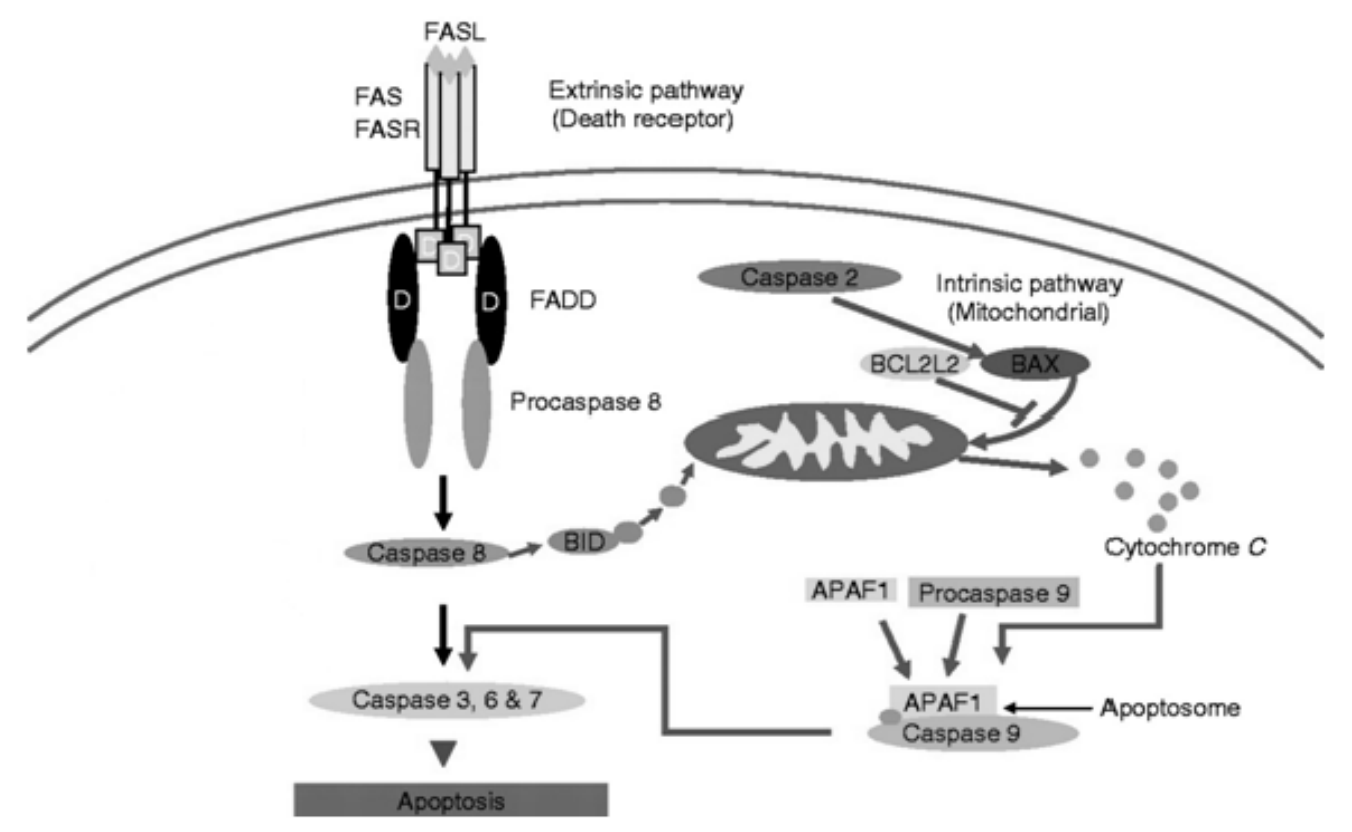

Figure 1.1: The intrinsic and extrinsic apoptotic pathway. The extrinsic pathway begins with a ligand binding to a death receptor (ex: FASL), which recruits adaptor molecules to activate caspase- 8 . The initiator caspcase- 8 activates the executer caspases $3,6,-7$. The intrinsic pathway begins when in response to a death stimulus, the mitochondria releases cytochrome $\mathrm{c}$ which joins with apoptotic protease activating factor1 (Apaf-1) and dATP to form an apoptosome. The complex activates caspase-9 which then cleaves caspase-3 to its active form and initiates the apoptotic cascade (figure adapted from Ruwanpura et al. 2010). 


\section{Apoptosis in Atrophy}

Apoptotic pathways appear to play a major role in skeletal muscle atrophy. Several studies have found that caspase- 3 abundance and activity increased in models of muscle atrophy such as hind-limb suspension (Yasuhara et al., 2000). Furthermore, the active forms of "executer" caspase-7 and "initiator" caspases-8, and -9 were elevated in models of muscle atrophy compared to controls (Tews et al., 2005). Caspase-3 is considered a key executor responsible for apoptosis in many cell types, however it has other destructive functions such as reducing muscle contractile power by the degradation of actin and myosin complexes (Du et al., 2004).

Further research is required to study the role of apoptotic cascades in muscle atrophy for different atrophy models such as denervation, hind-limb suspension, and old age, among others. It is clear that several apoptotic pathways are involved in the process of muscle atrophy, however details of the type of stimuli activating each pathway and the regulatory mechanisms involved are still to be investigated. For example, there is evidence of Bcl-2 involvement in muscle atrophy with one study showing an increase in the anti-apoptotic protein in patients with motor neuron disorders and another study showing a decrease in the protein in response to plexus injury (Jin et al., 2001; Tews et al., 1997). The results of these two studies, although contradicting, demonstrate that Bcl2 is an important player in muscle atrophy pathways. Both outcomes of Bcl-2 abundance in the cytoplasm seem logical, on the one hand, relative levels of Bcl-2 in the cytoplasm may decrease as a result of the onset of the cell death program and, on the other hand, this protein may accumulate to offset the apoptotic signals. Further studies are required to analyze the response of anti-apoptotic proteins during muscle atrophy. 
Medical studies are currently focused on the question of whether or not the inhibition of apoptotic pathways would attenuate muscle atrophy. For example exogenously administered XIAP, a member of the IAP family that can inhibit caspase-3, -7 and -9, was recently shown to inhibit muscle wasting in insulin-deficient mice (Wang et al., 2007). Such studies further the current knowledge of the role of apoptosis in muscle wasting and provide possible solutions for affected patients.

\section{Apoptosis in X. laevis}

X. laevis spends the dry season immobile, estivating in dry soil. During estivation, the animals' physiological and biochemical mechanisms undergo major reorganization done all with the single purpose of survival until favorable conditions return (Storey and Storey, 2010, 2012). Due to decreased activity, the preservation of cells and organs becomes a major concern during this period as it does in other species that are able to enter a state of dormancy (Story and Storey, 2012). Evidence from other amphibian species indicates that the rates of atrophy are much lower in the organs of estivating species, including skeletal muscle and digestive organs, than would be expected in nonestivators under similar conditions.

Limited studies have addressed the question of what biochemical pathways are activated during estivation that preserve tissue integrity and allow the animal to rapidly resume all of its normal activities when favorable conditions return. Nickolas et al. (2005) investigated the role of antioxidants in the context of muscular atrophy in another estivating frog species, C. albogutta. They found that total antioxidant activity remained unchanged in the estivating frogs compared to controls however as oxygen availability is 
limited in the burrow the abundance of anti-oxidant relative to oxygen uptake is in fact three times greater under dormancy conditions. Levels of the antioxidant proteins, catalase and glutathione peroxidase, were also unchanged between the two conditions (Nickolas et al., 2005). However, in dehydration studies of Xenopus, GST and AKR were significantly elevated in the lung, heart, skin, liver and muscle whereas MnSOD and catalase were found to be elevated only in dehydrated liver tissue (Malik and Storey, $2009 ; 2011)$. These mechanisms are put in place to protect the cells from reactive oxygen species, which may also play a role in inducing the cell death program.

There is some evidence to indicate that antioxidant pathways may serve an important role in protecting cells during the long period of estivation in amphibians. Surprisingly, no attention has been paid to the regulation of anti-apoptotic pathways in these estivating models. This thesis investigates the expression profile of two important families of transcription factors, Nuclear Factor $-\kappa \mathrm{B}(\mathrm{NF}-\kappa \mathrm{B})$ and Signal Transducers and Activators of Transcription (STAT), whose target genes include those with anti-apoptotic functions.

\section{Objectives and hypotheses}

My objectives are to evaluate the involvement of anti-apoptotic mechanisms in preserving tissues and limiting atrophy in $X$. laevis under stress conditions. The particular focus is on anti-apoptosis involvement in the response to dehydration stress, dehydration being an integral part of estivation. Hence, differential expression of anti-apoptotic mechanisms in response to dehydration would reasonably predict a similar response during estivation in nature. Studies focus on two major organs, skeletal muscle and liver, and are targeted at two levels of regulatory control of gene expression: transcription 
factor control of gene transcription and posttranscriptional control over mRNA translation by microRNAs.

Hypothesis 1: Anti-apoptotic mechanisms are activated in Xenopus laevis tissues as defense mechanism that preserves vital organs during dehydration and aestivation. Specifically, the transcription factors families, NF- $\mathrm{BB}$ and STAT, that are known to be involved in anti-apoptotic actions in other systems are activated in response to dehydration stress.

Hypothesis 2: MicroRNAs that are known to have anti-apoptotic functions are elevated in X. laevis organs in response to stress.

$N F-\kappa B$

NF- $\mathrm{kB}$, most commonly consisting of the subunits $\mathrm{p}-50$ and $\mathrm{p}-65$, is an important transcription factor first recognized for its involvement in the responses by cells to a variety of antigens such as bacteria and viruses (Pahl, 1999). It was later found to serve important functions by regulating key players in different cell processes such as cell survival (Bcl-xL), proliferation (cyclin D1), and angiogenesis (VEGF), among others (Nishikori, 2005). The anti-apoptotic targets of NF-kB include members of the bcl-2 family of proteins (e.g. bcl-2 and Bcl-xL), and the IAP family (e.g. c-IAP1, c-IAP2, XIAP) (Pahl, 1999; Nishikori, 2005). Chapter 3 evaluates the responses of the NF-KB pathway in liver and skeletal muscle of $X$. laevis, comparing control versus dehydrated frogs. The chapter focuses on the expression levels of the p-50 and p-65 subunits, the NF$\kappa \mathrm{B}$ inhibitor, IкB $\alpha$, as well as two downstream targets, $\mathrm{Bcl}-\mathrm{xL}$ and c-IAP1. 


\section{STATS}

The STAT family of proteins includes STAT1, STAT2, STAT3, STAT4, STAT5a, STAT5b, and STAT6. These transcription factors serve important roles in the protection against viral agents (ex. OAS and Mx), T helper cell differentiation (ex. ERM and IRF-1), erythropoiesis (ex. EPO) and, most importantly for the purposes of this thesis, cell growth and survival (Ihle, 2001). STAT3 downstream targets include members of the bcl-2 family (e.g. bcl-2, Bcl-xL, and mcl-1), and members of the IAP family (e.g. c-IAP1/2 and survivin) (Alvarez and Frank, 2004; Lanuti et al., 2009). STAT5 shares some of these downstream targets as it activates bcl-2, Bcl-xL, mcl-1 as well as a member of the IAP family, XIAP (Alvarez and Frank, 2004; Yu and Jove, 2004; Mohapatra et al., 2003). Chapter 4 focuses on the expression of the anti-apoptotic STAT proteins, STAT3 and STAT5, in the liver and skeletal muscle of $X$. laevis in response to dehydration and evaluates the expression of the downstream targets bcl-2 and mcl-1.

\section{$\operatorname{MicroRNA}$}

MicroRNAs (miRNAs) are recently discovered, small (18-24 nucleotides in length), non-coding RNA transcripts that are well conserved across many species including plants and viruses (Hwang and Mendell, 2006). These transcripts exert their regulatory role at the post-transcriptional level where they bind to protein-coding mRNA resulting in either translational repression or degradation of the target mRNA (Subramanian and Steer, 2010). A single miRNA has the power to regulate multiple mRNA transcripts while each mRNA can be regulated by a number of different miRNAs, creating a larger network of interrelated pathways (Jevanovic and Hengartner, 2006). 
This makes it complicated to draw conclusions regarding the function of a single miRNA type but many studies in the field of cancer research have pinpointed certain miRNAs as regulators of apoptosis. To date more than 30 miRNAs have been implicated in the regulation of apoptosis and as important markers of cancer (Subramanian and Steer, 2010). Chapter 5 focuses on the expression of both pro-apoptotic (mir-15a, mir-16 and mir-101) and anti-apoptotic (mir-19b, mir-21 mir-92a, mir-133, mir-145, mir-155, and mir-206) miRNA species that have been identified as major players in the cell death program in recent literature. 


\section{References}

Alexander S.S. and C.W. Bellerby. 1938. Experimental studies on the sexual cycle of the South African clawed toad (Xenopus laevis). J. Exp. Biol. 15: 74-81.

Alvarez, J.V. and D.A. Frank. 2004. Genome-wide analysis of STAT target genes: elucidating the mechanism of STAT-mediated oncogenesis. Cancer Biol Ther. 3(11): 1045-50.

Avila, V.L., and P.G. Frye. 1978. Feeding behavior of the African clawed Frog (Xenopus laevis Daudin): (Amphibia, Anura, Pipidae): effect of prey type. J. Herpetol. 12(3): 391396.

Borner, C. 2003. The Bcl-2 protein family: sensors and checkpoints for life-or-death decisions. Mol. Immunol. 39(11): 615-47.

Chesneau, A. Sachs, L.M., Chai, N., Chen, Y., Du Pasquier, L. Loeber, J., Pollet, N., Reilly, M., Weeks, D.L and O.J Bronchain. 2008. Transgenesis procedures in Xenopus. Biol Cell. 100(9): 503-21.

Cramp, R.L., Franklin, C.E. and E.A. Meyer. 2005. The impact of prolonged fasting during aestivation on the structure of the small intestine in the green-striped burrowing frog, Cyclorana alboguttata. Acta Zoologica. 86(1): 13-24.

Du, J., Wang, X., Miereles, C., Bailey, J.L., Debigare, R., Zheng, B., Price, S.R., and W.E. Mitch. 2004. Activation of caspase-3 is an initial step triggering accelerated muscle proteolysis in catabolic conditions. J. Clin. Invest. 113: 115-123.

Dupont-Versteegden, E.E. 2005. Apoptosis in muscle atrophy: relevance to sarcopenia. Exp. Gerontol. 40(6): 473-81.

Earnshaw, W.C., Martins, L.M. and S.H. Kaufmann. 1999. Mammalian caspases: structure, activation, substrates, and functions during apoptosis. Annu. Rev. Biochem. 68: $383-424$.

Harlow, H.J. Lohuis, T., Beck, T.D. and P.A Iaizzo. 2001. Muscle strength in overwintering bears. Nature. 409 (6823): 997.

Hermes-Lima, M. and T. Zenteno-Savin. 2002. Animal response to drastic changes in oxygen availability and physiological oxidative stress. Comp. Biochem. Physiol. C Toxicol. Pharmacol. 133(4): 537-56.

Hudson, N.J. and C.E Franklin. 2002. Effect of aestivation on muscle characteristics and locomotor performance in the green-striped burrowing frog, Cyclorana alboguttata. J. Comp. Physiol. B. 172(2): 177-82. 
Hudson, N.J., Lehnert, S.A., Ingham, A.B., Symonds, B., Franklin, C.E. and G.S. Harper. 2005. Lessons from an estivating frog: sparing muscle protein despite starvation and disuse. Am. J. Physiol. Regul. Integr. Comp. Physiol. 290(3): R836-43.

Hwang, H.W. and J.T. Mendell. 2006. MicroRNAs in cell proliferation, cell death, and tumorigenesis. Br J Cancer. 94(6):776-80.

Ihle, J.N. 2001. The stat family in cytokine signaling. Curr Opin Cell Biol. 13(2): 211117.

Jin, H., Wu. Z., Tian, T. and Y. Gu. 2001. Apoptosis in atrophic skeletal muscle induced by brachial plexus injury in rats. J. Trauma. 50: 31-35.

Lanuti, P., Bertagnolo, V., Pierdomenico, L., Bascelli, A. Santavenere, E., Alinari, L., Capitani, S., Miscia, S. and M. Marchisio. 2009. Enhancement of TRAIL cytotoxicity by AG-490 in human ALL cells is characterized by downregulation of cIAP-1 and cIAP-2 through inhibition of Jak2/Stat3. Cell Research. 19:1079-1089.

Malik, A.I. and K. B. Storey. 2009. Activation of antioxidant defense during dehydration stress in the African clawed frog. Gene. 442: 99-107.

Malik, A.I. and K. B. Storey. 2011.Transcriptional regulation of antioxidant enzymes by FoxO1 under dehydration stress. Gene. 485: 114-119.

Mentle, B.L. Hudson, N.J., Harper, G.S., Cramp, R.L. and C.E. Franklin. 2009. Skeletal muscle atrophy occurs slowly and selectively during prolonged aestivation in Cyclorana alboguttata (Günther 1867). J. Exp. Biol. 212(22): 3664-3672.

Mekle, S. and W. Hanke. 1988. Long-term starvation in Xenopus laevis (Daudin) - I. Effects on general metabolism. Comp. Biochem. Physiol. 89(4): 719-730.

Mohapatra, S. Chu, B., Wei, S., Djeu, J., Epling-Burnette, P.K., Loughran, T., Jove, R. and W.J. Pledger. 2003. Roscovitine inhibits STAT5 activity and induces apoptosis in the human leukemia virus type 1-transformed cell line MT-2. Cancer Res. 63(23): 8523-8530

Nishikori, M. 2005. Classical and alternative NF-kB activation pathways and their roles in lymphoid malignancies. J. Clin. Exp. Hematopathol. 45(1): 15-24.

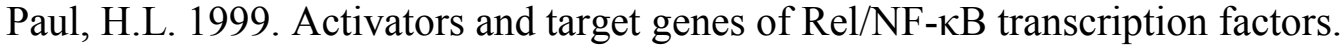
Oncogene 18: 6853- 6866.

Pfeifer, U. 1972. Cellular autophagy and cell atrophy in the rat liver during long-term starvation. Virchows Archiv B. 12(1): 195-211 
Ramnanan, C.J., Allan, M.E., Groom, A.G. and K.B. Storey. 2009. Regulation of global protein translation and protein degradation in aerobic dormancy. Mol. Cell. Biochem. 323: 9-20.

Romspert, A.P. 1975. Osmoregulation of the African clawed frog, Xenopus laevis, in hypersaline media. Comp. Biochem. Physiol. A. 54: 207-210.

Sector, S.M. and J.H. Lingot. 2010. Morphological plasticity of vertebrate aestivation. Prog. Mol. Subcell. Biol. 49: 183-208.

Steffen, J.M., Koebel,, D.A., Musacchia, X.J. and W.K Milson. 1991. Morphometric and metabolic indices of disuse in muscles of hibernating ground squirrels. Comp Biochem Physiol B. 99(4): 815-819.

Stein, T.P and C.E. Wade. 2005. Metabolic consequences of muscle disuse atrophy. J. Nutr. 135(7): 1824S-1828S.

Storey, K.B. and J.M. Storey. 2010. Metabolic regulation and gene expression during aestivation. In: Aestivation: Molecular and Physiological Aspects (Navas, C.A. and Carvalho, J.E., eds), Progress in Molecular and Subcellular Biology, Springer, Heidelberg, Vol. 49, pp. 25-45.

Storey, K.B. and J.M. Storey. 2011. Strategies of molecular adaptation to climate change: the challenges for amphibians and reptiles. In: Temperature Adaptation in a Changing Climate (Storey, K.B. and Tannino, K., eds), CABI Publishers: Wallingford, UK.

Storey K.B and J.M Storey. 2012. Aestivation: signaling and hypometabolism. J. Exp. Biol. 215(Pt 9): 1425-33.

Subramanian, S. and C.J. Steer.2010. MicroRNAs as gatekeepers of apoptosis. J Cell Physiol. 223(2):289-98.

Tessitore, L., Tomasi, C. and M. Greco. 1999. Fasting-induced apoptosis in rat liver is blocked by cycloheximide. Eur. J. Cell Biol. 78(8): 573-9.

Tews, D.S., Behrhof, W., and S. Schindler. 2005. Expression patterns of initiator and effector caspases in denervated human skeletal muscle. Muscle Nerve. 31: 175-181.

Tinsley, R.C. and H.R. Kobel. 1996. The Biology of Xenopus. Oxford Scientific Press, Oxford.

Vander-Heiden, M.G. and C.B. Thompson. 1999. Bcl-2 proteins: regulators of apoptosis or of mitochondrial homeostasis? Nat Cell Biol. 1: E209-E214.

Wang, X.H., Hu, J., Du. J., and J.D. Klein. 2007. X-chromosome linked inhibitor of apoptosis protein inhibits muscle proteolysis in insulin-deficient mice. Gene Ther. 14: 
711-20.

Yasuhara, S., Perez M.E., Kanakubo, E., Yasuhara, Y., Shin, Y.S., Kaneki, M., Fujita, T. and J.A. Martyn. 2000. Skeletal muscle apoptosis after burns is associated with activation of proapoptotic signals. Am. J. Physiol. Endocrinol. Metab. 279: E1114-E1121.

$\mathrm{Yu}, \mathrm{H}$. and R. Jove. 2004. The STATs of cancer- new molecular targets come of age. Nat Rev Cancer. 4(2): 97-105.

Zhang, P. Chen, X., and M. Fan. 2007. Signaling mechanisms involved in disuse muscle atrophy. Med Hypotheses. 69(2): 310-21.

Zimmermann, K.C. and D.R. Green. 2001. How cells die: apoptosis pathways. J. Allergy Clin. Immunol. 108(4): S99-103. 


\section{Chapter 2:}

\section{General Materials and Methods}




\section{Animal care and treatment}

X. laevis frogs were obtained from the Department of Zoology, University of Toronto and held in tanks of dechloraminated water at $22^{\circ} \mathrm{C}$. To begin the dehydration experiments the animals were divided into three groups: (1) control, (2) experimental, medium dehydration, and (3) experimental, high dehydration groups. The experimental dehydration groups were placed in closed containers. The percentage dehydration was determined by periodically taking out the animals from the tank and quickly weighing them. The animals were monitored in this fashion over 3-5 days. The change in mass was used to calculate the percentage of total body water lost via the following equation:

$$
\% \text { water lost }=[(\mathrm{Wi}-\mathrm{Wd}) /(\mathrm{Wi} * \mathrm{BWCi})] * 100
$$

Where Wi is the initial mass of an individual, Wd is the mass during experimental dehydration, and BWCi is the initial body water content of frogs before dehydration. BWCi was determined from measurements of the wet and dry masses of 7 euthanized frogs; the value was $0.741 \pm 0.019 \mathrm{~g}$ water per $\mathrm{g}$ body mass. The medium dehydration and high dehydration groups of frogs had reached a mean water loss of $16.44 \pm 0.33 \%$ and $31.18 \pm 0.83 \%$, respectively. Frogs were quickly pithed and tissues were quickly dissected and stored at $-80^{\circ} \mathrm{C}$.

\section{Total RNA isolation and quality assessment}

Four samples of $X$. laevis skeletal muscle and liver from control and both experimental groups were removed from $-80^{\circ}$ storage and placed in liquid nitrogen. All 
the pipette tips used in the procedure were treated with $0.1 \% \mathrm{v} / \mathrm{v}$ diethylpyrocarbonate (DEPC) and autoclaved. Samples of frozen skeletal muscle and liver ( $\sim 50 \mathrm{mg}$ each) were quickly weighed and homogenizd in $1 \mathrm{~mL} \mathrm{Trizol}^{\mathrm{TM}}$ reagent (Tri-Reagent, Invitogen) using a Polytron PT10 homogenizer. Subsequently, $200 \mu$ l of chloroform was added to each sample. The samples were then centrifuged at $10,000 \mathrm{x}$ g for 15 minutes at $4{ }^{\circ} \mathrm{C}$ in the Eppendorf centrifuge (5810R). The centrifugation separates the samples into an organic layer (containing organelles and lipid fractions), a DNA layer at the interface, and an upper aqueous layer containing RNA. This top layer was removed and placed into separate, clean, sterile microcentrifuge tubes. To each sample, $500 \mu 1$ of isopropanol was added and briefly vortexed. The RNA was allowed to precipitate at room temperature for 10 minutes followed by centrifugation at $12,000 \mathrm{rpm}$ for 15 minutes. The supernatant was removed and the RNA pellet was washed with $1 \mathrm{ml}$ of $70 \%$ ethanol. The tubes were spun again at $7500 \mathrm{rpm}$ for 5 minutes. The supernatant was removed and the tubes were allowed to dry for 10 minutes. The pellet was resuspended in $50 \mu 1$ of DEPC treated ddH20. In total 12 RNA samples were prepared for each tissue; 4 control, 4 medium dehydration and 4 high dehydration. The $260 / 280 \mathrm{~nm}$ ratio was used to assess the RNA purity and concentration on a GeneQuant Pro spectrophotometer (Pharmacia). Only RNA with $260 / 280 \mathrm{~nm}$ ratio of greater than 1.8 were used in this study. To ensure proper RNA isolation the samples were run on a 1\% agarose gel stained with $2 \mathrm{X}$ Sybr Green I (Invitrogen) using a horizontal electrophoresis apparatus. Two sharp bands were visible, representing the $28 \mathrm{~S}$ and $18 \mathrm{~S}$ ribosomal RNA, when viewed under UV light using a ChemiGenius imaging system (Syngene, Frederick, MD, USA). All RNA samples were subsequently diluted to $1 \mu \mathrm{g} / \mu 1 \mathrm{RNA}$ using DEPC-treated $\mathrm{ddH}_{2} \mathrm{O}$. 


\section{Primer Design}

Nucleotide sequences for all genes under study were obtained using the NCBI whole genome shotgun BLAST bioinformatics program (http://www.ncbi.nlm.nih.gov/). A list of possible primers was generated using the Primer Designer program, v3.0 (Scientific and Educational Software). Suitable primers were then selected on a number of different parameters obtained from IDT OligoAnalyzer 3.1 program (http://www.id tdna.com/analyzer/ Applications/OligoAnalyzer/). Primers were selected based on the following parameters: size (20 nt), containing 40-60\% GC content, having minimum sequence repeats and primer annealing temperature between $55^{\circ} \mathrm{C}$ and $65^{\circ} \mathrm{C}$. Finally, the forward and reverse primers were selected with similar melting temperatures $\left( \pm 1^{\circ}\right)$. Selected primer sets were chosen and ordered from Sigma Genosys. A summary of the primers used can be found in Table 2.1.

Table 2.1: Summary of forward and reversed primers used in the study.

\begin{tabular}{|l|l|l|}
\hline Gene & Forward Primer $\left(\mathbf{5}^{\prime} \rightarrow \mathbf{3}\right.$ ') & Reverse Primer $\left(\mathbf{5}^{\prime} \rightarrow \mathbf{3}\right.$ ') \\
\hline$\alpha$-tubulin & AAGGAAGATGCTGCCAATAA & GGTCACATTTCACCATCTG \\
\hline Bcl-XL & AGATATCAGCGTGCCTTCAG & GTCTGTCCATGATAGGCGAC \\
\hline c-IAP & TGTGAACTCTATCGGCTCTC & GTGCTCGGACATGGCATTGT \\
\hline P53 & CCGTCACTTCATGTGCTGTC & ACTTGCGGCACTTAATGGTC \\
\hline Bcl-2 & ATCCAGATGCCAGTCGTGCT & ACAATCTCCACGCACATGAC \\
\hline mcl-2 & CTTCCTGTCTCCTCCAACAT & GAACTGCGTGAAGTGCTCTG \\
& & \\
\hline
\end{tabular}




\section{Complementary DNA (cDNA) synthesis}

A $5 \mu$ g aliquot (in $5 \mu \mathrm{l}$ ) of RNA from each sample was diluted with $5 \mu$ l of DEPC treated dd $\mathrm{H}_{2} \mathrm{O}$. Briefly, $1 \mu \mathrm{l}$ of oligo-dT primer $\left(200 \mathrm{ng} / \mu \mathrm{l}, 5^{\prime}-\right.$ TTTTTTTTTTTTTTTTTTTTTTV-3'; V= A, G, or C) was added to each PCR tube. The samples were incubated in a thermal cycler (Mastercycle, Eppendorf) for 5 minutes at $65^{\circ} \mathrm{C}$ to promote annealing of the oligo-dT to the mRNA polyA tail. The mixture was then chilled rapidly on ice and $4 \mu 1$ of $5 \mathrm{X}$ first strand buffer, $2 \mu 1$ of $0.1 \mathrm{M} \mathrm{DTT}, 1 \mu 1$ of $10 \mathrm{mM}$ dNTPs (BioShop) and $1 \mu \mathrm{l}$ of M-MLV reverse transcriptase (Invitrogen) were added to each tube for a total of $19 \mu 1$. The reactions were placed back into the thermal

cycler and incubated for 45 minutes at $42^{\circ} \mathrm{C}$. The cDNA was then diluted to $10^{-1}$ and $10^{-2}$ and placed in a $-20^{\circ} \mathrm{C}$ fridge until use.

\section{Reverse Transcriptase-PCR}

Reverse Transcriptase Polymerase Chain Reaction (RT-PCR) was used to amplify the genes under study from the cDNA samples. Each PCR reaction consisted of $15.25 \mu \mathrm{L}$ of DEPC treated water, $5 \mu \mathrm{L}$ of diluted cDNA, $1.25 \mu \mathrm{L}$ of primer mixture (to a final concentration of $1.5 \mu \mathrm{M}$ forward and $1.5 \mu \mathrm{M}$ reverse), $2.5 \mu \mathrm{L}$ of $10 \times$ PCR buffer (Invitrogen), $1.5 \mu \mathrm{L}$ of $50 \mathrm{mM} \mathrm{MgCl} 2,0.5 \mu \mathrm{L}$ of dNTP mixture (25 mM each) and $1 \mu \mathrm{L}$ of Taq Polymerase (Invitogen). Each sample had a total volume of $25 \mu 1$. Samples were tested at different temperatures to find the optimum primer annealing temperature.

The program for all PCR reactions was as follows; denaturation at $94^{\circ} \mathrm{C}$ for 7 min, followed by a experimentally determined number of cycles of $94^{\circ} \mathrm{C}$ for 1 minute, primer annealing at the determined optimum temperature for 1 minute, elongation at $72^{\circ} \mathrm{C}$ for 1.5 minutes. The final elongation was a single cycle $72^{\circ} \mathrm{C}$ for 10 minutes. The 
number of cycles depended on the gene of interest and cDNA dilution. PCR products were electrophoretically separated on a $200 \mathrm{~mL} 1 \%$ agarose gel. Loading dye $(2 \mu \mathrm{l})$ and Sybr-Green I ( $1 \mu$ l of 1:200 in TAE buffer; Invitrogen) were added to each sample. Each gel was made using $2 \mathrm{~g}$ of agarose (BioShop) and $200 \mathrm{~mL}$ of $1 \mathrm{X}$ TAE buffer made by adding $4 \mathrm{~mL}$ of 50X TAE stock (2 M Tris-HCl, $1 \mathrm{M}$ acetic acid, $0.05 \mathrm{M}$ EDTA, adjusted to $\mathrm{pH} 8.5$ ) to $196 \mathrm{~mL}$ of DEPC-treated water. A $14 \mu \mathrm{l}$ aliquot of each amplified PCR sample was loaded into the solidified gel and run at $130 \mathrm{~V}$ for 20 minutes. The bands were visualized under UV light using the ChemiGenius Bio Imaging System and the bands were quantified using the GeneTools software. Each band was quantified and standardized against bands for $\alpha$-tubulin amplified from the same cDNA sample. The cDNA bands were excised with a razor blade. Samples were isolated from gel fragments using a freeze-thaw cycle in liquid nitrogen, followed by a centrifugation at $7000 \mathrm{xg}$ for 7 minutes at room temperature. While undergoing centrifugation, samples were passed through a filter into a clean tube. Purified cDNA samples were sent for sequencing to BioBasic (Markham, Ontario). The sequences were verified as encoding the correct genes using the program BLASTN (http://www.ncbi.nlm.nih.gov/blast) at the NIH.

\section{Total protein extraction}

Xenopus liver and skeletal muscle tissue samples were removed from storage at $-80^{\circ} \mathrm{C}$ and placed in liquid nitrogen. Frozen liver samples were crushed under liquid nitrogen and then homogenized using a using a Polytron PT10. Each sample was suspended 1:2.5 w:v in homogenizing buffer (20 mM Hepes, $\mathrm{pH} 7.5,200 \mathrm{mM} \mathrm{NaCl}, 0.1$ mM EDTA, $10 \mathrm{mM} \mathrm{NaF}, 1 \mathrm{mM} \mathrm{Na} \mathrm{VO}_{4}, 10 \mathrm{mM} \beta$-glycerophosphate) with a few 
crystals of phenylmethylsulfonyl fluoride (PMSF; BioShop) and $1 \mu \mathrm{L}$ protease inhibitor cocktail added (Sigma-Aldrich, Oakville, ON). The samples were then centrifuged at $4^{\circ} \mathrm{C}$ for 15 minutes at 10,000 rpm. The supernatant was removed and placed in new clean tubes. Soluble protein concentration was determined using the BioRad protein assay using the BioRad reagent (BioRad Laboratories). A calculated amount of homogenizing buffer was then added to adjust the protein concentration to $10 \mu \mathrm{g} / \mu \mathrm{l}$. The samples were then mixed in a 1:1 ratio with 2 X SDS loading buffer (100 mM Tris-HCl, $4 \% \mathrm{w} / \mathrm{v}$ lauryl sulphate, $20 \% \mathrm{v} / \mathrm{v}$ glycerol, $0.2 \% \mathrm{w} / \mathrm{v}$ bromophenol blue, $10 \% \mathrm{v} / \mathrm{v} 2$-mercaptoethanol). The final product concentration was $5 \mu \mathrm{g} / \mu 1$. The proteins were denatured by placing the tubes in boiled water for 5 minutes. All the samples were stored in the $-80^{\circ} \mathrm{C}$ freezer until use.

\section{Nuclear protein extraction}

Samples of liver and muscle ( $\sim 0.6$ g each) were weighed and manually

pulverized in liquid nitrogen using a mortar and pestle, followed by the addition $100 \mu 1$ of homogenizing buffer (10 mM HEPES, pH 7.9, 10 mM KCl, 10 mM EDTA, 20 mM ßglycerophosphate, $10 \mu \mathrm{l}$ of $100 \mathrm{mM}$ DTT stock, and $10 \mu 1$ Protease Inhibitor Cocktail) and homogenization with a Dounce homogenizer. This procedure allows for the breakdown of the cell but leaves the nucleus intact. The samples were then centrifuged at $10,000 \mathrm{rpm}$ for $10 \mathrm{~min}$ at $4{ }^{\circ} \mathrm{C}$. The supernatant (containing the cytoplasmic extract) was discarded and the pellet of nuclei was resuspended in $90 \mu \mathrm{l}$ of extraction buffer $(10 \mathrm{mM}$ HEPES, pH 7.9, 0.2 M NaCl, 0.5 mM EDTA, 50\% v/v glycerol, $10 \mathrm{mM}$ ßglycerophosphate, $1.5 \mu \mathrm{l}$ of $100 \mathrm{mM}$ DTT and $1.5 \mu \mathrm{l}$ of Protease Inhibitor Cocktail). The 
sample tubes were placed on ice and left on a rocking platform for 1 hour, during which the high salt concentration of the extraction buffer causes the nuclei to lyse. The samples were then centrifuged at $10000 \mathrm{rpm}$ for $10 \mathrm{~min}$ at $4^{\circ} \mathrm{C}$, and the final supernatant, containing the nuclear extract, was removed. The BioRad assay was used to quantify the amount of protein in each nuclear sample and then all samples were standardized to the same concentration by the addition of small aliquots of homogenizing buffer; final concentrations were $4 \mu \mathrm{g} / \mu \mathrm{l}$ for skeletal muscle and $8 \mu \mathrm{g} / \mu \mathrm{l}$ for liver samples. Samples were stored at $-80^{\circ} \mathrm{C}$ until use.

To produce nuclear homogenates used for western blotting, half of the extract from each sample was transferred to a new tube and the samples were mixed in a 1:1 ratio with $2 X$ SDS loading buffer. The final product concentrations were $2 \mu \mathrm{g} / \mu \mathrm{l}$ for the skeletal muscle samples and $4 \mu \mathrm{g} / \mu \mathrm{l}$ for the liver samples. The proteins were denatured by placing the tubes in boiled water for 5 minutes. All the samples were stored at $-80^{\circ} \mathrm{C}$ until use.

\section{Western Blots}

Aliquots of samples containing $25 \mu \mathrm{g}$ protein were loaded into lanes on polyacrylamide gels, together with a lane containing prestained molecular weight standards (Froggabio) and separated using a discontinuous buffer system. Gels were 8$12 \%$ acrylamide depending on the molecular weight of the protein of interest and the stacking gel was 5\% in all cases. Composition of the stacking gel was: $130 \mu 11.0 \mathrm{M}$ Tris$\mathrm{HCl}, 170 \mu \mathrm{l} 30 \%$ bis-acrylamide, $680 \mu \mathrm{l}$ water, $10 \mu \mathrm{l}$ 10\% SDS, $10 \mu \mathrm{l} 10 \%$ APS, and 5 $\mu 1$ TEMED. Resolving gels of $8-12 \%$ were prepared by varying the volumes of bis- 
acrylamide and water; for example, the $10 \%$ resolving gel consisted of $1.3 \mathrm{ml} 1.5 \mathrm{M}$ Tris-

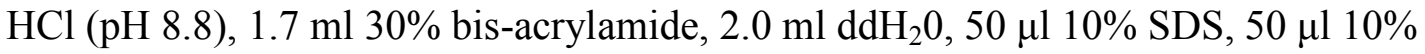
APS, $8 \mu 1$ TEMED.

Electrophoresis was carried out at $180 \mathrm{~V}$ for 1 hour using the BioRad MiniProtean 3 system using $1 \mathrm{X}$ Tris-glycine running buffer diluted from the $10 \mathrm{X}$ stock (84 $\mathrm{mM}$ Tris- $\mathrm{HCl}, 2.5 \mathrm{mM}$ glycine, $34.7 \mathrm{mM} \mathrm{SDS}$ in $\mathrm{ddH}_{2} \mathrm{O}$ ). Proteins on the gel were then electroblotted onto polyvinylidenedifluoride (PVDF) membrane (Immobilon-P Transfer Membrane, Millipore, Bedford, MA) using a BioRad mini Trans-Blot cell. The transfer buffer used was made using $60.6 \mathrm{~g}$ Tris- $\mathrm{HCl}$ and $288.0 \mathrm{~g}$ glycine added into $16 \mathrm{~L}$ water and $4 \mathrm{~L}$ methanol. The transfer was carried out at a constant current of $160 \mathrm{~mA}$ per transfer box for 1.5 hours in about $400-500 \mathrm{ml}$ of transfer buffer $(25 \mathrm{mM}$ Tris- $\mathrm{HCl} \mathrm{pH}$ $8.5,192 \mathrm{mM}$ glycine and 10\% v/v methanol). The membranes were placed into plastic boxes and washed in TBST $(10 \mathrm{mM}$ Tris- $\mathrm{HCl}, \mathrm{pH} 7.5,150 \mathrm{mM} \mathrm{NaCl}, 0.05 \% \mathrm{v} / \mathrm{v}$ Tween20) for 15 minutes. The membranes were blocked with 70-100 kDa polyvinyl alcohol (PVA, Sigma-Aldrich) at a concentration of $0.5 \mu \mathrm{g} / \mu \mathrm{l}$ for 45 seconds. After blocking, the membranes were probed with 1:1000 primary antibody for 24 hours in the $4^{\circ} \mathrm{C}$ fridge (Table 2.2). The membranes were washed once more with TBST at room temperature and probed with 1:2000 HRP-conjugated secondary antibody for 1 hour. Membranes were washed for 20 minutes in TBST at room temperature.

Immunoblots were developed using enhanced chemiluminescence $\left(\mathrm{H}_{2} \mathrm{O}_{2}\right.$ and Luminol) and visualized using a Chemi-Genius BioImaging system (Syngene, Frederick, MD). The GeneTools program was used to quantify the protein band densities. The presence of the appropriate band was confirmed by loading $3 \mu l$ of protein ladder to the 
gel (Froggabio). The membranes were subsequently stained with Coomassie blue $(0.25 \%$ w/v Commassie brilliant blue, $7.5 \% \mathrm{v} / \mathrm{v}$ acetic acid, $50 \%$ methanol) and densities of a group of bands that did not vary between samples were quantified and used to standardize the immunoblot band densities to control for minor variations in protein loading.

Table 2.2: List of primary antibodies used with their dilution factor, expected molecular mass $(\mathrm{kDa})$ of the protein that the antibody detects, and the commercial source.

\begin{tabular}{|l|l|l|}
\hline Antibody & $\begin{array}{l}\text { Molecular } \\
\text { Weight (kDa) }\end{array}$ & Company \\
\hline p50 Abs (Abs 337), pAb, Rabbit 1:1000 & $\sim 50$ & GenScript (\#A00470) \\
\hline p50 (Ser 337), Rabbit, 1:1000 & $\sim 50$ & GenScript (\#A00585) \\
\hline p65 Abs (Abs 529), Rabbit, 1:1000 & $\sim 65$ & GenScript (\#A00459) \\
\hline p65 (Ser 529), Rabbit, 1:1000 & $\sim 65$ & GenScript (\#A00330) \\
\hline IאB- $\alpha$ (Abs 32, 36), Rabbit, 1:1000 & $\sim 40$ & GenScript (\#A00609) \\
\hline IкB- $\alpha$ (Ser 32, 36), Rabbit, 1:1000 & $\sim 40$ & GenScript (\#A00359) \\
\hline Bcl-xL, Mouse, 1:1000 & $\sim 26$ & Santa Cruz (\#sc-8392) \\
\hline c-IAP1, Rabbit, 1:1000 & $\sim 70$ & Santa Cruz (\#sc-7943) \\
\hline P53, Rabbit, 1:1000 & $\sim 53$ & Cell signalling (\#2527) \\
\hline STAT 1, Rabbit, 1:1000 & $\sim 84,91$ & Cell signalling (\#9171) \\
\hline STAT 2, Rabbit, 1:1000 & $\sim 113$ & Cell signalling (\#4441) \\
\hline STAT 3 (Ser 727), Rabbit, 1:1000 & $\sim 79,86$ & Cell signalling (\#9145) \\
\hline STAT 3 (Tyr 705), Rabbit, 1:1000 & $\sim 86$ & Cell signalling (\#9134) \\
\hline STAT5 (Tyr 694), Rabbit, 1:1000 & $\sim 90$ & Cell signalling (\#9359) \\
\hline STAT 6, Rabbit, 1:1000 & $\sim 110$ & Cell signalling (\# 9351) \\
\hline Bcl-2, Rabbit, 1:1000 & $\sim 26$ & Cell signalling (\#2872) \\
\hline Bcl 2 (Thr 56), Rabbit, 1:1000 & $\sim 28$ & Cell signalling (\#2875) \\
\hline Bcl-2 (Ser 70), Rabbit, 1:1000 & Cell signalling (\#2827) \\
\hline Mcl-1, Rabbit, 1:1000 & $\sim 28$ & Cell signalling (\#5453) \\
\hline
\end{tabular}

\section{Enzyme linked immunosorbent assay (ELISA)}

The binding activity of p50, p65, STAT3 and STAT5 to a specific DNA sequence were assessed using biotin labelled probes. Biotin labelled probes were designed to contain the DNA-binding element of the transcription factors under investigation and 
ordered from Sigma Genosys. Summary of the used biotin labelled probes and their complements can be found in Table 2.3.

Table 2.3. Probes used in the ELISA and EMSA procedures, ordered from Sigma Genosys.

\begin{tabular}{|l|l|l|}
\hline Probe & Biotin labelled probe $\left(\mathbf{5}^{\prime} \rightarrow \mathbf{3}^{\prime}\right)$ & Reverse Complement $\left(\mathbf{5}^{\prime} \rightarrow \mathbf{3}^{\prime}\right)$ \\
\hline $\begin{array}{l}\text { NF- } \mathrm{kB} \\
(\mathrm{p} 50 / \mathrm{p} 65)\end{array}$ & $\begin{array}{l}\text { 7-CACAGTTGAGGGGACTTTCC } \\
\text { CAGGC }\end{array}$ & $\begin{array}{l}\text { GCCTGGGAAAGTCCCCTCA } \\
\text { ACTGTG }\end{array}$ \\
\hline STAT $(3 / 5)$ & $\begin{array}{l}\text { 7-GATCCTTCCCGGAACGTATTC } \\
\text { CGAGAATCT }\end{array}$ & $\begin{array}{l}\text { AGATTCTCGGAATACGTTCC } \\
\text { GGGAAGGATC }\end{array}$ \\
\hline
\end{tabular}

The prepared oligonucleotides were suspended in DEPC treated water at a concentration of 500 pmoles $/ \mu 1(500 \mu \mathrm{M})$. To prepare a double stranded probe, $10 \mu \mathrm{l}$ of the biotinylated oligonucleotide were combined with $10 \mu$ of the antisense oligonucleotide (final concentration $250 \mathrm{pmoles} / \mu \mathrm{l}$ ). The oligonucleotides were denatured by incubating at $94^{\circ} \mathrm{C}$ in the thermal cycler (Mastercycler, Eppendorf) for 10 minutes, followed by a 10 minute incubation at room temperature to allow the denatured oligonucleotides to anneal to each other. The double-stranded probes were stored at $-20^{\circ} \mathrm{C}$.

Double-stranded probe was diluted in PBS buffer $(137 \mathrm{mM} \mathrm{NaCl}, 2.7 \mathrm{mM} \mathrm{KCl}$, $10 \mathrm{mM} \mathrm{Na}_{2} \mathrm{HPO}_{4}$, and $2 \mathrm{mM} \mathrm{KH}_{2} \mathrm{PO}_{4}, \mathrm{pH} 7.4$ ) to $0.8 \mathrm{pmol} / \mu 1$. Each sample well on the plate was coated with $40 \mathrm{pmol}$ of probe $(50 \mu 1$ in total) and plate was incubated at room temperature for 1 hour. Following this, the plate was washed 3 times with $200 \mu 1 /$ well of wash buffer (PBS containing 0.1\% Tween 20) followed by one wash with PBS. The plate 
was inverted after each wash and tapped on a paper towel to get rid of excess wash buffer.

A master mix was prepared containing the TF-binding buffer ( $5 \mathrm{mM}$ HEPES, 25 $\mathrm{mM} \mathrm{KCl}, 0.25 \mathrm{mM}$ EDTA, $1.5 \mathrm{mM} \mathrm{MgCl}_{2}, 50 \% \mathrm{v} / \mathrm{v}$ glycerol, $2.5 \mathrm{mg} / \mathrm{ml} \mathrm{BSA}$, and 0.25\% NP-40, pH 7.9), $100 \mathrm{mM}$ DTT and salmon sperm DNA $(20 \mu \mathrm{g} / \mu \mathrm{l})$ in a 200:1:0.2 ratio, respectively. A volume the nuclear extract producing the desired protein concentration was added to the master mix and $50 \mu \mathrm{l}$ of this solution was added to all the wells except for the "no protein" control wells which received $50 \mu$ of the master mix without any added extract. The plate was incubated for $1 \mathrm{~h}$ at room temperature, shaking at $100 \mathrm{rpm}$. Following the incubation period, the plate was washed $3 \mathrm{X}$ with $200 \mu \mathrm{l}$ of washing buffer. Primary antibody, diluted 1:2000 v:v in PBST, was added at $60 \mu 1 /$ well and incubated for 1 hour. The plate was washed and then $60 \mu 1$ of secondary antibody (1:4000 v:v dilution) was added to each well and incubated for 1 hour. The wells were washed with PBS and then $60 \mu$ of tetramethylbenzidine (TMB, Bioshop) were added to each well. After sufficient colour development, $60 \mu 1$ of the stop solution $(1 \mathrm{M} \mathrm{HCl})$ were added to each well to terminate the reaction.

The plate was then scanned using a Multiskan spectrophotometer measuring absorbance at $450 \mathrm{~nm}$ with a reference wavelength of $655 \mathrm{~nm}$.

\section{Electrophoretic-Mobility Shift Assay (EMSA)}

An EMSA assay was carried out to validate the DNA binding results obtained using the ELISA protocol. Biotin labeled probes containing the DNA binding element were designed and obtained from Sigma-Genosys. Double-stranded oligonucleotides 
were synthesized using the described protocol above. An aliquot of $8 \mu 1$ of the master mix containing $1.0 \mu$ Poly d(I-C) $(1 \mu \mathrm{g} / \mu \mathrm{L}), 2.0 \mu \mathrm{l}$ of $5 \mathrm{X}$ EMSA Binding buffer, and $5 \mu \mathrm{l}$ nuclease-free water was added to each of two test tubes (control and high dehydration) and a $9 \mu \mathrm{l}$ aliquot of the master mix was added to two control tubes ("no probe" and "no protein"). The EMSA binding buffer consisted of $5 \mathrm{mM}$ Tris-HCl, $25 \mathrm{mM} \mathrm{NaCl}$, and 5 mM EDTA, and $25 \% \mathrm{v}: \mathrm{v}$ glycerol $(\mathrm{pH} 7.8)$. A total of $4 \mu \mathrm{g}$ of nuclear extract $($ at $1 \mu \mathrm{g} / \mu \mathrm{l})$ was added to the two test tubes and the "no probe" control and allowed to incubate for 5 minutes at room temperature. Following the incubation, $1 \mu$ of the double-stranded probe (10 ng) was added to all the tubes except for the "no probe" control. The tubes were incubated at $15^{\circ} \mathrm{C}$ for 30 minutes in a thermal cycler, forming a transcription factor DNA complex. In subsequent tests, a protein gradient was created using $16 \mu \mathrm{g}, 32 \mu \mathrm{g}$ or $40 \mu \mathrm{g}$ of nuclear extract (at $1 \mu \mathrm{g} / \mu \mathrm{l})$.

A $6.0 \%$ non-denaturing polyacrylamide gel was prepared to separate out the complex. The gel was made up of $0.5 \mathrm{~mL}$ of chilled 10X TBE $(0.89 \mathrm{M}$ Tris- $\mathrm{HCl}, 0.89 \mathrm{M}$ boric acid, 0.04 M EDTA, pH 8.0), 2 mL 30\% bis-acrylamide, $312 \mu 180 \%$ glycerol, 7

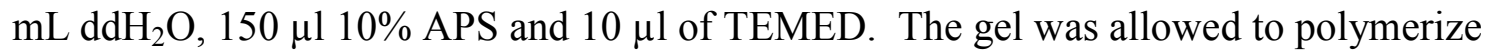
and then pre-run with $0.5 \mathrm{X} \mathrm{TBE}$ at $120 \mathrm{~V}$ for 10 minutes in the electrophoresis apparatus at $4^{\circ} \mathrm{C}$.

The prepared samples were mixed with $1 \mu 1$ of 6X DNA loading dye (BioShop) and $10 \mu 1$ of each sample was loaded into the gel. The gel was run at $120 \mathrm{~V}$ for 40 minutes at $4^{\circ} \mathrm{C}$. The complex was then transferred onto a Biodyne B nylon membrane (Pall Corporation) in $0.5 \mathrm{X}$ TBE buffer at a constant $300 \mathrm{~mA}$ for 30 minutes. Following the transfer, the membrane was placed between two dry sheets of filter paper and placed in a 
dry oven (at $80^{\circ} \mathrm{C}$ ) to allow the oligonucleotides to fix on the membrane. The membranes were transferred to a clean membrane box and incubated for $15 \mathrm{~min}$ in $5 \mathrm{~mL}$ of $1 \mathrm{X}$ blocking buffer (Panomics Catalogue \#: CS7535) at room temperature. Following this step, $1 \mathrm{~mL}$ of the $1 \mathrm{X}$ blocking buffer was removed and combined with $5 \mu \mathrm{l}$ of streptavidin-HRP and then added back to the membrane box. The membrane was allowed to rock for an additional $15 \mathrm{~min}$ at room temperature. The blocking solution was removed and the membrane was washed for 30 min with $1 \mathrm{X}$ washing buffer (Panomics Catalogue \#: CS 7537). The membrane was developed using enhanced chemiluminescence $\left(\mathrm{H}_{2} \mathrm{O}_{2}\right.$ and ECL) reagents using a Chemi-Genius BioImaging system (Syngene, Frederick, MD)

\section{Stem loop PCR}

A $5.0 \mu \mathrm{L}$ aliquot of total RNA $(1 \mu \mathrm{g} / \mu \mathrm{L})$ was incubated with $1.0 \mu \mathrm{L}$ of $250 \mathrm{nM}$ microRNA-specific stem-loop primer. The reaction was incubated in a thermal cycler at $95^{\circ} \mathrm{C}$ for $5 \mathrm{~min}$ to denature the RNA and $60^{\circ} \mathrm{C}$ for an additional 5 minutes to anneal the stem loop primer. The tubes were then placed in ice for 1 minute and $4 \mu \mathrm{L}$ of $5 \mathrm{X}$ first strand buffer, $2 \mu \mathrm{L}$ of $0.1 \mathrm{M} \mathrm{DTT}, 1 \mu \mathrm{L}$ of dNTP mixture ( $25 \mathrm{mM}$ each) and $1 \mu \mathrm{L}$ of MMLV reverse transcriptase were added to each reaction. The incubation procedure was as follows: $30 \mathrm{~min}$ at $16^{\circ} \mathrm{C}, 30 \mathrm{~min}$ at $42^{\circ} \mathrm{C}$ and $5 \mathrm{~min}$ at $85^{\circ} \mathrm{C}$. The double-stranded microRNA product was serially diluted to $10^{-1}$ and $10^{-2}$ and stored at $-20{ }^{\circ} \mathrm{C}$.

To amplify the microRNA product tubes were prepared containing $12.75 \mu \mathrm{L}$ of sterile water, $5 \mu \mathrm{L}$ of RT product, $2 \mu \mathrm{L}$ of primer mixture $(25 \mu \mathrm{M}$ forward, $25 \mu \mathrm{M}$ universal reverse), $2.5 \mu \mathrm{L}$ of 10X PCR buffer (Invitrogen), $1.25 \mu \mathrm{L}$ of $\mathrm{MgCl}_{2}(50 \mathrm{mM})$, $0.5 \mu \mathrm{L}$ of dNTPs (25 mM each) and $1 \mu \mathrm{L}$ of Taq polymerase. The universal primer 
sequence was 5'-CTCACAGTACGTTGGTATCCTTGTG-3'. A summary of the stem and forward microRNA primers used is given in Table 2.4. The reactions were then incubated for 10 minutes at $95^{\circ} \mathrm{C}$ followed by 33 cycles consisting of 10 seconds at $95^{\circ} \mathrm{C}$ and 15 seconds at $60^{\circ} \mathrm{C}$. Each PCR product was electrophoretically separated on a $2 \%$ agarose gel (Bioshop) in the presence of a Syber-Green I loading dye. Samples were run at $130 \mathrm{~V}$ for 20 minutes with $1 \mathrm{X}$ TAE buffer diluted from $50 \mathrm{X}$ TAE buffer ( 2 M Tris$\mathrm{HCl}, 1 \mathrm{M}$ acetic acid, 0.05 $\mathrm{M}$ EDTA, adjusted to $\mathrm{pH} 8.5)$. The bands were visualized under UV light using the ChemiGenius Bio Imaging System and the band densities were quantified using the GeneTools software. PCR products were sequenced by BioBasic (Markham, ON, Canada).The sequences were verified as encoding the microRNA using the program BLASTN (http://www.ncbi.nlm.nih.gov/blast) at the NIH.

\section{Prediction of microRNA binding}

The ability of the selected microRNAs to bind their proposed target genes was determined using the conservation of the microRNA seed-pairing sites and the thermodynamics of the RNA duplex formation. The conservation of all microRNAs was determined using TargetScan 5.2, a program which matches a particular microRNA to potential targets using only the 7 nucleotides of the seed region and compares it to several model organism. The binding of $X$. laevis miRNAs was predicted based on the conserved sequences found in the 3'UTR of six mammal and one bird species: human (Homo sapiens), common chimpanzee (Pan troglodytes), nine-banded armadillo (Dasypus novemcinctus), lesser hedgehog tenrec (Echinops telfair), house mouse (Mus musculus), red junglefowl (Gallus gallus), and a brown rat (Rattus 
norvegicus). To determine the thermodynamic stability, the RNAhybrid algorithm was used to statistically predict the most energetically favourable hybridization sites. A cutoff biding energy of -18 kJ was used to confirm the binding likelihood of the microRNA of interest to its target gene.

Table 2.4: Stem loop and forward microRNA primers that were used in the study.

\begin{tabular}{|l|l|l|}
\hline MicroRNA & Stem Loop Primer(5' $\left.\rightarrow 3^{\prime}\right)$ & Forward Primer(5' $\left.\rightarrow 3^{\prime}\right)$ \\
\hline $\begin{array}{l}\text { Xla-mir- } \\
15 \mathrm{a}\end{array}$ & $\begin{array}{l}\text { CTCACAGTACGTTGGTATCCTTGTGA } \\
\text { TGTTCGATGCCATATTGTACTGTGAG } \\
\text { CACAAACC }\end{array}$ & $\begin{array}{l}\text { ACACTCCAGCTGGGTA } \\
\text { GCAGCACATAATGG }\end{array}$ \\
\hline Xla-mir-16 & $\begin{array}{l}\text { CTCACAGTACGTTGGTATCCTTGTGA } \\
\text { TGTTCGATGCCATATTGTACTGTGAG } \\
\text { CGCCAATA }\end{array}$ & $\begin{array}{l}\text { ACACTCCAGCTGGGTA } \\
\text { GCAGCACGTAAATA }\end{array}$ \\
\hline $\begin{array}{l}\text { Xla-mir- } \\
19 \mathrm{~b}\end{array}$ & $\begin{array}{l}\text { CTCACAGTACGTTGGTATCCTTGTGA } \\
\text { TGTTCGATGCCATATTGTACTGTGAG } \\
\text { TCAGTTTT }\end{array}$ & $\begin{array}{l}\text { ACACTCCAGCTGGGTG } \\
\text { TGCAAATCCATGCAA }\end{array}$ \\
\hline Xla-mir-21 & $\begin{array}{l}\text { CTCACAGTACGTTGGTATCCTTGTGA } \\
\text { TGTTCGATGCCATATTGTACTGTGAG } \\
\text { TCAACATC }\end{array}$ & $\begin{array}{l}\text { ACACTCCAGCTGGGTA } \\
\text { GCTTATCAGACTGA }\end{array}$ \\
\hline $\begin{array}{l}\text { Xla-mir- } \\
\text { 92a }\end{array}$ & $\begin{array}{l}\text { CTCACAGTACGTTGGTATCCTTGTGA } \\
\text { TGTTCGATGCCATATTGTACTGTGAG } \\
\text { CAGGCCGG }\end{array}$ & $\begin{array}{l}\text { ACACTCCAGCTGGGTA } \\
\text { TTGCACTTGTCCC }\end{array}$ \\
\hline $\begin{array}{l}\text { Xla-mir- } \\
101\end{array}$ & $\begin{array}{l}\text { CTCACAGTACGTTGGTATCCTTGTGA } \\
\text { TGTTCGATGCCATATTGTACTGTGAG } \\
\text { TCAGTTAT }\end{array}$ & $\begin{array}{l}\text { ACACTCCAGCTGGGTA } \\
\text { CAGTACTGTGAT }\end{array}$ \\
\hline $\begin{array}{l}\text { Xla-mir- } \\
155\end{array}$ & $\begin{array}{l}\text { CTCACAGTACGTTGGTATCCTTGTGA } \\
\text { TGTTCGATGCCATATTGTACTGTGAG } \\
\text { CCCCTATC }\end{array}$ & $\begin{array}{l}\text { ACACTCCAGCTGGGTT } \\
\text { AATGCTAATTGTGA }\end{array}$ \\
\hline
\end{tabular}

\section{Statistical analysis}

Band densities for all the genes on agarose gels and band densities for all proteins on chemiluminescent immunoblots were visualized using the Chemi-Genius BioImaging system (Syngene, Frederick, MD) and quantified using Gene Tools software. All PCR bands representing genes of interest were normalized against the corresponding density 
of the $\alpha$-tubulin band amplified from the same cDNA sample. Immunoblots were normalized against the summed intensities of a group of Coomassie-stained proteins from the same lane; these were chosen because they did not show variation between different experimental states and were not located close to the protein bands of interest.

Absorbance values obtained in the ELISA experiments were standardized by subtracting the mean of the negative controls (wells containing no nuclear extract) from the experimental values.

Data are expressed as means \pm standard error of the mean $(\mathrm{SEM}), \mathrm{n}=3-4$ independent samples. Statistical testing of standardized band intensities used one-way ANOVA and a post-hoc test (Student-Newman-Keuls). 


\section{Chapter 3:}

Expression of NF- $\kappa B$ transcription factor and its anti-apoptotic targets in liver and skeletal muscle of dehydrated $X$. laevis. 


\section{Introduction}

Nuclear factor- $\kappa \mathrm{B}(\mathrm{NF}-\kappa \mathrm{B})$ was first discovered in 1986 when it was described as a transcription factor that enhances the expression of a gene in Beta cells, coding for the $\kappa$ light chain of immunoglobin, a protein responsible for identifying foreign antigens. The Rel/NF- $\mathrm{B}$ family contains five members: p50/p105, p52/p100, RelA (p65), c-Rel and RelB all of which contain a structurally conserved 300 amino acid sequence called the REL homology domain (Baldwin, 1996). These structurally related proteins are able to form homodimers and heterodimers, the most common dimer in mammals being RelA-

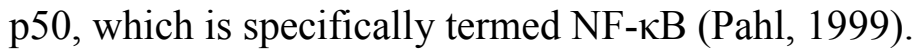

NF- $\kappa \mathrm{B}$ was first recognized for its involvement in the response by cells to a variety of antigens such as bacteria and viruses. The role of NF- $\kappa \mathrm{B}$ in the host immune response has been well studied, as its activity is induced by foreign antigens and its target genes include cytokines and receptors required for immune recognition (Baldwin, 1996). As a result, the NF- $\mathrm{KB}$ transcription factor has earned the title of the "central mediator of the human immune response". However, it is now known that the roles of this transcription factor extend beyond the defensive response to antigens. Increased NF- $\kappa \mathrm{B}$ activity has been observed in response to (1) physiological stress conditions; such as ischemia/reperfusion, liver regeneration, hemorrhagic shock, (2) physical stress; such as irradiation and oxidative stress, and (3) other stresses; such as environmental hazards and therapeutic drugs (Paul, 1999). It has recently been determined that NF- $\kappa \mathrm{B}$ is an important marker of cancer as examples of its target genes include those with a role in cell survival (Bcl-xL), proliferation (cyclin D1), angiogenesis (VEGF), and metastasis (MMP), among others (Nishikori, 2005). Increased NF- $\kappa$ B activity and DNA binding has 
been described in many human cancers such as breast cancer (Biswas et al, 2004), melanoma (Ueda and Richmond, 2006) and pancreatic cancer (Fujioka, et al, 2003), in addition to several haematological malignancies (Basse'res and Baldwin, 2006). Given the strong relationship between NF- $\mathrm{kB}$ expression and tumor growth, studies in the area are currently directed at inhibiting NF- $\mathrm{KB}$ activity using various compounds to prevent cancer cell development. Although more studies are required, the possibility that blockers of NF- $\mathrm{\kappa B}$ will be used in cancer therapy in the future appears to be promising (Basse'res and Baldwin, 2006).

A family of proteins collectively called inhibitors of NF- $\kappa B$ (IкB) regulate NF- $\kappa B$

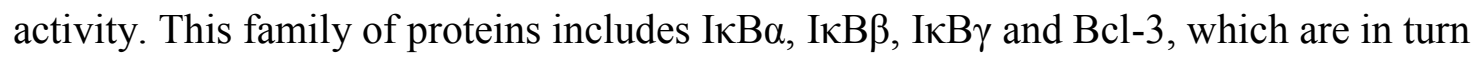
regulated by the IкB kinase (IKK) complex composed of three subunits; " $\alpha$ " and " $\beta$ " catalytic subunits and a " $\gamma$ " regulatory subunit. To date, two major pathways of NF- $\kappa B$ activation have been described called the classical and the alternative pathways, which are triggered by separate signals and serve different functions in the cell (Figure 3.1; Senftleben et al, 2001).

In the classical signalling NF- $\mathrm{kB}$ pathway, a stress stimulus initiates a reaction cascade that activates the IKK complex, which acts through the IKK $\beta$ subunit to phosphorylate IкB $\alpha$ at Ser32 and Ser36, signalling its degradation by the action of $26 \mathrm{~S}$ protease. The NF- $\kappa B$ dimer, most commonly p50-RelA in this pathway, is released from its inhibitory control by $\mathrm{I} \kappa \mathrm{B} \alpha$, and can translocate to the nucleus (Senftleben et al, 2001).The activation of the IKK complex in this pathway is initiated by proinflammatory cytokines and pathogen-associated molecular patterns (PAMPs), working through different receptors belonging to the tumor necrosis factor receptor (TNFR) and Toll-like 
receptor (TLR)-interleukin-1 (IL-1) receptor (IL-1R) superfamilies (Bonizzi and Karin, 2004).

Where the classical pathway is mainly responsible for the protection against infection and inflammation, the alternative pathway controls for the formation of B cells and the development of secondary lymphoid organs such as the lymph nodes (Senftleben et al, 2001). The IKK $\alpha$ homodimer is activated through the lymphotoxin$\beta$ receptor (LTBR), B-cell activating factor belonging to the TNF family (BAFF) receptor (BAFF-R) or CD40 (Bonizzi and Karin, 2004). The active IKK $\alpha$ phosphorylates p100/NFKB2 catalyzing the proteosomal degradation of the inhibitory C-terminal domain of $\mathrm{p} 100$, which leads to the release of the active p52 subunit. Once released from their inhibitory controls, the heterodimer enter the nucleus and binds to the $\mathrm{kB}$ sites of its target genes, increasing their rate of transcription (Bonizzi and Karin, 2004).

The present chapter evaluated the role of $\mathrm{NF}-\kappa \mathrm{B}$ in dehydration stress response in X. laevis in both medium and highly dehydrated liver and skeletal muscle tissues. This study focuses on the role of this transcription factor in protecting the dehydrated organs from apoptosis through the analysis of its pro-survival downstream targets; cellular inhibitor of apoptosis 1 (c-IAP1) and B-cell lymphoma-extra-large $(B c l-x l)$.

The inhibitors of apoptosis family consists of six members which are characterized by a short domain, about 70 amino acids in length, called the baculoviral IAP repeat (BIR). All members of the family, which includes NAIP, c-IAP1/HIAP-2, cIAP2/HIAP-1, XIAP/hILP, Survivin, and BRUCE, contain either 2 or 3 copies of this novel domain (Deveraux and Reed, 1999). IAP protein levels are elevated in response to 
cell death stimuli such as tumor necrosis factor (TNF) at which time they exert their protective function by directly inhibiting caspases (Duckett et al, 1996).

A.

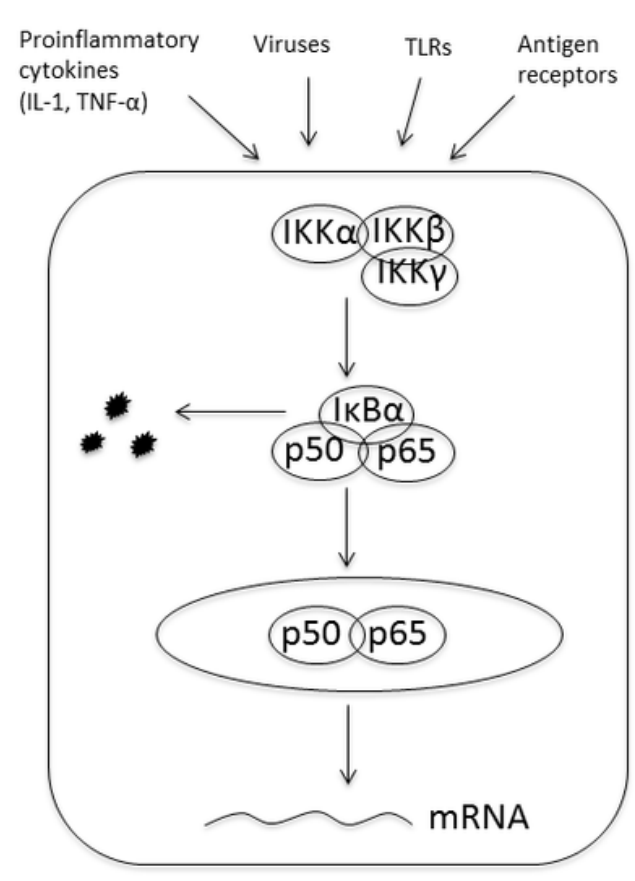

B.

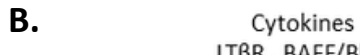

Cytokines
LTRR, BAFF/Blys

and CD4OL

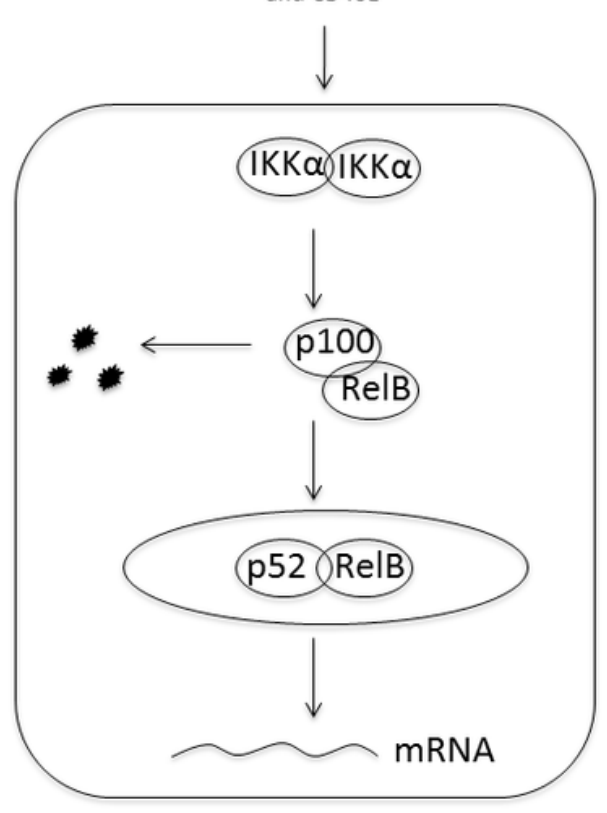

Figure 3.1: The classical and alternative signalling pathways of NF- $\kappa \mathrm{B}$ activation. (A) In the classical pathway a stress signal such as a virus, initiates the activation of the IKK complex through interaction with certain receptors such as TNFR or TLR. The activated IKK $\beta$ subunit phosphorylates I $\mathrm{B} \alpha$ which leads to its degradation and the release of the p50/p65 dimer from its inhibitory control. (B) In the alternative pathway, cytokines acting through the $L T \beta R, B A F F-R$ or CD40 receptors, activate the IKKa homodimer which phosphorylates the p100 precursor catalyzing the proteosomal degradation of the inhibitory C-terminal domain, releasing the active p52 subunit. Both dimers proceed to enter the nucleus and bind to the promoter regions of their target genes, enhancing their transcription (figure adapted from Bonizzi and Karin, 2004). 
Research on the detailed mechanism(s) via which IAPs are able to protect the cell against apoptotic signals is still ongoing and once described, it may greatly advance the field of cancer research. The current accepted mechanism of IAP action is by directly binding caspase proteins and inhibiting their function. Activated caspase proteins initiate the cell death response by cleaving vital proteins such as those with functions in cytoskeleton and nucleus composition (ex. actin and lamin A), cell-cycle arrest (ex. p21), and components of the DNA repair machinery (ex. PARP) (Earnshaw et al, 1999). An important member of the IAP family is c-IAP1, which is able to directly bind caspase -3, -7 and -9 at its BIR domain, inhibiting their destructive action ( $\mathrm{Li}$ and $\mathrm{Li}, 2000)$. Relative levels of c-IAP1 protein were evaluated in this study to provide evidence of its activation during the stress of dehydration.

The second NF- $\mathrm{kB}$ target investigated in this chapter is Bcl-xL, a member of the Bcl-2 family of proteins, which includes 14 other family members all of which contain at least one of four conserved motifs known as Bcl-2 homology domains (BH1- BH4). This related group of proteins contains both anti-apoptotic (Bcl-2, Bcl-xL, Bcl-w, etc) and proapoptotic members (Bax, BAD, Bak etc), which can heterodimerize to oppose each other's function (Adams and Cory, 1998). Bcl-xL is localized to the cytoplasmic face of the mitochondrial membrane, the nuclear envelope, the endoplasmic reticulum, as well as, in its soluble form, to the cytoplasm (Schendel et al, 1998). It has been found that Bcl-xL is able to bind CED-4 type molecules such as apoptotic protease activating factor 1 (Apaf-1), preventing the activation of caspase-9. Under conditions of stress, Apaf-1 binds cytochrome $\mathrm{c}$ and dATP forming an apoptosome that proceeds to bind and cleave 
caspase-9 to its active form allowing it to initiate a reaction cascade that results in cell death (Borner, 2003).

A number of NF- $\mathrm{BB}$ downstream targets include those with pro-apoptotic properties, including the tumor suppressing transcription factor p53 (Wu and Lozano, 1994). This chapter investigated whether NF- $\mathrm{B}$ discriminately activated its downstream targets, enhancing the transcription of only those with functions in cell survival. Many studies in the area of human cancer have focused on this transcription factor as the majority of human cancers were found to have a mutated p53 protein. The normal function of p53 is to suppress abnormal cell growth by up-regulating genes whose protein products are involved in initiating the cell death program or cell cycle arrest (Gottlieb and Oren, 1998). Examples of its downstream targets are p21; responsible for the halt of cell division, Bax; a protein which promotes cell suicide, and death receptors such as CD95/Fas/Apo1 (Levine, 1997). Wu and Lozano (1994) have found that TNF- $\alpha$ binding

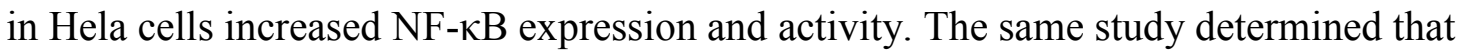
$\mathrm{NF}-\kappa \mathrm{B}$ bound to the p53 promoter site, amplifying its expression, supposedly to arrest the cell cycle to allow the cell to repair the damaged induced by stress (Wu and Luzano. 1994). Here, p53 expression was evaluated to better characterize the unique cellular response to the stress of dehydration.

In this chapter, evidence for the regulated activation of NF- $\kappa \mathrm{B}$ during $X$. laevis dehydration is evaluated in two tissues, liver and skeletal muscle, through the examination of relative abundance of $\mathrm{NF}-\kappa \mathrm{B}$ subunits in total and nuclear extracts along with a study of their DNA binding ability. In addition, two anti-apoptotic targets, Bcl-xL and c-IAP, were studied by measuring their relative transcript and protein levels under 
stress conditions compared to controls. Finally, levels of the apoptotic transcription factor, $\mathrm{p} 53$, were evaluated to determine whether NF- $\mathrm{kB}$ discriminately activates antiapoptotic genes during dehydration.

\section{Results}

\section{Analysis of $N F-\kappa B$ subunits protein levels in liver}

Relative amounts of NF- $\mathrm{BB}$ p50 and p65 subunits as well as their phosphorylated forms were compared in control, medium dehydration (MD) and high dehydration (HD) conditions in the liver tissue of $X$. laevis frogs via immunoblotting (Figure 3.2). The p50 and p65 antibodies each crossreacted with single bands on immunoblots at $50 \mathrm{kDa}$ and 65 $\mathrm{kDa}$, respectively. Relative total protein levels the p65 subunit dropped significantly to 57 $\pm 0.07 \%$ of control values $(P<0.05)$ in the high dehydration condition while levels of the p50 subunit remained the same between the three conditions. Relative phosphorylation of p65 (Ser 529) increased significantly in HD by $1.5 \pm 0.1$ fold, whereas the amount of phosphorylation of p50 (Ser 337) increased significantly in MD and HD by $4.12 \pm 0.39$ and $4.54 \pm 0.48$ fold, respectively $(P<0.05)$.

\section{Analysis of IאB $\alpha$ and phosphorylated IkB $\alpha$ in liver}

Immunoblotting was also carried out to analyse the relative protein levels of IкB $\alpha$

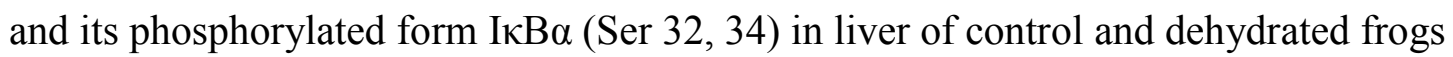
(Figure 3.2). Total protein levels of I $\mathrm{I} \mathrm{B} \alpha$ did not differ between control, MD and HD conditions. However, relative phosphorylation protein levels of $\operatorname{I\kappa } \mathrm{B} \alpha(\operatorname{Ser} 32,34)$ increased significantly in MD by $2.0 \pm 0.1$ fold and rose further with higher dehydration 
to $2.7 \pm 0.3$ fold over controls, a value that was also significantly higher than MD $(P<0.05)$

\section{Analysis of nuclear translocation of NF-אB subunits in liver}

Nuclear extracts of liver from control and highly dehydrated Xenopus frogs were analysed to determine whether the p50 and p65 subunits translocated into the nucleus from the cytoplasm in response to dehydration stress. Figure 3.3 shows the relative levels of the phosphorylated forms of p65 (Ser 529) and p50 (Ser 337) in nuclear extracts from control versus HD conditions. The relative protein levels of both p65 (Ser 529) and p50 (Ser 337) significantly increased, to $1.96 \pm 0.04$ and $2.89 \pm 0.05$ respectively, in HD compared to control $(P<0.05)$.

\section{Analysing DNA-binding of NF-אB subunits in liver}

The relative amount of DNA-binding by the two NF- $\kappa B$ subunits in nuclear extracts was measured using an ELISA procedure. Figure 3.4 shows the relative binding of phosphorylated p65 (Ser 529) and p50 (Ser 337) in nuclear extracts of liver.

Significant increases in binding were seen under HD conditions, levels rising by $1.50 \pm$ 0.01 and $2.51 \pm 0.31$ fold, respectively, as compared to control values $(P<0.05)$. This increase in relative absorbance values indicates a significant increase in the amount of both phosphorylated subunits to the in the nucleus of liver cells under dehydrated conditions. 


\section{EMSA validation}

An EMSA procedure was used to further validate the DNA binding of NF- $\kappa$ B. To determine whether $X$. laevis NF- $\mathrm{BB}$ bound specifically to the designed probe representing the consensus DNA binding element, samples of liver nuclear extracts were incubated with double-stranded DNA probe and DNA-protein complexes were allowed to form and then separated on a polyacrylamide gel. Figure 3.5 shows an EMSA image of a gel run from incubations with $16 \mu \mathrm{g}, 32 \mu \mathrm{g}$ or $40 \mu \mathrm{g}$ of liver nuclear extract from control versus HD conditions, along with a "no protein" and "no probe" controls. The image shows a band corresponding to the NF- $\mathrm{B} / \mathrm{DNA}$ complex that does not appear in the two control lanes along with two bands at the bottom of each lane corresponding to unbound single stranded probe and unbound double stranded probe. Bands representing the DNA-protein complex increased in intensity with increasing concentration of nuclear extract. The presence of these three bands, corresponding to the DNA-protein complex and the unbound single and double stranded NF- $\mathrm{KB}$ probe, suggest that a shift from the low molecular weight unbound probe to the higher molecular weight protein/DNA complex was successfully produced.

\section{Analysis of transcript levels of $\mathrm{NF}-\kappa \mathrm{B}$ downstream targets in liver}

c-IAP1, Bcl-xL and p53 are all important downstream targets of NF- $\kappa$ B. Figure

3.6 shows the relative transcript levels of these genes assessed using RT-PCR in X. laevis liver from control, MD and HD conditions. Under both dehydration conditions $b c l-x l$ transcript levels increased significantly; values were $1.4 \pm 0.1$ fold higher than controls in the MD condition and $1.56 \pm 0.09$ fold higher in the HD condition compared to controls $(P<0.05)$. Both $c$-iapl and $p 53$ transcript expression levels increased only under HD, 
rising by $1.29 \pm 0.06$ and $2.90 \pm 0.24$ fold, respectively, as compared with controls $(P<0.05)$.

\section{Analysis of protein levels of NF-kB downstream targets in liver}

Xenopus liver extracts were analysed by immunoblotting for relative changes in the protein levels of c-IAP1, Bcl-xL, and p53 proteins in response to whole body dehydration (Figure 3.7). The polyclonal antibodies for c-IAP1, Bcl-xL and p53 each crossreacted with a single band at approximately $70 \mathrm{kDa}, 26 \mathrm{kDa}$, and $53 \mathrm{kDa}$, respectively. c-IAP1 and Bcl-xL protein levels significantly increased under both MD and HD conditions. c-IAP1 protein levels increased by $1.38 \pm 0.06$ and $1.64 \pm 0.21$ fold in MD and HD, respectively, whereas Bcl-xL protein increased by to $2.00 \pm 0.18$ and $1.84 \pm 0.23$ fold in MD and HD, respectively $(P<0.05)$. Surprisingly, relative protein levels of p53 decreased significantly in both MD and HD to $72 \pm 0.04 \%$ and $68 \pm 0.04 \%$ of control values, respectively $(P<0.05)$.

\section{Analysis of NF- $\mathrm{KB}$ subunit protein levels in skeletal muscle}

Relative expression levels of the two NF- $\mathrm{kB}$ subunits, p50 and p65, were also analysed in the skeletal muscle of $X$. laevis (Figure 3.8). No significant differences were found in the relative protein levels of total p50 or p65, as well as the content of phosphorylated p65 (Ser 529) under either MD or HD conditions, as compared to controls. However, relative levels of phosphorylated p50 (Ser 337) decreased significantly during $\mathrm{HD}$ to $66 \pm 2 \%$ of control values. 


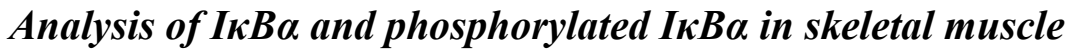

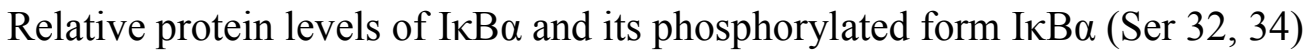
were also quantified in skeletal muscle (Figure 3.8). Total IкB $\alpha$ protein content did not differ between control, MD and HD conditions. However, significant changes were observed for phospho-IкB $\alpha$ (Ser 32,34), relative levels significantly decreasing under MD and HD conditions to $72 \pm 1 \%$ and $69 \pm 3 \%$ of control values, respectively $(P<0.05)$.

\section{Analysis of nuclear translocation of NF-кB subunits in skeletal muscle}

Nuclear extracts from skeletal muscle of control and highly dehydrated Xenopus frogs were analysed to determine whether there was evidence of nuclear translocation by the phosphorylated p65 and p50 subunits under stress conditions. Figure 3.9 shows the relative amounts of phosphorylated p65 (Ser 529) and p50 (Ser 337) in nuclear extracts as assessed by immunoblotting. There were no significant changes in the nuclear content of either subunit between control and HD conditions.

\section{Analysis of transcript levels of $\mathrm{NF}-\mathrm{\kappa B}$ downstream targets in skeletal muscle}

Relative abundance of $c$-iapl, bcl-xl and $p 53$ mRNA transcripts in skeletal muscle were assessed via RT-PCR. Figure 3.10 shows that both $c$-iap1 and $b c l$-xl expression levels increased significantly under both MD and HD conditions. Transcript levels of $c$ iap1 rose by $1.24 \pm 0.08$ fold in the MD condition and by $1.24 \pm 0.09$ fold in $\mathrm{HD}$ $(P<0.05)$. Similar results were observed in $b c l-x l$, expression levels increasing by $1.61 \pm$ 0.22 and $1.74 \pm 0.23$ fold in $\mathrm{MD}$ and $\mathrm{HD}$, respectively $(P<0.05)$. However, there were no 
significant changes in $p 53$ transcript levels in skeletal muscle tissue in response to dehydration.

\section{Analysis of protein levels of $\mathrm{NF}-\mathrm{\kappa B}$ downstream targets in skeletal muscle}

Immunoblotting was used to quantify the relative expression levels of NF- $\kappa \mathrm{B}$

downstream targets, c-IAP1, Bcl-xL and p53 (Figure 3.11). In all cases, antibodies

crossreacted with single bands for each protein at the expected molecular weights of 70

$\mathrm{kDa}$ for c-IAP1, $26 \mathrm{kDa}$ for $\mathrm{Bcl}-\mathrm{xL}$ and $53 \mathrm{kDa}$ for $\mathrm{p} 53$. Relative protein levels of $\mathrm{c}-\mathrm{IAP} 1$ rose by $1.94 \pm 0.03$ fold under HD conditions and Bcl-xL protein rose by $1.85 \pm 0.24$ fold, compared to control values $(P<0.05)$. However, p53 protein levels were unchanged under dehydrating conditions.

\section{Discussion}

The present chapter evaluated the effects of whole body dehydration on the expression of NF- $\mathrm{kB}$ and its anti-apoptotic downstream targets in X. laevis liver and skeletal muscle. The NF- $\mathrm{BB}$ transcription factor plays an important role in cell survival through its up-regulation of pro-survival (anti-apoptotic) downstream genes such as Bcl$\mathrm{xL}$ and c-IAP1 and suppression of pro-apoptotic genes such as p53 (Pahl, 1999).

Under normal conditions the $\mathrm{p} 50$ and $\mathrm{p} 65$ subunits of NF- $\mathrm{\kappa B}$ are found in the

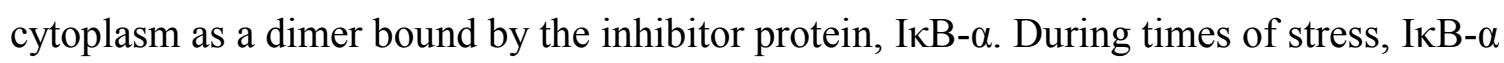
is phosphorylated at Ser 32 and 36 by the kinase, $\operatorname{IKK} \beta$, leading to the ubiquitination of I $\mathrm{B}-\alpha$ and its subsequent degradation by the proteosome. This frees NF- $\kappa \mathrm{B}$ to translocate into the nucleus and bind to genes possessing the appropriate NF- $\mathrm{kB}$ binding sequence in 
their promoter region (Wooten, 1999). NF- $\mathrm{kB}$ can be phosphorylated at several functional domains, optimizing both transcriptional activity and DNA binding. It has been reported that the nuclear translocation of NF- $\mathrm{kB}$ is not sufficient for DNA binding and in fact certain phosphorylation events need to take place for NF- $\mathrm{kB}$ to successfully bind to the promoters of its target genes (Wang et al, 2000). For example, cells lacking the required protein kinases (e.g. GSK3 $\beta$ ) to phosphorylate NF- $\mathrm{kB}$ subunits displayed

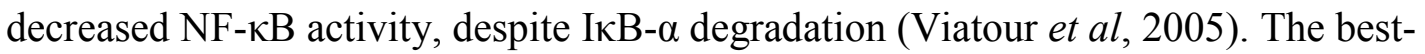
studied form of post-translational modifications to the NF- $\mathrm{KB}$ p50 subunit is the cyclic AMP-dependent protein kinase A (PKA) mediated phosphorylation of Ser 337. Studies have shown that the phosphorylation of p50 at this residue is essential for DNA binding of the NF- $\mathrm{kB}$ transcription factor since hypophosphorylated p50 subunits are able to enter the nucleus but fail to bind DNA (Hau et al, 2003). Multiple residues of p65 are also known to be phosphorylated and modify the activity of NF-kB (Viatour et al, 2005). The p65 phosphorylated form examined in this study is p65 (Ser 529) and was chosen because this phosphorylation event requires the degradation of the IкB- $\alpha$ inhibitor and thus is the most applicable to the activation pathway examined in this study. There is evidence to suggest that following IкB- $\alpha$ degradation, casein kinase II (CKII) phosphorylates p65 at serine 529, resulting in increased transcriptional potential (Wang et al, 2000).

In this study, liver from dehydrated Xenopus showed evidence of regulated NF$\kappa \mathrm{B}$ activation (Figure 3.2). Relative protein levels of phosphorylated p50 (Ser 337) increased by over 4-fold in liver from medium dehydrated and high dehydrated frogs compared to controls. Phosphorylated p65 (Ser 529) expression levels also increased significantly in HD by 1.5 fold compared to control animals. These increases in 
expression levels of NF- $\mathrm{NB}$ subunits coupled with an increase in the phosphorylation of I $\kappa \mathrm{B} \alpha$ at Ser 32/34 by 2-fold in MD compared to controls, and 2.7-fold in HD compared to $\mathrm{MD}$ and controls, indicates that a coordinated activation of the NF- $\mathrm{KB}$ transcription factor is taking place in liver under the stress of dehydration.

In addition to the increase in phosphorylation of $\mathrm{NF}-\kappa \mathrm{B}$ subunits in liver, evidence for the translocation of the transcription factor to the nucleus was provided by examining relative levels of NF- $\kappa \mathrm{B}$ in nuclear extracts (Figure 3.3). Since the relative phosphorylation of p50 (Ser 337) increased under both experimental conditions and levels of phospho-p65 (Ser 529) increased only in HD, the nuclear translocation experiments were focused on the high dehydration condition only.

Both phosphorylated forms of the NF-кB subunits, p50 (Ser 337) and p65 (Ser 529), were more abundant in the nucleus in the highly dehydrated condition compared to controls. Relative protein levels of p65 (Ser 529) increased by almost 2-fold, while levels of p50 (Ser 337) increased by 3-fold indicating that greater amounts of activated NF- $\mathrm{B}$ migrated to the nucleus in response to the stress of dehydration. Furthermore, DNA binding activity of NF- $\kappa \mathrm{B}$ was significantly enhanced in liver under high dehydration. This was evident from ELISA analyses where relative increases in DNA binding were seen for both p65 (Ser 529) and p50 (Ser 337) by as much as 1.5-fold and 2.5-fold, respectively, compared to controls (Figure 3.4). These data provide strong evidence for the increased transcriptional activity of NF- $\mathrm{BB}$ during the stress of dehydration.

The NF- $\kappa$ B response was different in skeletal muscle where p50 (total) and p65 (total and phosphorylated) did not differ significantly between conditions whereas phospho-p50 (Ser 337) expression levels decreased significantly in HD (Figure 3.8). 
Accordingly, the I $\kappa \mathrm{B} \alpha($ Ser 32,34$)$ expression levels were also significantly reduced in both medium and high dehydrated frogs compared to controls, providing an inhibitory mechanism to explain the lack of activation of the NF- $\mathrm{BB}$ transcription factor in skeletal muscle during dehydration. In addition, there was no evidence of increased translocation of phosphorylated NF- $\kappa$ B subunits to the nucleus under high dehydration conditions (Figure 3.9). These results highlight an important functional difference between two Xenopus tissues in terms of their NF- $\mathrm{KB}$ mediated response to stress.

Considering these results, it is clear that the phosphorylation of the p65 and p50 subunits is an important event required for NF- $\kappa \mathrm{B}$ activation and DNA binding. Although the total protein levels of both subunits remained the same in dehydrated liver tissues, the phosphorylated forms of these subunits were elevated in both total and nuclear extracts. This indicates that during times of stress, NF- $\kappa \mathrm{B}$ is activated in liver of $X$. laevis by both the regulated degradation of the I $\kappa \mathrm{B} \alpha$ inhibitor and the phosphorylation of important sites required for enhanced transcription factor activity.

Once activated, NF- $\kappa \mathrm{B}$ enters the nucleus where it promotes the transcription of target genes whose protein products are responsible for essential cellular functions. NF$\kappa \mathrm{B}$ targets include those with functions in antioxidant defence (MnSOD), immune response (several interleukin cytokines), cell survival (Bcl-xL) and other proteins that are vital for the cell's response to stress (Pahl, 1999). This chapter focuses on the role of NF$\kappa \mathrm{B}$ in promoting the onset of anti-apoptotic pathways in response to extracellular stimuli, which are transmitted from the cell membrane via cellular cascades. Evidence for the activation of anti-apoptotic pathways in liver and skeletal muscle of dehydrated frogs was collected through the study of c-IAP1 and Bcl-xL. 
The inhibitors of apoptosis family of proteins includes NAIP, c-IAP1/HIAP-2, cIAP2/HIAP-1, XIAP/hILP, Survivin, and BRUCE all of which possess either two or three copies of a motif termed the baculovirus IAP repeat (BIR; Deveraux and Reed, 1999). Overexpression of these proteins has been shown to suppress the cell death response when induced by a variety of stimuli such as tumor necrosis factor (Duckett et al, 1996). In this study, c-IAP1 was chosen to illustrate the cellular anti-apoptosis response since its expression levels are fairly similar in all tissues outside of the central nervous system and it is generally more abundant than c-IAP2 (Deveraux and Reed, 1999).

Although research on the exact mechanism by which IAPs inhibit apoptosis is still ongoing, it is well known that these proteins bind caspases directly, thereby inhibiting their apoptotic function. Caspases are a family of cysteine proteases that serve a major role in the cell's initiation of the cell death program including cleaving several intracellular polypeptides important to the structural integrity of the cell and components of the DNA repair machinery (Earnshaw et al, 1999). It has been shown that c-IAP1 can bind caspases $-3,-7$, and -9 (Roy et al, 1997). In this study c-iapl transcript levels increased by 1.3-fold in high dehydrated liver (Figure 3.6) and by 1.2-fold in both medium and high dehydrated skeletal muscle (Figure 3.10). Relative protein levels of cIAP1 increased to 1.4-fold and 1.6-fold under MD and HD conditions, respectively, in liver (Figure 3.7) and by almost 2-fold in HD in skeletal muscle (Figure 3.11).

The abundance of c-IAP1 protein does not directly match the changes in its mRNA levels in both tissues. In liver, transcript levels were elevated in HD but protein levels of c-IAP1 were significantly increased while the frogs were in the medium 
dehydration stage. This may be due to a regulation at the translational level (such as effects by microRNA or other controls on transcript availability to the ribosomal translation machinery) or at the post-translational where c-IAP1 protein degradation might be reduced under the stress condition (Ditzel and Meier, 2002).

One such post-translational mechanism of IAP regulation has recently been described. Recent studies have identified proteins that share an evolutionarily conserved IAP-binding motif (IBM) and are antagonists to the IAP proteins. Following a cell death signal, IBM proteins accumulate in the cytoplasm where they bind to IAPs, preventing them from directly inhibiting caspases. Following IAP binding, IBM proteins promote the ubiquitination and degradation of IAPs thereby breaking the cell's last line of defense. It is possible that one of the mechanisms by which cells resist apoptosis is by a decrease in basal IBM levels which allows for the accumulation of IAP proteins and subsequently cell survival (Ditzel and Meier, 2002).

In skeletal muscle, the increase in c-iap 1 transcript levels under both MD and HD conditions may have an added effect on the significant increase in its protein product in the high dehydration condition. Considering the significant elevation of c-IAP1 protein levels, it is apparent that it serves a major role in the stress response in Xenopus liver and skeletal muscle tissues. The lack of a complete correlation between the transcript and protein levels of c-IAP1 in these tissues is interesting, and requires further investigation.

Further evidence for the activation of anti-apoptotic pathways during dehydration stress was obtained by studying the relative expression levels of Bcl-xL. This protein belongs to the Bcl-2 family of proteins along with 14 other family members characterised by containing 1-4 conversed motifs termed the Bcl-2 homology domains (Adams and 
Cory, 1998). When an apoptosis program is initiated in a cell, a pro-apoptotic protein called apoptotic protease activating factor 1 (Apaf-1) binds cytochrome $\mathrm{c}$ and dATP forming an apoptosome, which is then able to splice caspase-9 to its active form. Bcl-xL opposes this sequence of reactions by binding Apaf-1, which prevents it from activating caspase-9 and propagating the cell death signaling cascade (Borner, 2003).

In the present study, relative transcript levels of $b c l-x l$ increased in liver of both experimental groups by 1.4 -fold and 1.6-fold in the medium dehydrated and the high dehydrated conditions, respectively (Figure 3.6). The relative concentration of Bcl-xL protein reflected the changes in relative mRNA levels since protein levels increased by 2fold in medium dehydrated frogs and by 1.8-fold in highly dehydrated frogs (Figure 3.7). Similar results were seen in skeletal muscle since $b c l-x l$ mRNA levels increased by 1.6fold in MD and 1.7-fold in HD while protein levels increased by 1.9-fold in HD only (Figure 3.10 and 3.11). This evidence suggests that $b c l-x l$ gene expression is an early response to the stress of dehydration since relative levels increased under MD in both liver and skeletal muscle. This enhanced level of $b c l-x l$ transcription correlates well with increased $\mathrm{Bcl}-\mathrm{xL}$ protein levels suggesting that dehydration stress requires the activation of pro-survival pathways.

Finally, the third NF- $\mathrm{kB}$ downstream target examined in this study was the tumor suppressing transcription factor p53 (Wu and Lozano, 1994). The wild type protein plays a major role in reducing tumour growth and the majority of human cancers are marked with an altered activity of p53 (Gottlieb and Oren, 1998). The downstream targets of p53 involve those responsible for cell cycle arrest, such as p21 and those triggering cell suicide, through the transcription of pro-apoptotic proteins, such as Bax and cell death 
receptors such as CD95/Fas/Apo1. Unexpectedly, transcript levels of p53 increased by almost 3-fold in high dehydrated liver compared to controls (Figure 3.6). However, the relative protein levels of p53 did not match its transcript levels as protein levels significantly decreased in both conditions to about $70 \%$ of control levels. In normal cells, p53 protein is kept at a low concentration because of its short half-life and expression levels increase in response to stressful conditions (Levine, 1997). It appears that relative levels of the p53 transcription factor were depressed even further under dehydration conditions compared to controls, perhaps to allow for a more efficient action of the prosurvival pathways. Transcription of the $p 53$ gene appears to be activated in HD as evidenced by the increased levels of $p 53$ mRNA. This enhanced level of transcription may be a result of NF- $\mathrm{NB}$ activation or triggered in response to cellular stimuli such as DNA damage or the presence of DNA repair intermediates (Levine, 1997). This enhanced transcriptional state is not, however, reflected in the relative protein levels and suggests a negative regulation at the translational level. This possibility will be further investigated in Chapter 5. In skeletal muscle, however, relative transcript and protein levels of p53 remained unchanged during dehydration.

Overall, there is evidence to indicate that under the stress of dehydration in $X$. laevis liver, NF- $\kappa \mathrm{B}$ activity is increased in a regulated manner through the degradation of its inhibitor IкB- $\alpha$ and the phosphorylation of important sites in its structure.

Furthermore, DNA binding experiments showed an increased presence of phosphorylated NF- $\kappa \mathrm{B}$ subunits in the nucleus under stress conditions compared to controls. Interestingly, the anti-apoptotic downstream targets; c-IAP1 and Bcl-xL, were found to increase under dehydrated conditions in both liver and skeletal muscle, which did not 
display evidence of regulated $\mathrm{NF}-\kappa \mathrm{B}$ activation. Thus, the data described in this chapter presents three conclusions; (1) NF- $\mathrm{BB}$ transcription factor activity is increased in $X$. laevis liver under dehydrated conditions, (2) anti-apoptotic proteins are actively transcribed and translated during the stress of dehydration in both liver and skeletal muscle of Xenopus frogs, and (3) these increases seem to be independent of NF- $\kappa \mathrm{B}$ activation and DNA binding at least in skeletal muscle. Since anti-apoptotic targets are activated in both liver and muscle tissues regardless of the activation of the NF- $\kappa \mathrm{B}$ transcription factor, further experiments were carried out to examine additional activation pathways. The STAT family of transcription factors also includes c-IAP and Bcl-xL as downstream targets. The expression and activation of this pathway is examined in Chapter 4. 
A.

B.
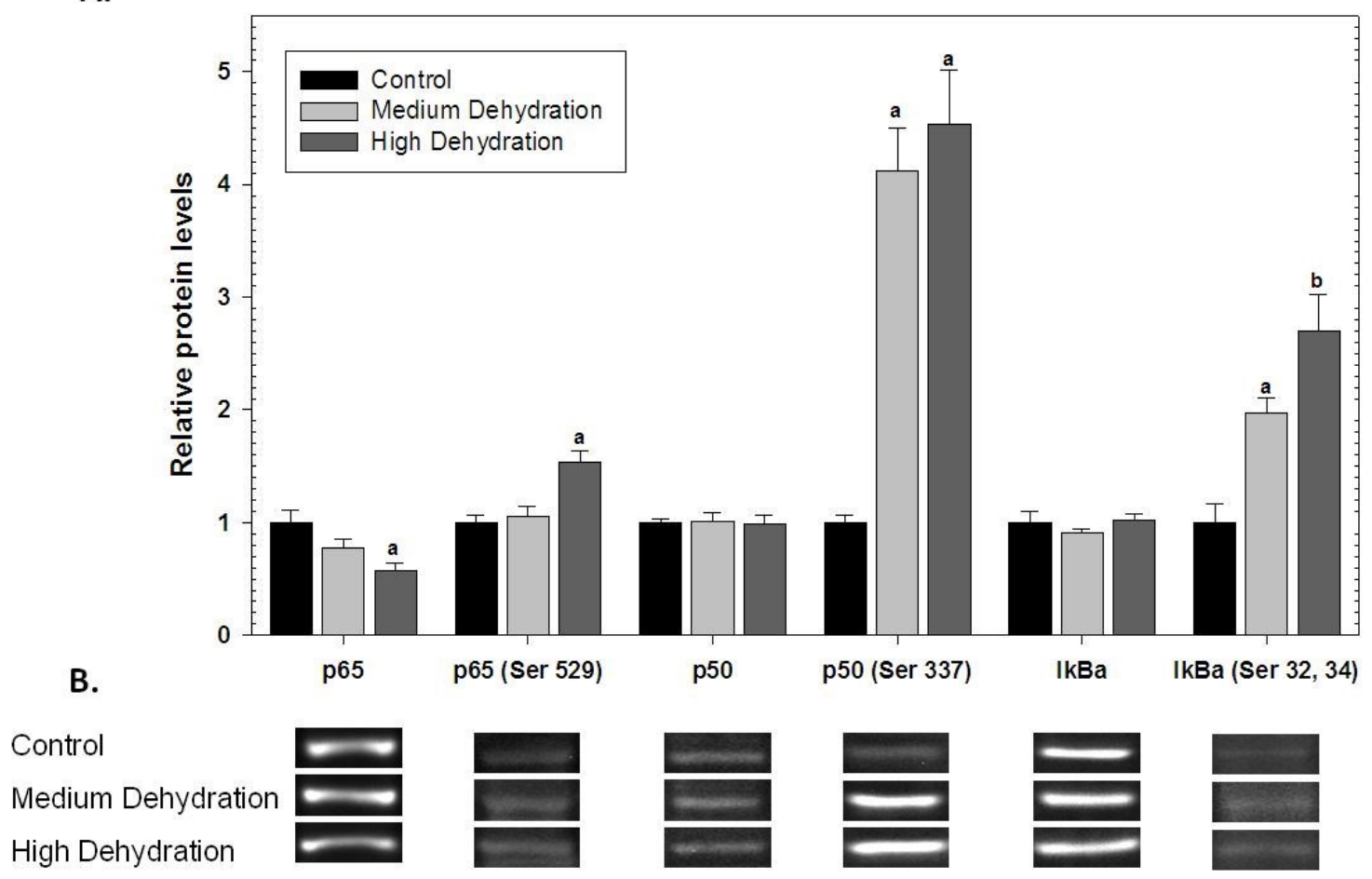

Figure 3.2: (A) Relative changes in protein expression of NF- $\kappa B$ transcription factor subunits, p65 and p50, and their phosphorylated forms, p65 (Ser 529) and p50 (Ser 337) in liver tissue during medium and high dehydration of $X$. laevis. Figure also shows the relative changes in the $\mathrm{NF}-\kappa \mathrm{B}$ inhibitor $\mathrm{I} \kappa \mathrm{B} \alpha$ and its phosphorylated form $\mathrm{I} \kappa \mathrm{B} \alpha$ (Ser 32 , 34). Data are means $\pm S E M, n=4$ independent trials on tissue samples from different animals. "a" - Significantly different from the control values; "b" - significantly different from medium dehydration, $(P<0.05)$. (B) Western blots showing representative bands. 
A.

B.

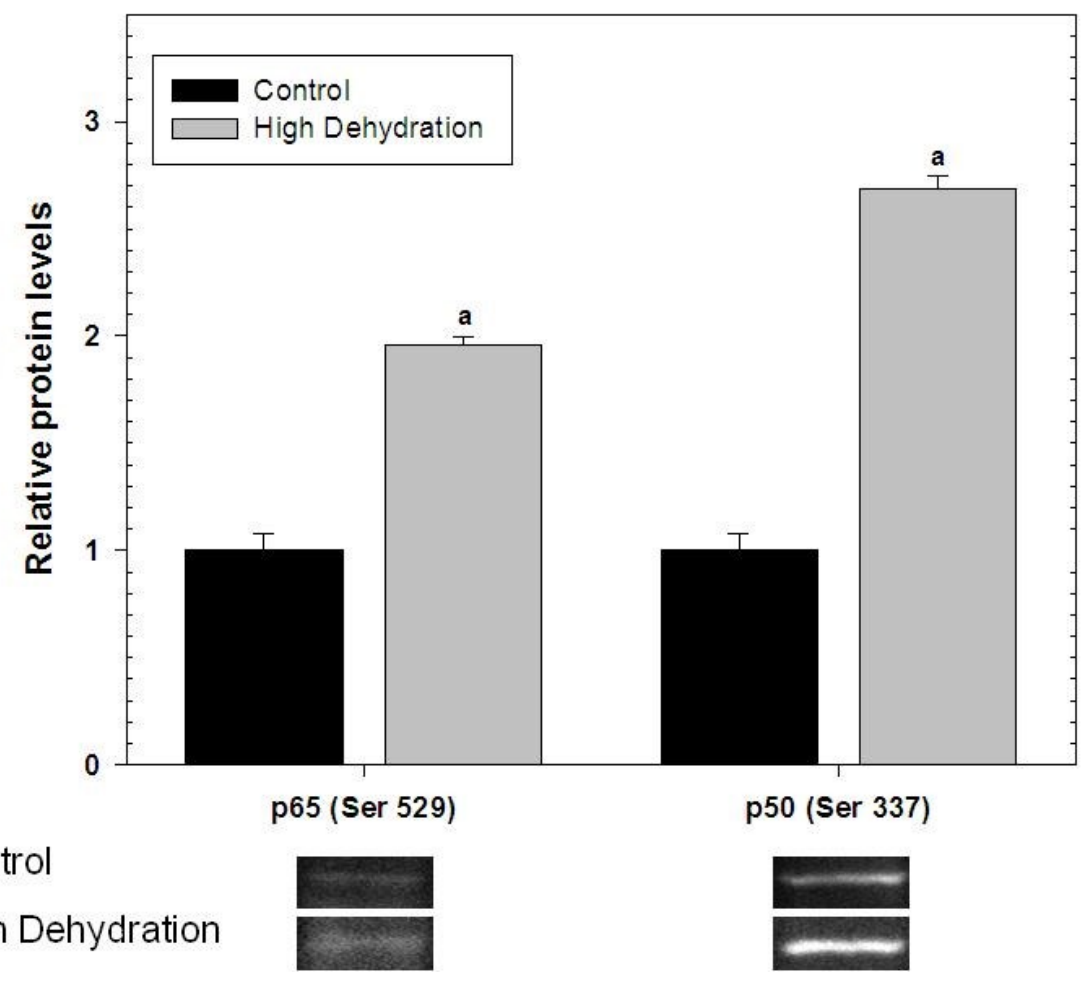

Figure 3.3: (A) Relative protein levels of p65 (Ser 529) and p50 (Ser 337) in nuclear extracts of liver from control and high dehydration $X$. laevis. Data are means $\pm \mathrm{SEM}$ for $\mathrm{n}=4$ independent trials on tissue samples from different animals. "a" - Significantly different from the control values $(P<0.05)$. (B) Western blots showing representative bands. 


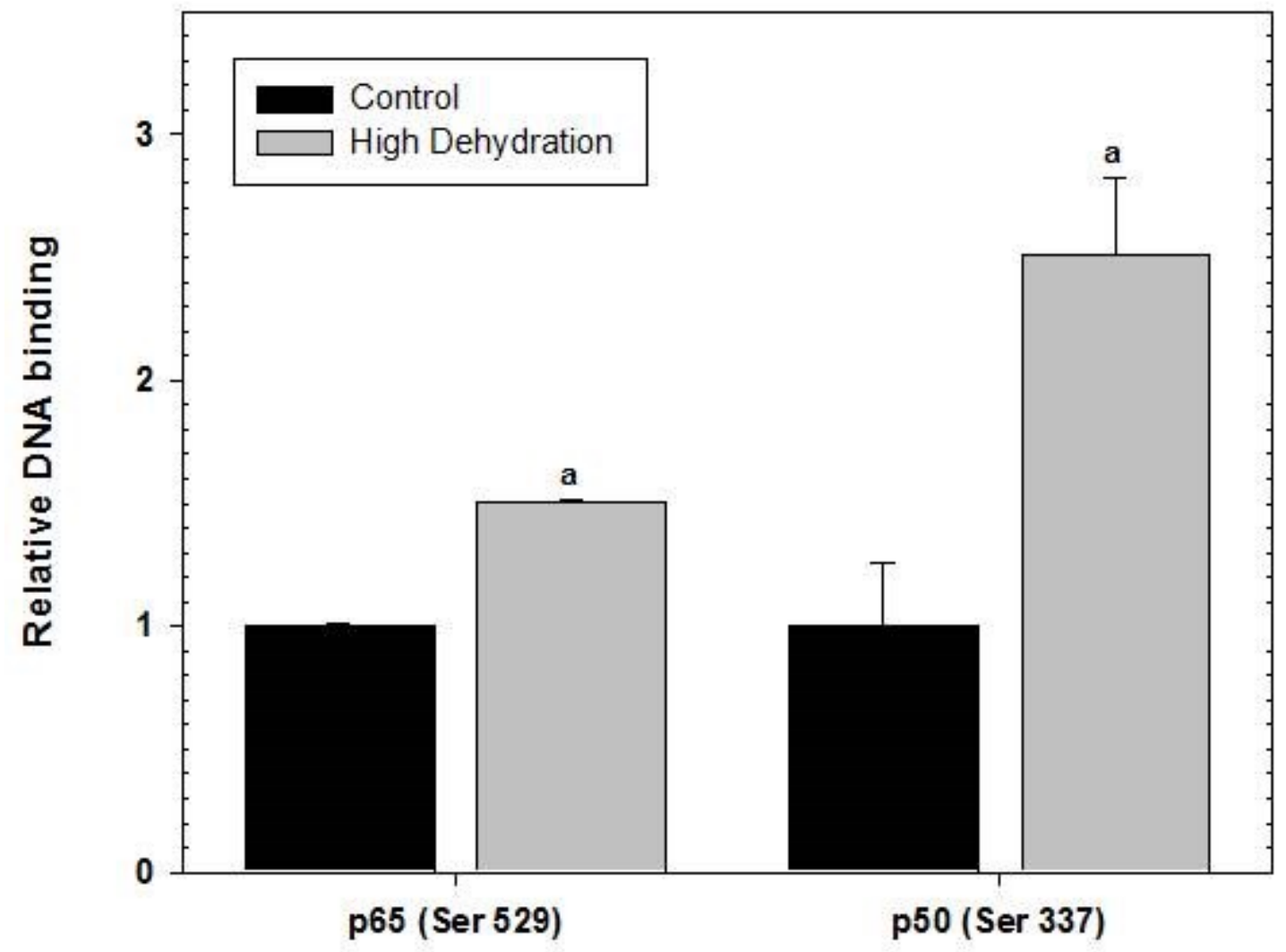

Figure 3.4: Relative changes in DNA binding of NF- $\kappa B$ subunits, p65(Ser 529) and p50 (Ser 337), compared in nuclear extracts of control and highly dehydrated $X$. laevis liver samples. Data are means \pm SEM for $\mathrm{n}=4$ independent trials. "a" - Significantly different from the control values $(P<0.05)$. 


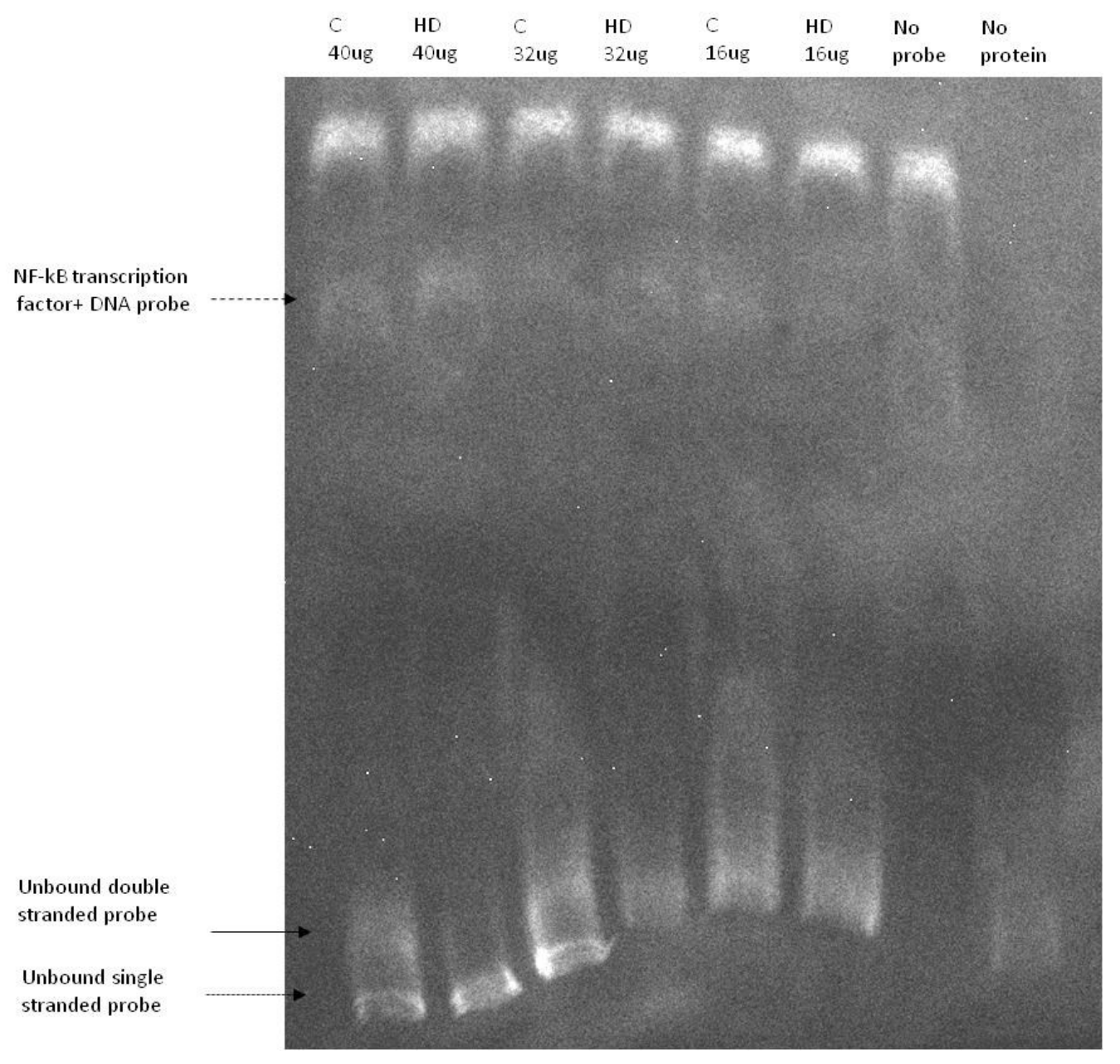

Figure 3.5: Electrophoretic Mobility Shift Assay (EMSA) gel run with different amounts $(40 \mu \mathrm{g}, 32 \mu \mathrm{g}$ or $16 \mu \mathrm{g})$ of liver nuclear protein extract from both control (C) and high dehydration (HD) conditions. The DNA-protein complex is indicated by the dashed arrow, whereas the unbound double and single stranded probe is indicated by the solid and dotted arrows, respectively. Both "no protein" and "no probe" negative controls are also included on the gel. 
A.

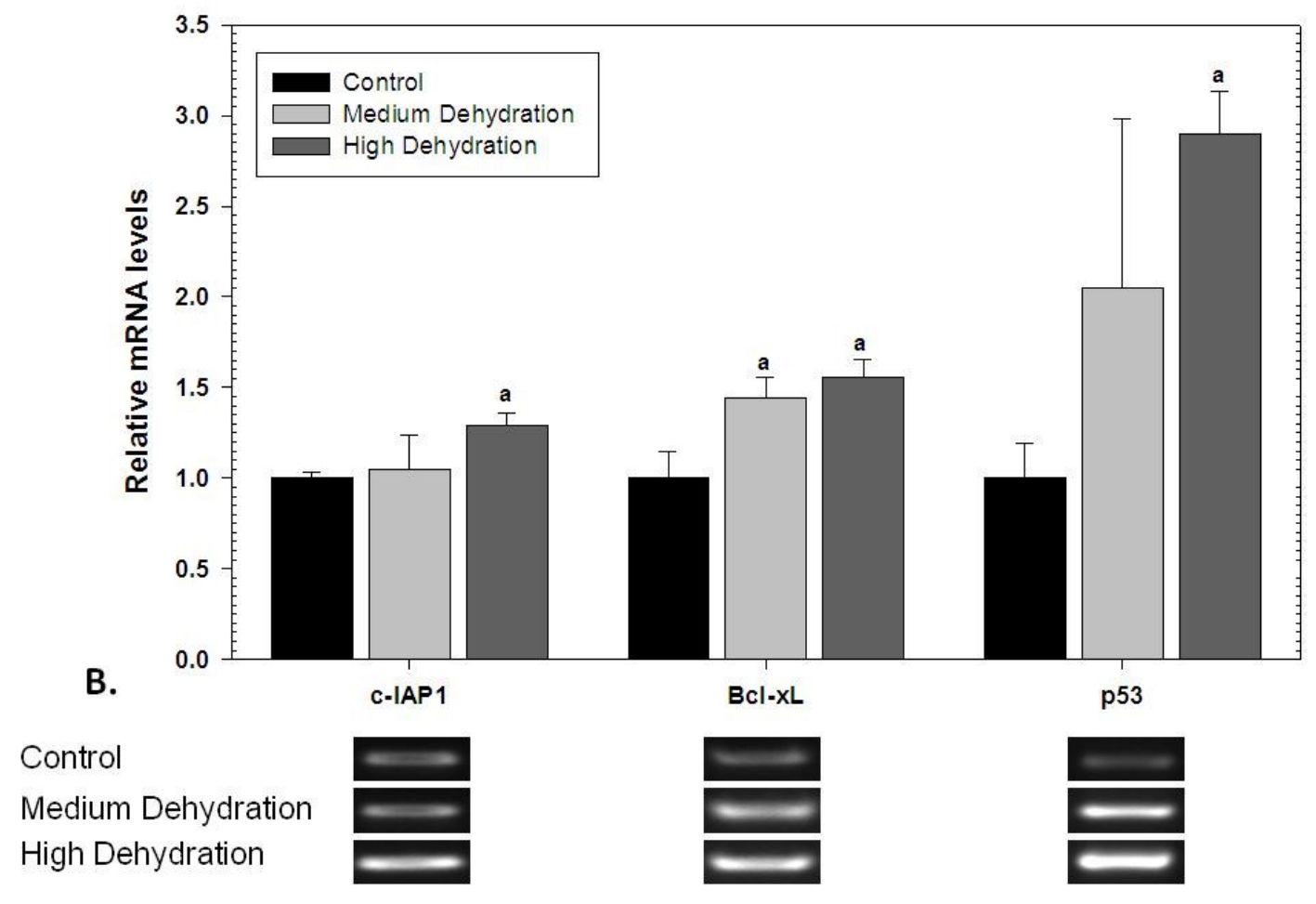

Figure 3.6: (A) Relative changes in mRNA transcript levels of NF- $\kappa B$ downstream targets; c-IAP1, Bcl-xL and p53 in liver in response to medium and high dehydration of $X$. laevis. Data are means \pm SEM for $\mathrm{n}=4$ independent trials. "a" - Significantly different from the control values $(P<0.05)$. (B) Western blots showing representative bands. 
A.

B.

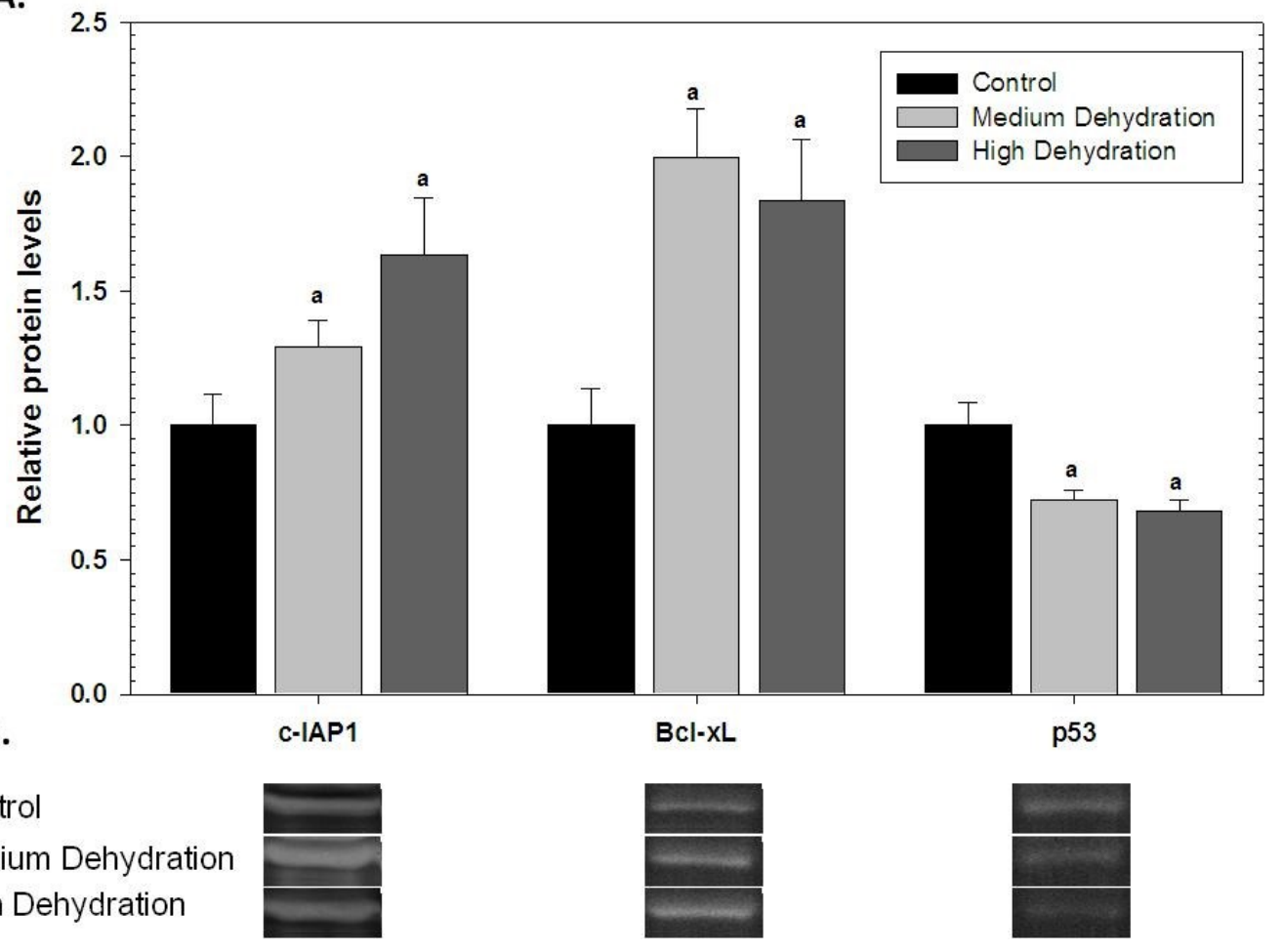

Figure 3.7: (A) Relative changes in protein levels of NF- $\kappa \mathrm{B}$ downstream targets; c-IAP1, Bcl-xL and p53 in liver tissues during medium and high dehydration of $X$. laevis. Data are means \pm SEM for $n=4$ independent trials. "a" - Significantly different from the control values $(P<0.05)$. (B) Western blots showing representative bands. 
A.

B.

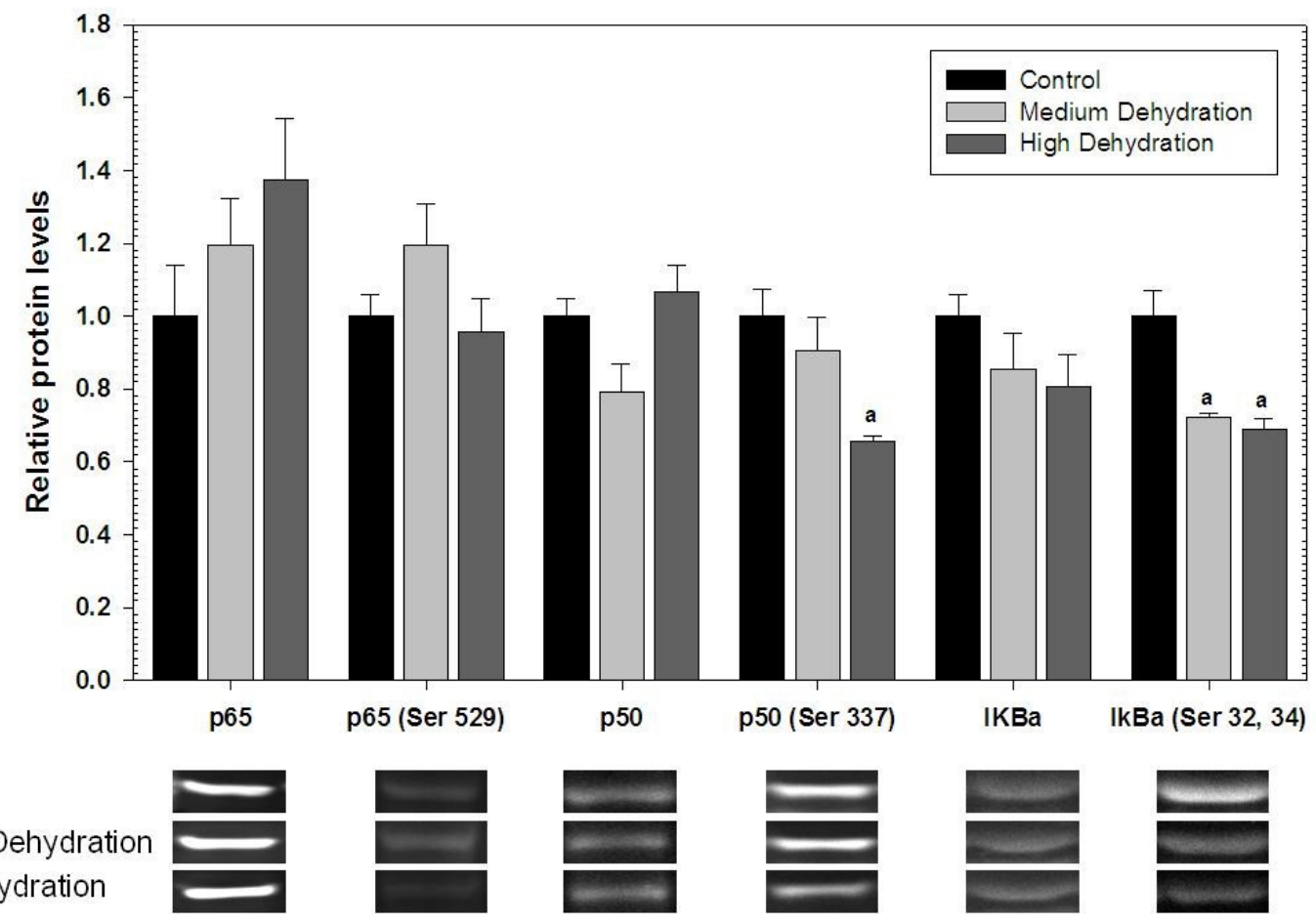

Figure 3.8: (A) Relative changes in the protein levels of NF- $\kappa \mathrm{B}$ transcription factor subunits, p50 and p65, and their phosphorylated forms, p50 (Ser 337) and p65 (Ser 529), in $X$. laevis skeletal muscle from medium and high dehydration conditions. Figure also shows the relative changes in the NF- $\kappa \mathrm{B}$ inhibitor; I $\kappa \mathrm{B} \alpha$ and its phosphorylated form $\mathrm{I} \kappa \mathrm{B} \alpha($ Ser 32, 34). Data are means \pm SEM for $\mathrm{n}=4$ independent trials. "a" - Significantly different from the control values $(P<0.05)$. (B) Western blots showing representative bands. 
A.

B.

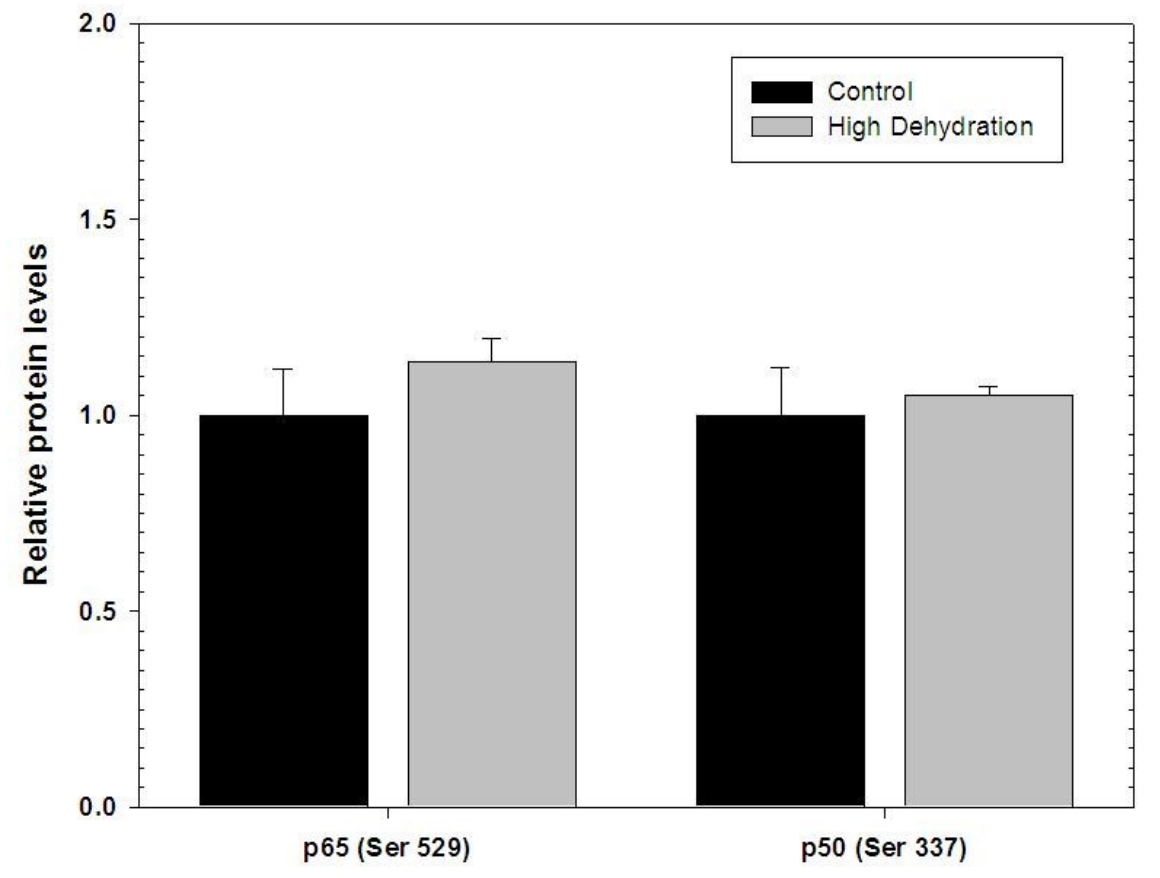

Control

(x)

High Dehydration

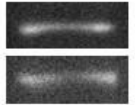

Figure 3.9: (A) Relative protein levels of p50 (Ser 337) and p65 (Ser 529) in nuclear extracts of $X$. laevis skeletal muscle from control and high dehydration conditions. Data are means \pm SEM for $\mathrm{n}=4$ independent trials. "a" - Significantly different from the control values $(P<0.05)$. (B) Western blots showing representative bands. 
A.

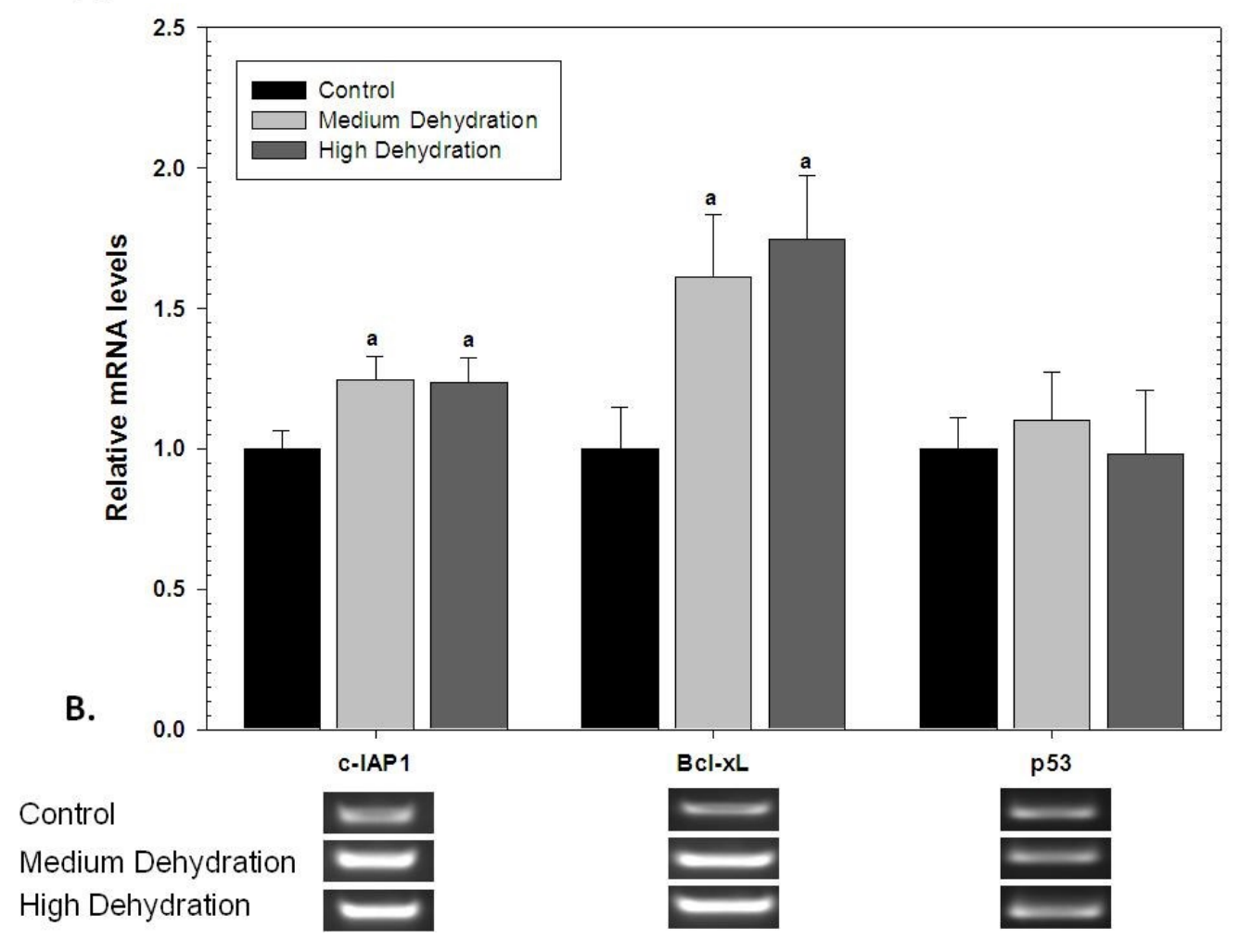

Figure 3.10: (A) Relative changes in mRNA transcript levels of NF- $\kappa B$ downstream targets; c-IAP1, Bcl-xL and p53 in skeletal muscle under medium and high dehydration of $X$. laevis. Data are means \pm SEM for $\mathrm{n}=4$ independent trials. "a" - Significantly different from the control values $(P<0.05)$. (B) Representative PCR product bands visualized on a $1 \%$ agarose gel. 
A.

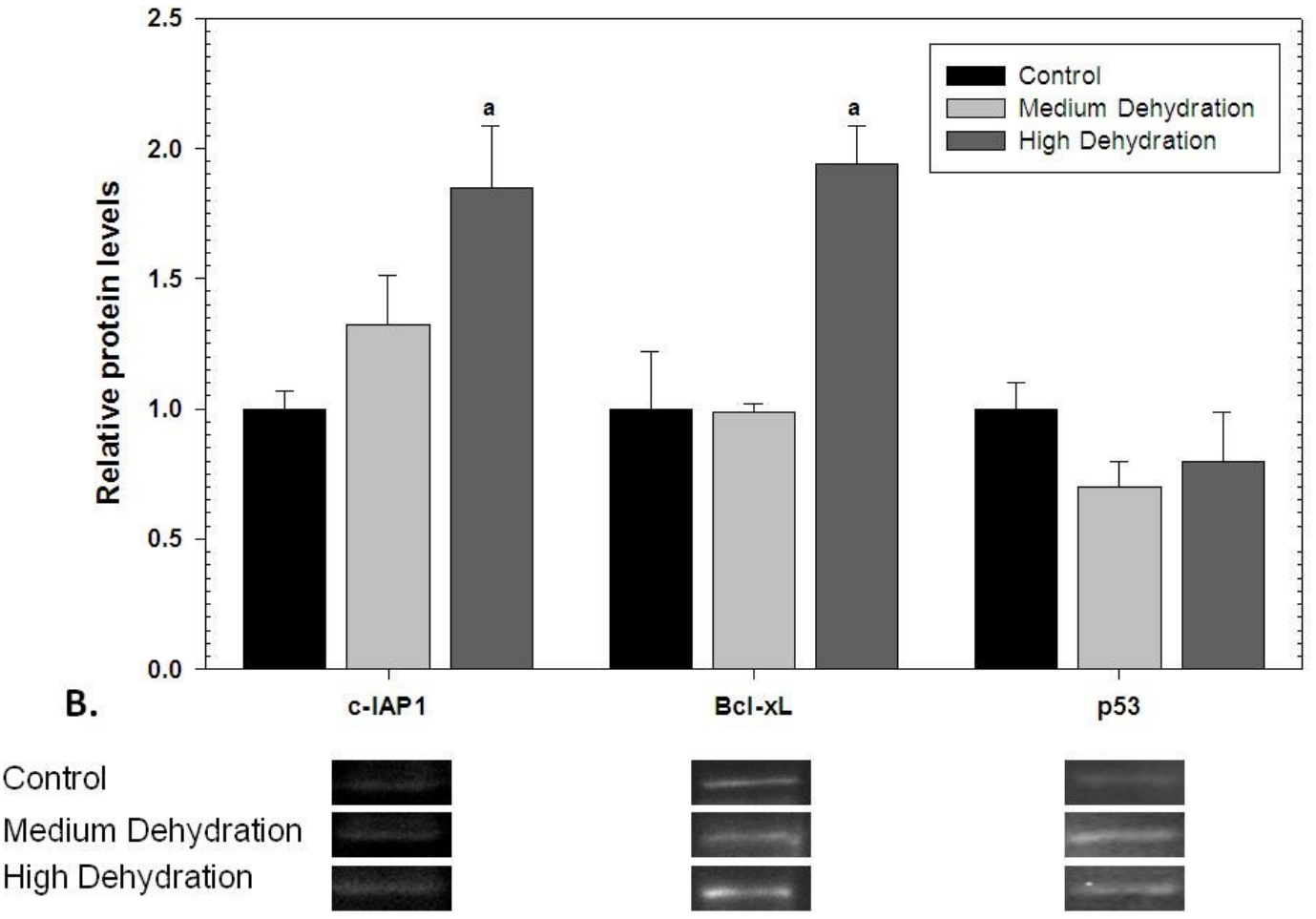

Figure 3.11: (A) Relative changes in protein levels of NF- $\kappa \mathrm{B}$ downstream targets; cIAP1, Bcl-xL and p53 in X. laevis skeletal muscle from medium and high dehydration conditions. Data are means \pm SEM for $\mathrm{n}=4$ independent trials. "a" - Significantly different from the control values $(P<0.05)$. Data are means $\pm \mathrm{SEM}$ for $\mathrm{n}=4$ independent trials. (B) Western blots showing representative bands. 


\section{References}

Adams, J.M. and S. Cory. 1998. The Bcl-2 protein family: arbiters of cell survival. Science. 281(5381): 1322-1326.

Baldwin, A.S Jr. 1996. The NF-kappa B and I kappa B proteins: new discoveries and insights. Annu. Rev. Immunol. 14: 649-681.

Basse' res, D.S. and A.S. Baldwin. 2005. Nuclear factor-kB and inhibitor of kB kinase pathways in oncogenic initiation and progression. Oncogene: 25: 6817-6830.

Biswas, D.K., Shi, Q., Baily, S., Strickland, I., Ghosh, S., Pardee, A.B. and J.D. Iglehart. 2004. NF-kappa B activation in human breast cancer specimens and its role in cell proliferation and apoptosis. Proc. Natl. Acad. Sci. USA 101(27): 10137-1042.

Bonizzi, G. and M. Karin. 2004. The two NF-kappaB activation pathways and their role in innate and adaptive immunity. Trends Immunol. 25(6): 280-288.

Borner, C. 2003. The Bcl-2 protein family: sensors and checkpoints for life-or-death decisions. Mol. Immunol. 39(11): 615-647.

Devearaux, Q.L. and J.C. Reed. 1999. IAP family proteins — suppressors of apoptosis. Genes \& Dev. 13: 239-252.

Ditzel, M. and P. Meier. 2002. IAP degradation: decisive blow or altruistic sacrifice? Trends Cell Biol. 12(10): 449-452.

Duckett, C.S., Nava, V.E., Gedrich, R.W., Clem, R.J., Van Dongen, J.L., Gilfillan, M.C., Shiels, H., Hardwick, J.M. and C.B. Thompson. 1996. A conserved family of cellular genes related to the baculovirus iap gene and encoding apoptosis inhibitors. EMBO J. 15: 2685-2689.

Fujioka, S., Sclabas, G.M., Schmidt, C., Frederick, W.A., Dong, Q.G., Abbruzzese, J.L., Evans, D.B., Baker C. and P.J. Chiao. 2003. Function of nuclear factor kappaB in pancreatic cancer metastasis. Clin. Cancer Res. 9(1): 346-354.

Gottlieb, T.M. and M. Oren. 1998. p53 and apoptosis. Sem. Cancer Biol. 8(5): 359-368

Hou, S., Guan, H. and R.P. Ricciardi. 2003. Phosphorylation of serine 337 of NF-B p50 is critical for DNA binding. J. Biol. Chem. 278(46): 45994-45998.

Levine, A.J. 1997. p53, the cellular gatekeeper for growth and division. Cell 88(3): 323331. 
Li, Y.Y and X.M. Li. 2000. The IAP family: endogenous caspase inhibitors with multiple biological activities. Cell Research. 10: 169-177.

Nishikori, M. 2005. Classical and alternative NF-kB activation pathways and their roles in lymphoid malignancies. J. Clin. Exp. Hematopathol. 45(1): 15-24.

Paul, H.L. 1999. Activators and target genes of Rel/NF- $\mathrm{BB}$ transcription factors. Oncogene 18: 6853- 6866.

Roy, N. Deveraux, Q.L., Takahashi, R., Salvesen, G.S. and J.C. Reed. 1997. The c-IAP-1 and c-IAP-2 proteins are direct inhibitors of specific caspases. EMBO J. 16(23): 69146925.

Schendel, S.L., Montal, M. and J.C. Reed. 1998. Bcl-2 family proteins as ion-channels. Cell Death Differ. 5(5): 372-380.

Senftleben, U., Cao Y., Xiao G., Greten F.R., Krähn G., Bonizzi G., Chen Y., Hu Y., Fong A., Sun S.C., and M. Karin. 2001. Activation by IKKalpha of a second, evolutionary conserved, NF-kappa B signaling pathway. Science. 293(5534): 1495-1499.

Ueda, Y. and A. Richmond. 2006. NF-kappaB activation in melanoma. Pigment Cell Res. 19(2): 112-124.

Viatour, P. Merville, M.P. Bours, V. and A. Chariot. 2005. Phosphorylation of NFkappaB and IkappaB proteins: implications in cancer and inflammation. Trends Biochem. Sci. 30(1): 43-52.

Wang, D., Westerheidge, S.D. Hanson, J.L. and Baldwin A.S. 2000. Tumor necrosis factor alpha induced phosphorylation of RelA/p65 on Ser529 is controlled by casein kinase II. J. Biol. Chem. 275(42): 32592-32597.

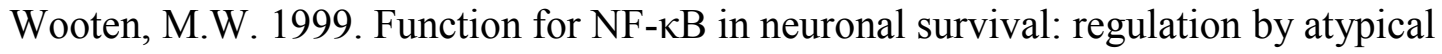
protein kinase C. J. Neurosci. Res. 58: 607-611.

Wu, H. and G. Luzano. 1994. NF-kappa B activation of p53. A potential mechanism for suppressing cell growth in response to stress. J. Biol. Chem. 269(31): 20067-20074. 


\section{Chapter 4:}

\section{Expression of the STAT transcription factors and their anti-apoptotic targets in liver and skeletal muscle of dehydrated $X$. laevis.}




\section{Introduction}

The STAT (Signal Transducers and Activators of Transcription) is a family of transcription factors that play important roles in cell growth and development (Bromberg, 2001). The family consists of seven members; STAT1 through 4, STAT5A, STAT5B and STAT6. All of the members of the family share certain functional domains. (1) The SH2 domain mediates the interaction between STATs and receptors, so that structural differences in this domain between the STAT proteins are indicative of the cytokine receptors it can bind. (2) The oligomerization domain is a structure located at the Nterminus of the STAT protein composed of eight helices with a hook-like structure. This domain is responsible for the formation of STAT tetramers or higher order oligomers. (3) The transcriptional activation domain is another domain located in the C-terminal region of STAT proteins and can undergo post-translational changes, which enhance the transcriptional ability of the STAT transcription factors (Imada and Leonard, 2000).

The STAT pathway can be activated by upwards of forty different proteins through either cytokine or G-protein receptors. In the classical activation pathway, once a ligand, such as a cytokine, binds to the appropriate receptor, the receptor dimerizes and attracts JAKs (Janus Kinases). These enzymes phosphorylate themselves and the dimerized receptor at tyrosine residues, which then provides a docking site for STAT proteins to bind by their SH2 domain (Alvarez and Frank, 2004). The JAK enzymes subsequently phosphorylate the STAT proteins at a tyrosine residue located approximately 700 residues from the N-terminus. Once activated by JAKs, STATs dissociate from the receptor and form homodimers (most commonly) or heterodimers by the binding of the phosphorylated tyrosine of one STAT to the SH2 domain of the other 
(Bromberg, 2001). The activated dimer is then translocated to the nucleus where it binds to the GAS elements in the promotor regions of its target genes and activates transcription (Imada and Leonard et al, 2000). Other activators of STATs include receptor tyrosine kinases such as EGRF (Epidermal Growth Factor Receptor) and PDGRF (Platelet Derived Growth Factor Receptor) as well as through oncogenic kinases such as Src and Abl (Alvarez and Frank, 2004).

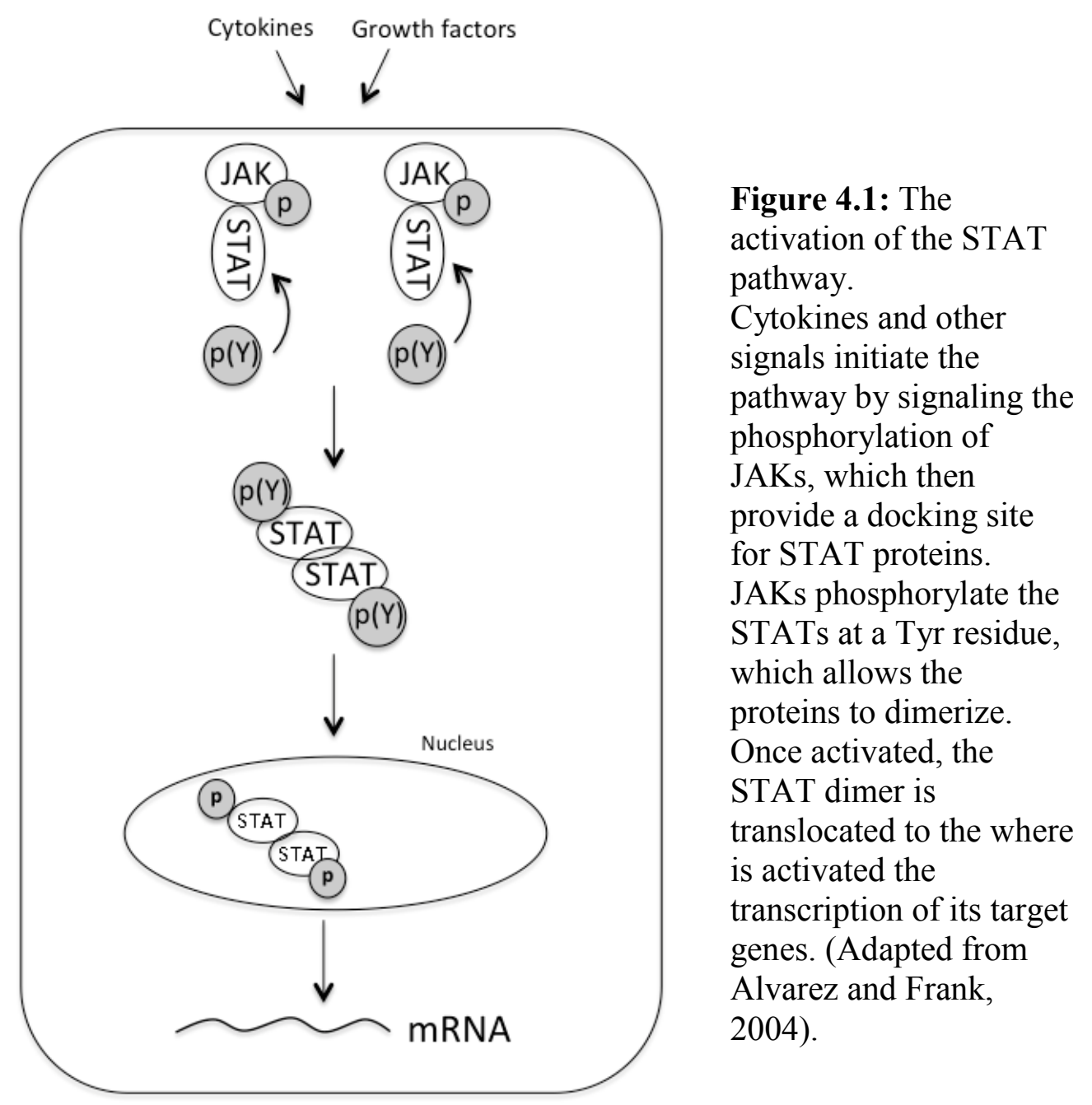


In normal cells, STAT activation lasts from several minutes to several hours in response to extrinsic stimuli. Recent findings show that in cancerous cells STAT proteins, in particular STAT1, STAT3 and STAT5, are persistently phosphorylated. Activated STAT3 has been found to be a marker of many cancers such as ovarian cancer, lung cancer, and multiple myeloma. STAT3 was also found to be elevated together with STAT5 and STAT1 in breast cancer, head and neck cancer, and AML (acute myelogenous leukemia), among others (Yu and Jove, 2004).

STAT1 and STAT2 were originally identified as components of ISGF-3 (IFN- $\alpha$ stimulated, ISRE- binding factor), a complex that is assembled in response to the presence of interferons, proteins that are released in response to pathogens (Schindler et al, 2007). Once IFN- $\alpha / \beta$ proteins activate STAT 1 and STAT2, they associate with the DNA binding protein IRF-9 (p48) to form ISGF-3, which enters the nucleus and binds to IFN-stimulated response element (ISRE) of its target genes (Park et al, 2000). ISGF-3 responsive genes include $2^{\prime}-5^{\prime}$ oligoadenylate synthetase (OAS), a protein with antiviral functions, Mx, a protein with antiviral activity against a wide range of RNA viruses, and double-stranded RNA kinase (PKR), a protein that responds to viral infection and is responsible for protein synthesis arrest under conditions of stress (Kristiansen et al, 2010; Haller and Kochs, 2002; Sadler and Williams, 2007; Park et al, 2000). STAT1 and STAT2 knockout mice are viable and display normal development but are very susceptible to viral infections and other pathological agents (Ihle, 2001).

STAT1 can also form a homodimer in response to IFN- $\gamma$ stimulation, which binds to the GAS element of IFN- $\gamma$ inducible genes and activates genes responsible for inflammation and programmed cell death (Stark, 1998). Such target genes include 
DAPK3 (death-associated protein kinase 3), a protein with functions in apoptosis and APOL6, which induces mitochondria-mediated apoptosis (Bhinge et al, 2007). To date, little is known about STAT2 homodimers, it is not clear whether these dimers are formed and if they are capable of binding DNA and independently activate transcription (Schindle et al, 2007).

The role of STAT3 has been more challenging to describe as STAT3-deficient mice result in early fetal death (Imada and Leonard et al, 2000). It has been identified to respond to signals induced by the entire IL6 and IL10 family of cytokines and found to be essential to the anti-inflammatory response (Schindle et al, 2007). Further studies have reported that STAT3 can be activated by a number of different ligands such as EGF, PDGF, CNTF, OSM, LIF as well as oncogenic signals (Bromberg, 2001). Constitutive STAT3 expression has been detected in multiple human cancers such as breast, prostate, multiple myeloma and leukemia among others (Yu and Jove, 2004). The active form of STAT3 in human cancer has been identified to mediate cellular transformation and cell cycle progression through its target genes such as matrix metalloproteinase-9 (MMP-9), a protein with function in tumor growth and metastasis, and c-myc, a transcription factor with roles in cell proliferation (Dechow et al, 2004; Alvarez and Frank, 2004). In addition, STAT3 was found to play an important role in the prevention of apoptosis in several cancers through downstream targets such as Mcl-1, Bcl-2 and Bcl-xL (Bromberg, 2001; Alvarez and Frank, 2004). More evidence for the involvement of STAT3 in cell transformation was provided by dominant-active and antisense approaches where when introduced to transformed cells were able to suppress aspects of the transformed phenotype. 
STAT4 and STAT6 are activated by IL12 and IL4 respectively and serve a function in the immune response through the activation of IL12 and IL4 inducible genes, especially those responsible for $\mathrm{T}$ helper cell differentiation. CD4+ T helper lymphocytes are able to differentiate into two separate subsets. T helper 1 (TH1) cells produce IL-2 and IFN $\gamma$ and defend against infections and cancer. T helper 2 (TH2) cells produce IL4, IL5, IL6 and IL13 and responsible for humoral immunity and the protection against parasitic antigens (Wurster et al, 2000). STAT4 deficient cells are unresponsive to IL12 and suffer from impaired TH1 cell development (Imada and Leonard et al, 2000). A number of STAT4 downstream target genes have been identified to date but the exact mechanism by which STAT4 causes Th1 differentiation is still unclear. Examples of such targets are ERM and IFN-inducible factor-1 (IRF-1), both transcription factors induced by IL12 binding (Wurster et al, 2000). STAT6 deficient mice also result in defective T cell differentiation where the cell is unable to respond to IL4 binding and produce Th2 cells (Imada and Leonard et al, 2000). STAT6 downstream targets include MHC class II, CD23, and IL-4R $\alpha$, all IL4 inducible genes that play a role in immune defense (Wurster et al, 2000).

STAT5A and STAT5B share $96 \%$ amino acid identity and have been shown to posses both redundant and non-redundant functions (Schindler et al, 2007). STAT5A deficient mice studies have presented evidence for the role of this protein in lactation, where STAT5A-null mice have impaired lobuloalveolar proliferation of the mammary epithelium during pregnancy. STAT5B null mice resulted in impaired growth hormone signaling causing a disruption in the fetal differentiation into a male or female (Imada and Leonard, 2000). Both STAT5A and B deficient mice show a decrease in natural killer 
(NK) cell development and STAT5A/B double deficient mice have no NK cells. Studies with STAT5A/B deficient mice have also demonstrated that these transcription factors also become activated in response to IL2 and IL7, erythropoietin (EPO) and thrombopoietin (TPO) receptor signaling (Ihle, 2001). Once phosphorylated by JAKs these two transcription factors can form both homodimers $(5 \mathrm{a} / 5 \mathrm{a})$ and heterodimers $(5 \mathrm{a} / 5 \mathrm{~b})$ and translocate to the nucleus where they activate the transcription of their target genes. These transcription factors have not been found to dimerizes with less related STAT family members (Grimleya et al, 1999).

Of particular interest is the function of STAT5 activation in the regulation of antiapoptotic genes such as $b c l-x l$. Studies have demonstrated that the $b c l$-xl contains a STAT5 binding site and in recent years it has been found that this transcription factor is involved in anti-apoptotic signaling during fetal liver erythropoiesis (Socolovsky et al. 1999).

STAT3 and STAT5 have been found to play important roles in cell survival and proliferation. These two proteins are the two members of the STAT family that are most frequently associated with human cancers because their downstream targets are essential for tumor survival and development (Alvarez and Frank, 2004). STAT3 downstream targets include members of the Bcl-2 family, such as Bcl-2, Bcl-xL, and Mcl-1, members of the inhibitors of apoptosis (IAP) family, such as c-IAP1/2 and survivin, as well as pim1/2 (Alvarez and Frank, 2004; Lanuti et al, 2009). STAT5 contributes to cell survival by also activating the transcription of proteins such as Bcl-2, Bcl-xL, Mcl-1, pim-1 as well as XIAP (Alvarez and Frank, 2004; Yu and Jove, 2004; Mohapatra et al, 2003). 
Several studies using dominant-negative STAT3 have reported an increased apoptotic response in tumor cells such as multiple myeloma, breast cancer, mycosis fungoides, lung cancer, prostate cancer, and melanoma Alvarez and Frank, 2004). These and other findings constitute the base work for gene therapy research using dominantnegative STAT3 and STAT5 to induce the death response in tumor cells (Yu and Jove, 2004).

This chapter focuses on the expression profile of STAT3 and STAT5 in dehydrated Xenopus liver and skeletal muscle tissue. The activation of anti-apoptotic pathways under the stress condition is examined through the regulation of downstream targets Bcl-2 and Mcl-1.

Bcl-2 and Mcl-1 are both members of the Bcl-2 family of proteins, which is central in the regulation of the cellular death response as it consists of both pro-apoptotic and anti-apoptotic signals. The family includes 15 family members each of which contains 3 or 4 conserved motifs known as Bcl-2 homology domains (BH1- BH4). These domains do not have any enzymatic activity but have functions in protein-protein interaction (Adams and Cory, 1998). This related group of proteins contains both antiapoptotic (Bcl-2, Bcl-xL, Mcl-1 etc) and pro-apoptotic members (Bax, BAD, Bak etc). The higher relative expression levels of one group compared to the other determine whether the cell is destined to survive or not (Borner, 2003).

The anti-apoptotic members of the Bcl-2 family are localized to the outer mitochondrial membrane, while Bcl-2 itself is also found on the nuclear membrane and the endoplasmic reticulum (Kroemer, 1997). Following a death signal, the pro-apoptotic members, localized to the cytosol or cytoskeleton, undergo a conformational change to 
enable them to integrate into membranes such as the mitochondrial membrane. One mode of action of the Bcl-2 family is to guard the mitochondrial membrane by opposing the action of the anti-apoptotic proteins (Gross et al, 1999). For example, it has been shown that activated Bcl-2 protein targets Raf-1 to the mitochondria, where it mediates the phosphorylation and inactivation of Bad, a pro-apoptotic member of the Bcl-2 family. Bcl-2 localized to the endoplasmic reticulum has been shown to directly bind the apoptotic protein Bax preventing it from executing its detrimental functions on the mitochondria (Kroemer, 1997).

The pro-apoptotic members of the Bcl-2 family bind to the mitochondrial membrane and induce the release of over 30 different proteins into the cytoplasm. One of these proteins is cytochrome $\mathrm{c}$ which binds apoptotic protease activating factor 1 (Apaf1) along with dATP to form an apoptosome. This complex proceeds to activate caspase-9 which cleaves caspase- 3 to its active form and allow it to carry out its apoptotic functions (Borner, 2003). Other proteins that are released from the mitochondria include Endonucleus $\mathrm{G}$ which is an enzyme that enters the nucleus and degrades genomic DNA, thus initiating the cell death response in a caspase independent manner (Borner, 2003). Thus Bcl-2 prevents the onset of apoptosis by targeting its pro-apoptotic family members and preventing the release of destructive proteins from the mitochondria (Kroemer, 1997).

The second downstream target that was investigated in this chapter is myeloid cell leukaemia-1 (Mcl-1), a protein that also belongs to the Bcl-2 family of anti-apoptotic proteins. The details on the mechanism by which Mcl-1 exerts its anti-apoptotic function are still unknown but research suggests that it is able to bind and neutralize pro-apoptotic 
proteins Bim and Bak. By binding these proteins, Mcl-1 prevents the release of cytochrome $\mathrm{c}$ from the mitochondria along with other destructive proteins. It has been found that, unlike Bcl-2, expression of Mcl-1 is downregulated during apoptosis potentially due to cleavage by caspases, demonstrating that Mcl-1 inhibition is required for the initiation of the cell death response (Michaels et al, 2005).

This chapter evaluates the expression levels of STAT1-3 along with STAT5 and STAT6 during $X$. laevis dehydration in two tissues, liver and skeletal muscle, through the examination of relative abundance of the STAT proteins in total and nuclear extracts along with a study of their DNA binding ability. In addition, two anti-apoptotic downstream targets, Bcl-2 and Mcl-1, are examined by measuring their relative transcript and protein levels under stress conditions compared to controls.

\section{Results}

\section{Relative expression levels of STAT proteins in liver}

Relative protein levels of six STAT proteins were compared in control, medium dehydration (MD) and high dehydration (HD) conditions in the liver tissue of Xenopus frogs (figure 4.2). Each of STAT1 (Tyr 701), STAT2 (Tyr 690), STAT3 (Ser 727), STAT3 (Tyr 705), STAT5 (Tyr 694) and STAT6 (Tyr 641) cross reacted with a single band at $\sim 91,113,86,86,90$ and $110 \mathrm{kDa}$ respectively. Relative levels of STAT1 (Tyr 701), STAT2 (Tyr 690) and STAT6 (Tyr 641) remained the same across all conditions. Expression levels of STAT3 (Ser 727) and STAT3 (Tyr 705) significantly increased in both MD and HD compared to controls $(P<0.05)$. STAT3 (Ser 727) protein levels were found to be elevated to $1.35 \pm 0.11$ in medium dehydration and further elevated to $1.65 \pm$ 0.07 in high dehydration, while STAT3 (Tyr 705) increased to $2.05 \pm 0.16$ and $1.77 \pm$ 
0.07 in medium and high dehydration, respectively compared to controls. STAT5 (Tyr 694) protein levels significantly decreased in high dehydration to $43 \% \pm 6 \%$ of control.

Analysis of nuclear translocation of STAT3 (Ser727) and STAT3 (Tyr705) subunits in liver

As STAT3 (Ser727) and STAT3 (Tyr705) total protein levels were found to be significantly increased in dehydrated frogs, nuclear extracts from control and highly dehydrated Xenopus frogs were analyzed to assess whether these proteins have also translocated into the nucleus (Figure 4.3). A single band cross reacted with STAT3 (Ser727) and STAT3 (Tyr705) at about $86 \mathrm{kDa}$ in both proteins. Relative protein levels of STAT3 (Ser727) significantly increased to $1.53 \pm 0.15$ while relative protein levels of STAT3 (Tyr705) dropped to just $7 \% \pm 2 \%$, in highly dehydrated frogs compared to controls $(P<0.05)$.

\section{Analyzing DNA-binding of STAT3 (Tyr705) in liver}

The relative amount of DNA-binding by STAT3 in nuclear extracts of liver was measured using an ELISA procedure. Figure 4.4 shows that relative binding of STAT3 (Tyr 705) was significantly increased under high dehydration conditions, levels rising by 1.6-fold compared to control values $(P<0.05)$. Increases in relative DNA binding are indicative of a significant increase in the amount of STAT3 in the in the nucleus of liver cells under dehydrated conditions. 


\section{EMSA validation}

In order to validate the results obtained in the DNA binding experiments, an EMSA procedure was used to determine if $X$. laevis STAT bound specifically to the designed probe representing the consensus DNA binding element. Samples of liver nuclear extracts were incubated with double-stranded DNA probe and DNA-protein complexes were allowed to form and then separated on a polyacrylamide gel. Figure 4.5 shows an EMSA image of a gel run from incubations with $20 \mu \mathrm{g}$ of liver nuclear extract from control versus HD conditions, along with a "no protein" and "no probe" controls.

The image shows a band corresponding to the STAT/DNA complex that does not appear in the two control lanes along with a band at the bottom of each lane corresponding to the unbound probe. The unbound single stranded probe and unbound double stranded probe failed to separate. These results suggest that there was a shift from the low molecular weight unbound probe to the higher molecular weight protein/DNA complex. Since the probe was designed to bind both STAT3 and STAT5 the results apply to both of these proteins and did not need to be repeated in the later experiments.

\section{Analysis of STAT3 downstream targets' transcript levels in liver}

Two downstream targets of STAT3, Bcl-2 and Mcl-1, were analyzed using RTPCR in Xenopus liver tissue (Figure 4.6). Relative transcript levels of $b c l-2$ did not differ across conditions while transcript levels of $m c l-1$ significantly decreased in both medium and high dehydration compared to controls $(P<0.05)$. $m c l-1$ transcript levels decreased to $42 \% \pm 4 \%$ and $33 \% \pm 2 \%$ of control values in MD and $\mathrm{HD}$, respectively. 


\section{Analysis of STAT3 downstream targets' protein levels in liver}

Relative protein levels of the downstreams, Bcl-2 and Mcl-1, were analyzed by immunoblotting in Xenopus liver tissue (Figure 4.7). Two post-translational modifications of Bcl-2, Bcl-2 (Thr56) and Bcl-2 (Ser70), were analyzed in addition to total protein levels. Total Bcl-2 antibody crossreacted with a single band at $\sim 26 \mathrm{kDa}$ while the two phosphorylated forms crossreacted at $28 \mathrm{kDa}$. The Mcl-1 antibody produced a single band at $\sim 40 \mathrm{kDa}$. Relative protein levels of total Bcl-2, Bcl-2 (Thr56) and Mcl-1 did not change across conditions. Bcl-2 (Ser70) appeared to increase only in medium dehydration to $1.5 \pm 0.07$ compared to control values $(P<0.05)$.

\section{Relative expression levels of STAT proteins in skeletal muscle}

Relative expression levels of STAT1 (Tyr 701), STAT2 (Tyr 690), STAT3 (Ser 727), STAT3 (Tyr 705), STAT5 (Tyr 694) and STAT6 (Tyr 641) were analyzed in Xenopus skeletal muscle tissue (Figure 4.8). Relative protein levels of STAT2 (Tyr 690), STAT3 (Tyr 705), and STAT6 (Tyr 641) did not change across conditions. STAT1 (Tyr 701 ) protein levels significantly decreased to $57 \% \pm 13 \%$ of control values in medium dehydration while STAT3 (Ser 727) significantly decreased to $51 \% \pm 8 \%$ of control values in high dehydration $(P<0.05)$. STAT5 (Tyr 694) relative protein levels increased significantly to $3.2 \pm 0.24$ in the medium dehydration condition compared to control levels $(P<0.05)$.

\section{Analysis of nuclear translocation of STAT5 (Tyr 694) in skeletal muscle}

Relative protein levels of STAT5 (Tyr 694) were analyzed in nuclear extracts of skeletal muscle in Xenopus (Figure 4.9). STAT5 (Tyr 694) relative expression levels 
significantly increased to $1.77 \pm 0.09$ in medium dehydration and $1.93 \pm 0.19$ in high dehydration compared to controls $(P<0.05)$.

\section{Analysis of DNA-binding of STAT5 (Tyr 694) in skeletal muscle}

DNA-binding ability of STAT5 (Tyr 694) in skeletal muscle tissue in Xenopus frogs was assessed using an ELISA technique (Figure 4.10). Relative DNA-binding was observed in both experimental conditions however significant changes were only found for the high dehydration conditions. STAT5 (Tyr 694) DNA- binding affinity increased to $1.58 \pm 0.16$ in $\mathrm{HD}$ compared to control values $(P<0.05)$.

\section{Analysis of STAT5 downstream targets' transcript levels in skeletal muscle}

Figure 4.11 shows the relative transcript levels of STAT5 downstream targets, $b c l-2$ and $m c l-1$, assessed using RT-PCR in X. laevis skeletal muscle from control, MD and HD conditions. Under both dehydration conditions $b c l-2$ transcript levels increased significantly, by $3.47 \pm 0.29$ fold in MD and 3.4 fold \pm 0.1 in $\mathrm{HD}$, compared to controls $(P<0.05)$. Transcript levels of $m c l-1$ increased significantly only in the HD condition, rising by $1.4 \pm 0.1$ fold, compared to controls $(P<0.05)$.

\section{Analysis of STAT5 downstream targets' protein levels in skeletal muscle}

Xenopus liver extracts were analyzed by immunoblotting for relative changes in the protein levels of total Bcl-2, Bcl-2 (Thr 56), Bcl-2 (Ser 70) and Mcl-1 proteins in response to whole body dehydration (Figure 4.12). Total Bcl-2 antibody crossreacted with a single band at $\sim 26 \mathrm{kDa}$ while the two phosphorylated forms cross reacted at $\sim 28$ 
$\mathrm{kDa}$. The Mcl-1 antibody produced a single band at $40 \mathrm{kDa}$. Relative levels of total Bcl2 protein significantly increased in both experimental conditions, rising to $1.58 \pm 0.11$ fold in MD and $1.54 \pm 0.12$ fold in HD, compared to controls $(P<0.05)$. Bcl-2 (Thr 56) protein levels decreased significantly in MD, to $63 \pm 10 \%$ of controls, and continues to decrease in HD, falling to just $42 \pm 4 \%$ of controls $(P<0.05)$. Opposite results were seen in the relative levels of Bcl-2 (Ser 70) where protein levels significantly increased in MD and HD, by $1.66 \pm 0.06$ fold and $1.85 \pm 0.34$ fold, respectively compared to controls $(P<0.05)$. No significant changes were found in relative protein levels of Mcl-1 between conditions.

\section{Discussion}

The present chapter evaluated the activation of the STAT transcription factor pathway and their anti-apoptotic targets during the stress of dehydration in X. laevis liver and skeletal muscle. The STAT transcription factors play important roles in the immune response, T helper cell differentiation, cell cycle progression, in addition to cell growth and survival.

The family consists of seven members: STAT1 STAT2, STAT3, STAT4, STAT5A, STAT5B and STAT6 (Imada and Leonard, 2000). Under conditions of stress, the pathway is initiated by the phosphorylation of JAK enzymes, which then proceed to phosphorylate STATs at a tyrosine residue (Alvarez and Frank, 2004). The phosphorylated STATs form either homodimers or heterodimers by binding of the phosphorylated tyrosine of one STAT to the SH2 domain of the other (Bromberg, 2001). 
The activated dimer is translocated to the nucleus where it binds to the promotor region of its target genes to initiate their transcription (Alvarez and Frank, 2004).

The two main STAT proteins that activate anti-apoptotic genes in response to a stimulus are STAT3 and STAT5. These were found to be differentially regulated in the liver and skeletal muscle tissue of dehydrated Xenopus frogs. STAT3 contains two main phosphorylation sites; (1) tyrosine 705, required for STAT3 activation and translocation into the nucleus and (2) serine 727, required for maximal transcription ability. Wen et al (1995) found that a STAT3 transcription factor that is phosphorylated at tyrosine 705 but contains a mutation on serine 727 is able to bind DNA but the rate of transcription is significantly reduced, by as much as $60 \%$ (Wen et al, 1995). Both phosphorylated forms were found to be significantly elevated in both dehydration conditions compared to controls in Xenopus liver tissue. STAT3 (Tyr 705) was found to be significantly increased by 2 -fold and 1.8-fold in medium and high dehydration, respectively compared to controls while STAT3 (Ser 727) was found to be elevated to 1.4-fold in medium dehydration and then further elevated to 1.7-fold in high dehydration compared to controls and medium dehydration. STAT5 (Tyr 694) protein levels were found to be significantly decreased in the high dehydration condition when compared to medium dehydration and controls. The relative expression levels of STAT1 (Tyr 701), STAT2 (Tyr 690) and STAT6 (Tyr 641) were found to remain constant between all conditions.

Further evidence for the activity of the STAT3 transcription factor during Xenopus dehydration was provided by examining the relative protein levels in nuclear extract as well as data provided by DNA binding experiments (Figures 4.3 and 4.4). Relative protein levels of STAT3 (Tyr705) significantly increased by 1.5 -fold in nuclear 
extracts of the high dehydration condition in the liver tissue while relative levels of STAT3 (Ser727) dropped to just 7\% of controls in the high dehydration condition. These data provide evidence for the translocation of the activated STAT3 protein into the nucleus but do not explain why STAT3 phosphorylated at serine 727 does not appear to have also moved from the cytoplasm into the nucleus. As previously stated only phosphorylation at a tyrosine residue is required for STAT3 activation and DNA-binding (Wen et al, 1995). However, the reason behind the absence of STAT3 (Ser727) in the nucleus despite the elevation in total protein extracts requires further investigation.

Furthermore, DNA binding activity of STAT3 (Tyr705) was significantly enhanced in liver under high dehydration. This was evident from ELISA analyses where relative DNA binding has showed an increase of 1.6-fold in high dehydration compared to controls. These data provide strong evidence for the increased transcriptional activity of STAT3 during the stress of dehydration.

The skeletal muscle tissue did not show evidence for STAT3 activation as relative protein levels of STAT3 (Tyr 705) remained unchanged between conditions and levels of STAT3 (Ser 727) significantly decreased during high dehydration in total extracts. However, relative levels of STAT5 (Tyr 694) did significantly differ from controls, rising by 3.2-fold in the medium dehydration condition compared to controls. Surprisingly, relative protein levels of STAT5 (Tyr 694) were found to be significantly elevated in both medium and high dehydration conditions compared to controls in the nuclear extracts of the skeletal muscle tissue. To provide further evidence for STAT5 activation in both dehydration conditions, changes in DNA-binding were analyzed in both MD and HD 
Xenopus skeletal muscle. Results showed that DNA binding activity was significantly enhanced in high dehydration, increasing by 1.8 -fold compared to controls.

In summary, relative protein levels of STAT5 were elevated only in medium dehydration in total protein extracts but significantly increased in both medium and high dehydration in nuclear extracts. Data from DNA-binding studies showed a significant increase in high dehydration but not in medium dehydration. Taken together, the data presents strong evidence for the increased transcriptional activity of STAT5 in the high dehydration condition while the degree of STAT5 activation in medium dehydration is not as clear.

Relative levels of other STATs did not present evidence for their activation in the skeletal muscle tissue during dehydration. STAT2 and STAT6 protein levels remained unchanged while levels of STAT1 decreased significantly in the medium dehydration condition.

The three STAT proteins that are most relevant to the study of the programed cell death response are STAT1, STAT3 and STAT5. In response to a death stimulus, STAT1 forms a homodimer and translocates into the nucleus where it activates the transcription of apoptotic genes such as DAPK3 (death-associated protein kinase 3) and APOL6, which induces mitochondria-mediated apoptosis (Bhinge et al, 2007). In this study, relative levels of STAT1 were constant across conditions in the liver tissue and decreased significantly in medium dehydration in the skeletal muscle tissue. These results suggest that this apoptotic member of the STAT family is not activated during dehydration in Xenopus. 
STAT3 and STAT5 were found to be differentially regulated in the liver and skeletal muscle tissue during dehydration stress. In liver, STAT3 appeared to be activated and translocated to the nucleus while the same was true for STAT5 in the skeletal muscle tissue. Once activated, both STAT3 and STAT5 enter the nucleus and promote the transcription of vital genes such as those responsible to resisting apoptotic signals. They share a number of downstream targets two of which belong to the Bcl-2 family of proteins; Bcl-2 and Mcl-1. The cause for the preferred activation of one STAT over the other in a specific tissue is unclear. Further studies investigating the upstream and downstream players in the STAT pathway may provide an explanation for these results.

The Bcl-2 family includes 15 family members each of which contains 3 or 4 conserved motifs known as Bcl-2 homology domains (BH1- BH4). The family consists of both anti-apoptotic (ex. Bcl-2 and Mcl-1) and pro-apoptotic members (ex. Bax and $\mathrm{Bad})$. Bcl-2 is localized to the mitochondrial membrane as well as the nuclear membrane and the endoplasmic reticulum. Following the activation of the cell death program, Bcl-2 targets a protein kinase named Raf-1 to the mitochondrial membrane. Bound Raf-1 protein mediates the phosphorylation and inactivation of Bad, preventing it from initiating apoptosis (Wang et al, 1996).

The anti-apoptotic function of Bcl-2 is dependent on its phosphorylation status. It has been shown that kinases such as p38 mitogen-activated protein kinases (p38 MAPK) mediate Bcl-2 phosphorylation at threonine 56 and serine 87 causing a decrease in its anti-apoptotic potential (De Chiara et al, 2006). Evidence shows that substitution of both Thr 56 and Ser 87 with an alanine residue inhibits cytochrome c release from isolated mitochondria after the addition of activated p38 MAPK (Chiara et al, 2005). On the other 
hand, Takahiko et al (1997) found that phosphorylation at Ser 70 is required for Bcl-2 death suppressor signaling activity. The phosphorylation of this site is most likely mediated by protein kinase $\mathrm{C}$ after the onset of a death signal (Takahiko et al, 1997).

In the present study, relative transcript levels of $b c l-2$ remained unchanged in the liver tissue across all conditions while significant increases, of over 3-fold, were found in both medium and high dehydration condition in the skeletal muscle tissue compared to controls (figure 4.6 and 4.11). Total protein levels of Bcl-2 matched the mRNA levels, as relative protein levels remained unchanged in the liver tissue while significant increases were found in both medium and high dehydration conditions in skeletal muscle (figure 4.7 and 4.12). In the liver tissue protein levels of Bcl-2 ( $\mathrm{Thr} 56$ ) remained unchanged across conditions while protein levels of the anti-apoptotic form Bcl-2 (Ser 70) were found to be significantly increased in medium dehydration. In skeletal muscle, relative protein levels of Bcl-2 (56) were significantly decreased in the medium dehydration condition compared to controls and further decreased in high dehydration compared to medium dehydration and controls. Relative protein levels of Bcl-2 (Ser 70) however, were found to be significantly elevated in both medium and high dehydration compared to controls in dehydrated Xenopus skeletal muscle.

It appears that there is a tissue dependent activation of the anti-apoptotic protein, Bcl-2. STAT3 was found to be activated in the liver tissue but does not appear to increase the transcription of Bcl-2 in this tissue as transcript and protein levels remained unchanged. There is however an indication for a STAT-independent activation of Bcl-2 in the medium dehydration condition of the liver tissue, as the anti-apoptotic form of Bcl2, Bcl-2 (Ser 70), was found to be significantly more abundant in this condition compared 
to control animals. It seems that the acute response to the stress of dehydration in the liver tissue involves the activation of $\mathrm{Bcl}-2$ through by post-translational modifications. Skeletal muscle tissue showed a different response to dehydration stress. The evidence suggest that STAT5 is activated in response to the stress of dehydration and promotes the transcription of $\mathrm{Bcl}-2$ as shown through elevated relative transcript and protein levels in both medium and high dehydration conditions. Furthermore, the proapoptotic Bcl-2 (Thr56) was found to be decreased in both dehydration conditions while the pro-survival Bcl-2 (Ser 70) was increased in both conditions. Bcl-2 appears to play a significant role in the protection against apoptosis in both tissues under conditions of stress but the evidence suggest that it is only activated in a STAT dependent manner in the skeletal muscle tissue.

Mcl-1 is another anti-apoptotic member of the Bcl-2 family and a downstream target of both STAT3 and STAT5. Further studies are required to identify its antiapoptotic functions, however it has been reported that it is able to bind and neutralize proapoptotic proteins Bim and Bak (Michaels et al, 2005). In this study, it was found that transcript levels of $m c l-1$ were significantly reduced in both conditions in the liver tissue. Relative protein levels of Mcl-1 were found to be unchanged between conditions. In skeletal muscle levels of $m c l-1$ were found to increase by 1.4 -fold in the high dehydration condition compared to controls. The conclusions from analyzing the relative protein levels of Mcl-1 were similar to the liver tissue, no changes were found between all conditions investigated.

It is unclear why transcript levels of $m c l-1$ were elevated in high dehydration in skeletal muscle while protein levels remained unchanged. One explanation may be that 
those transcripts are being stored in the nucleus or cytoplasm in preparation for rehydration. Studies with other torpid animal models have found that cells are able to store mRNA transcripts during dormancy by the binding of nuclear bodies and cytoplasmic p-bodies. In order to preserve energy during dormancy, protein synthesis is limited but these transcripts remain available for rapid onset of protein synthesis during the exit from torpor. It is possible that Mcl-1 is actively transcribed and stored until the alleviation of the stress conditions (Storey, 2010).

Overall, this chapter provided evidence for the activation of the anti-apoptotic STAT pathway in both liver and skeletal muscle tissues of dehydrated $X$. laevis. The liver tissue showed evidence for the transcriptional activity of STAT3 while skeletal muscle showed evidence for the transcriptional activity of STAT5. In addition, it was found that Bcl-2, an anti-apoptotic downstream of STAT5, was actively transcribed in the skeletal muscle tissue and activated by phosphorylation at serine 70 . It is clear that although differences were found between the two tissues with respect to STAT activation, both tissues displayed evidence of anti-apoptotic activity during dehydration. 


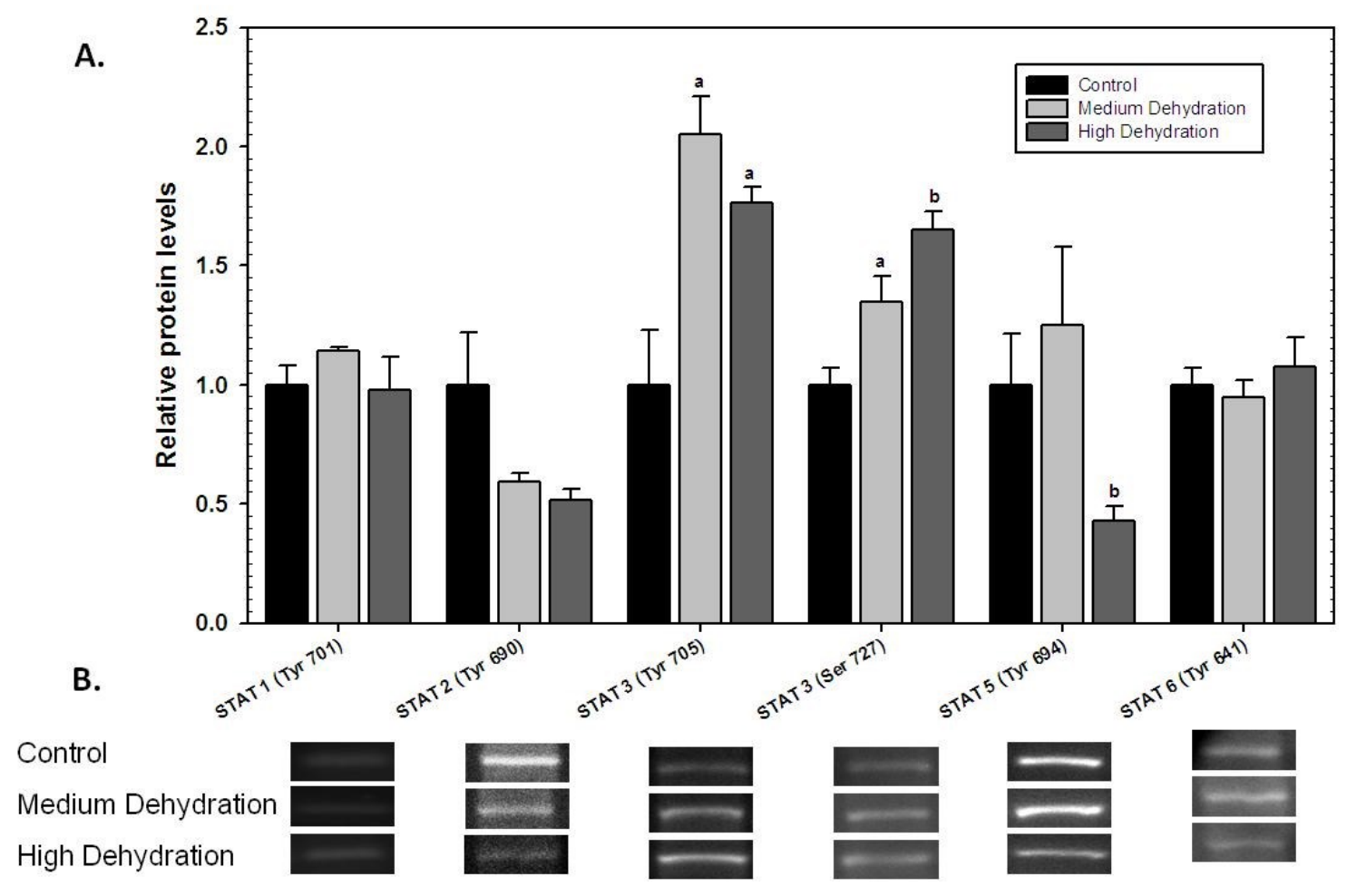

Figure 4.2: (A) Relative changes in protein expression of phosphorylated STAT transcription factors, STAT1 (Tyr 701), STAT2 (Tyr 690), STAT3 (Tyr 705), STAT3 (Ser 727), STAT5 (TYR 694) and STAT6 (Tyr 641), in liver tissue during medium and high dehydration of $X$. laevis. Data are means \pm SEM, $n=4$ independent trials on tissue samples from different animals. "a" - Significantly different from the control values; "b" - significantly different from medium dehydration, $(P<0.05)$. (B) Western blots showing representative bands. 
A.

B.

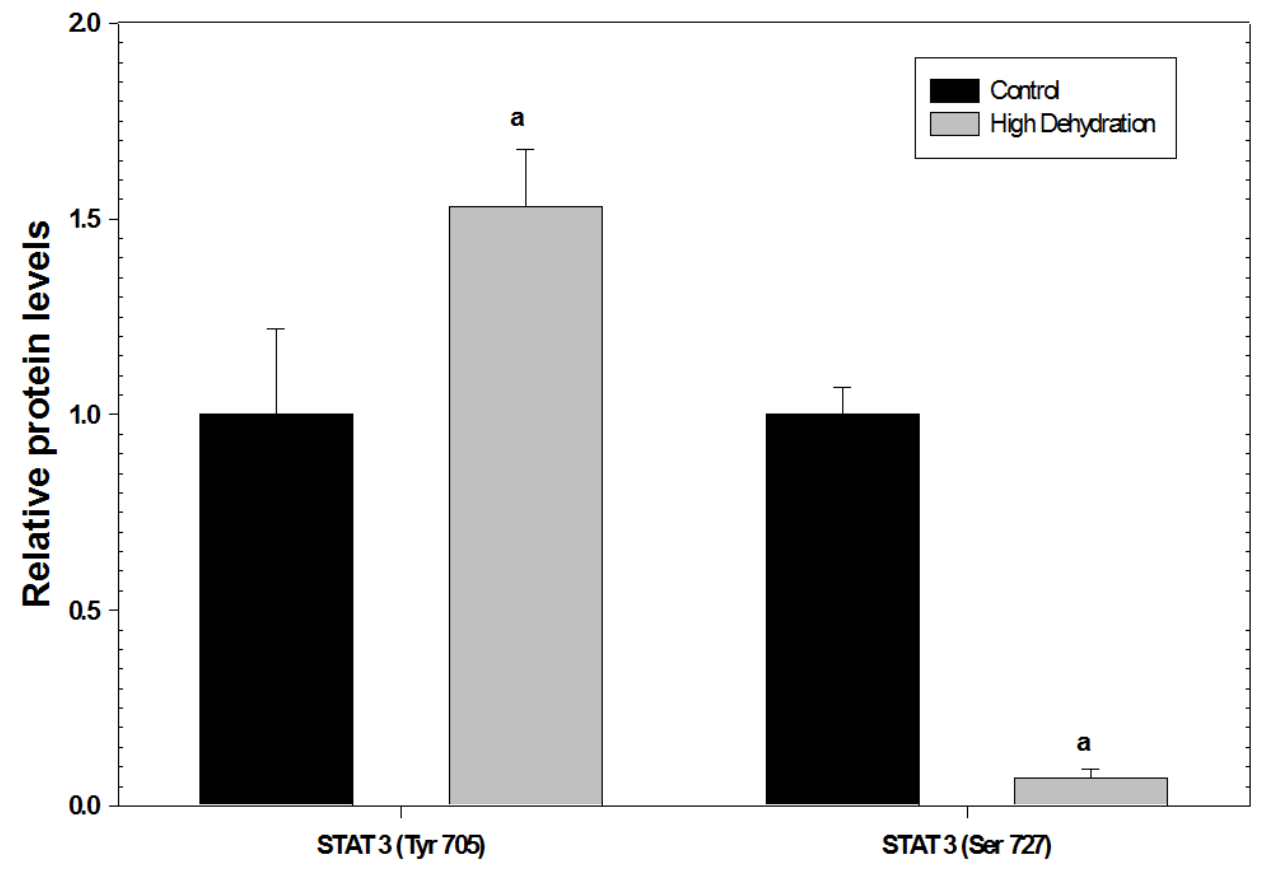

Control

High Dehydration
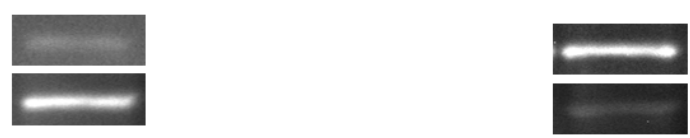

Figure 4.3: (A) Relative protein levels of STAT3 (Tyr 705) and STAT3 (Ser 727) in nuclear extracts of liver from control and high dehydration X. laevis. Data are means \pm SEM for $\mathrm{n}=4$ independent trials on tissue samples from different animals. " $\mathrm{a}$ " Significantly different from the control values $(P<0.05)$. (B) Western blots showing representative bands. 


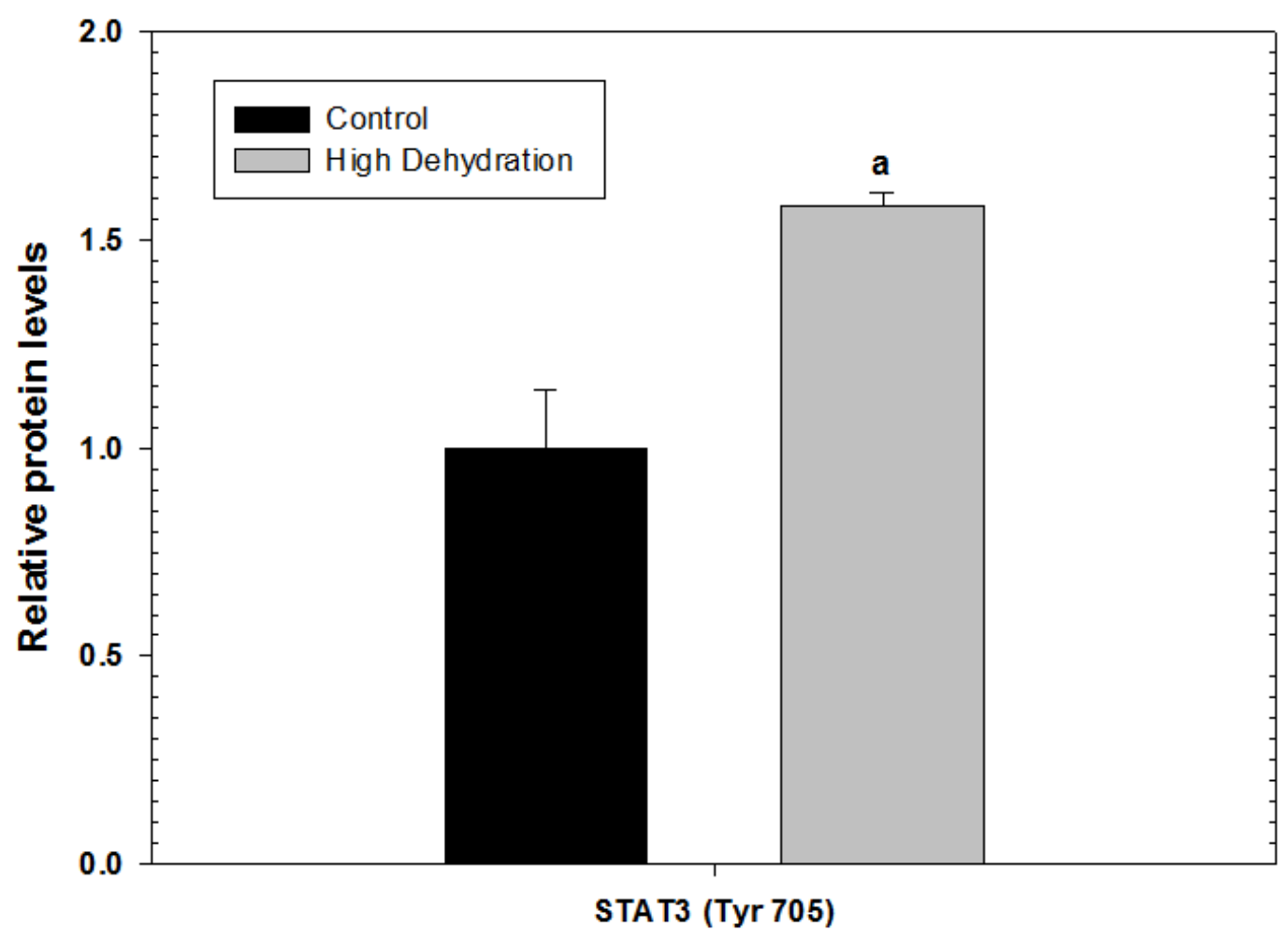

Figure 4.4: Relative changes in DNA binding of STAT3 (Tyr 705) compared in nuclear extracts of control and highly dehydrated $X$. laevis liver samples. Data are means \pm SEM for $\mathrm{n}=4$ independent trials. "a" - Significantly different from the control values $(P<0.05)$. 


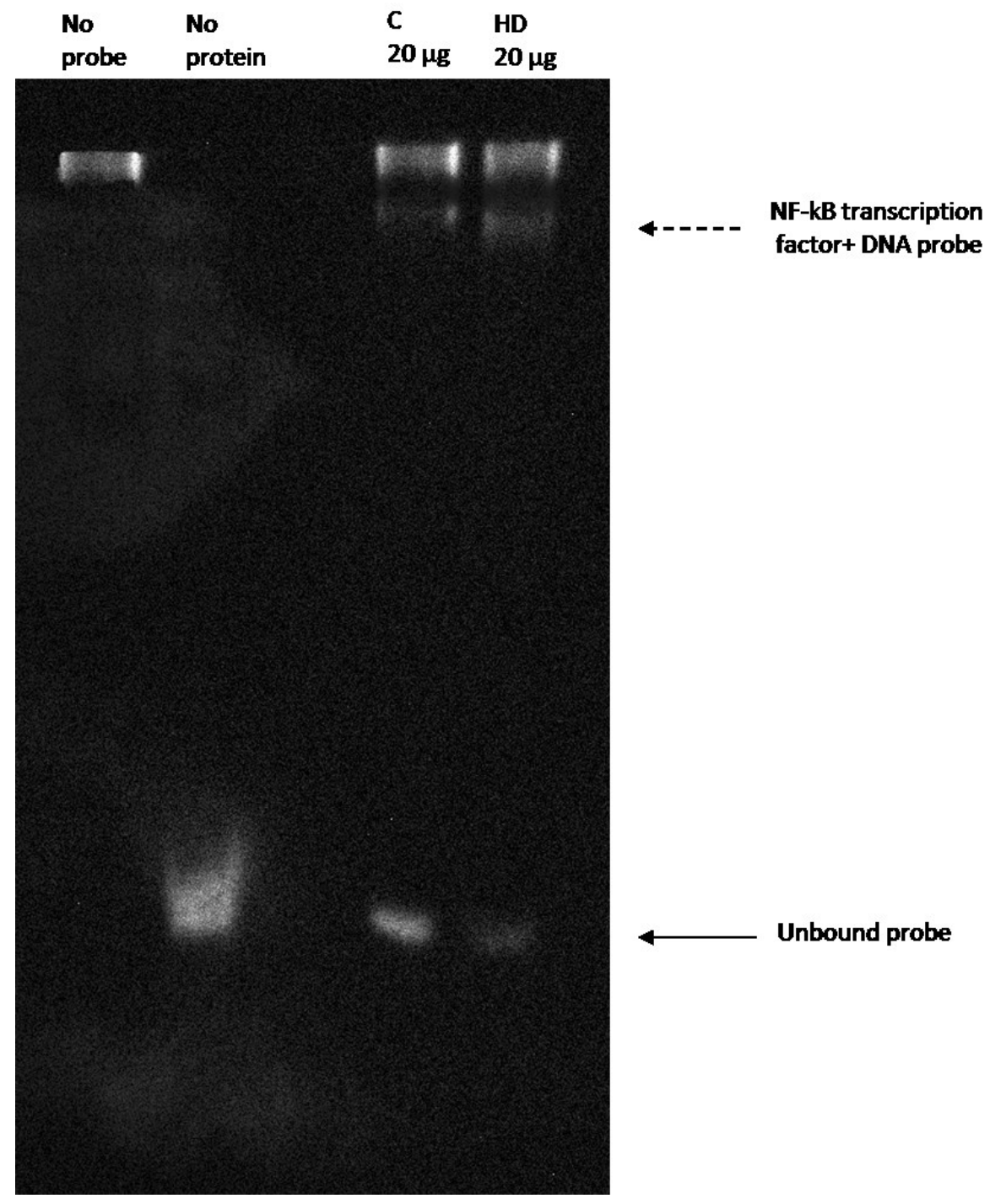

Figure 4.5: Electrophoretic Mobility Shift Assay (EMSA) gel of liver nuclear protein extract from both control (C) and high dehydration (HD) conditions. The DNA-protein complex is indicated by the dashed arrow, whereas the unbound probe is indicated by the solid arrow. Both "no protein" and "no probe" negative controls are also included on the gel. 
A.

B.
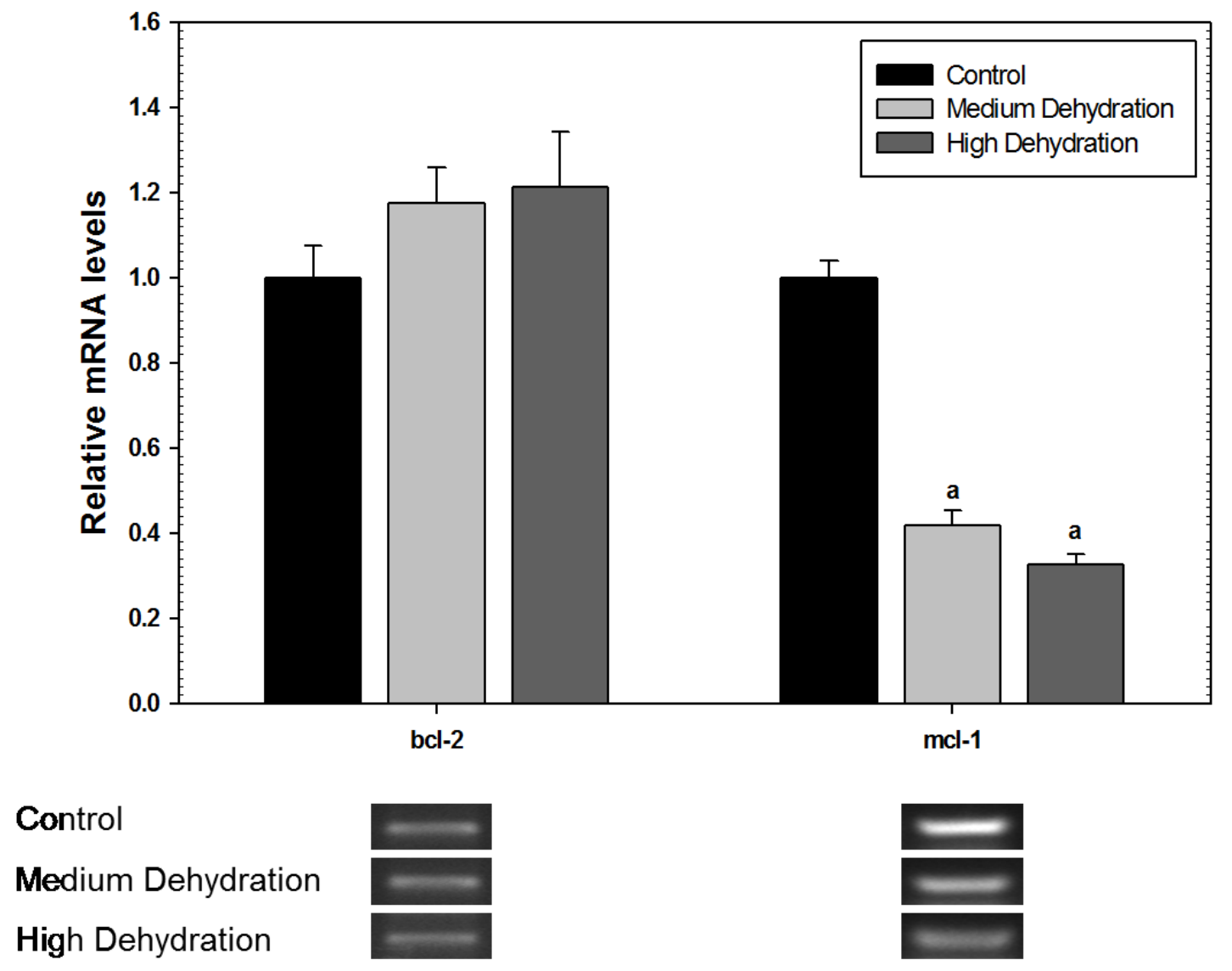

Figure 4.6: (A) Relative changes in mRNA transcript levels of STAT3 downstream targets; bcl-2 and mcl-1 in liver in response to medium and high dehydration of X. laevis. Data are means \pm SEM for $\mathrm{n}=4$ independent trials. "a" - Significantly different from the control values $(P<0.05)$. (B) Representative PCR product bands visualized on a $1 \%$ agarose gel. 


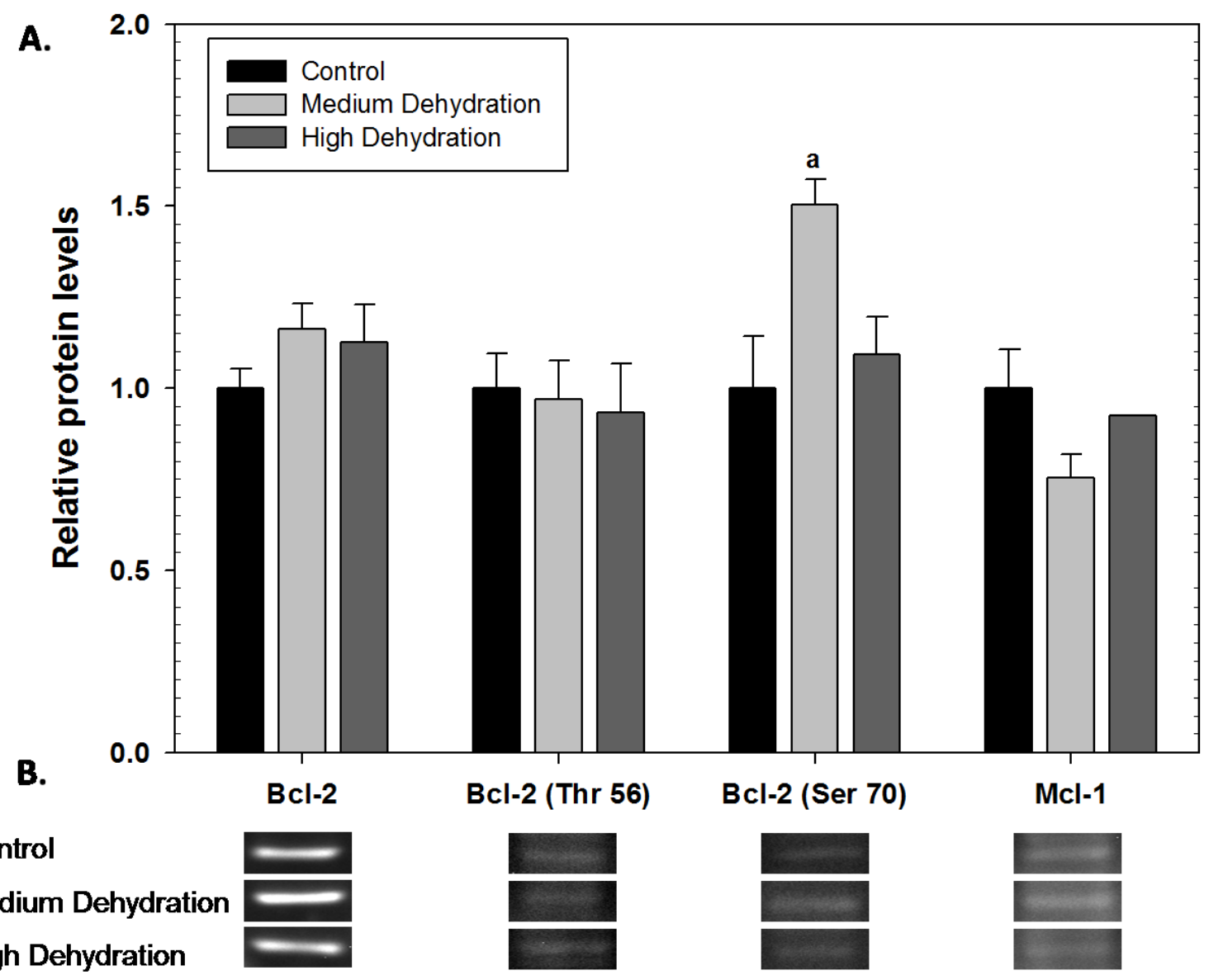

Figure 4.7: (A) Relative changes in protein levels of STAT3 downstream targets; total bcl-2 in addition to bcl-2 (Thr 56) and bcl-2 (Ser 70) in liver tissues during medium and high dehydration of $X$. laevis. Data are means \pm SEM for $\mathrm{n}=4$ independent trials. "a" Significantly different from the control values $(P<0.05)$. (B) Western blots showing representative bands. 
A.

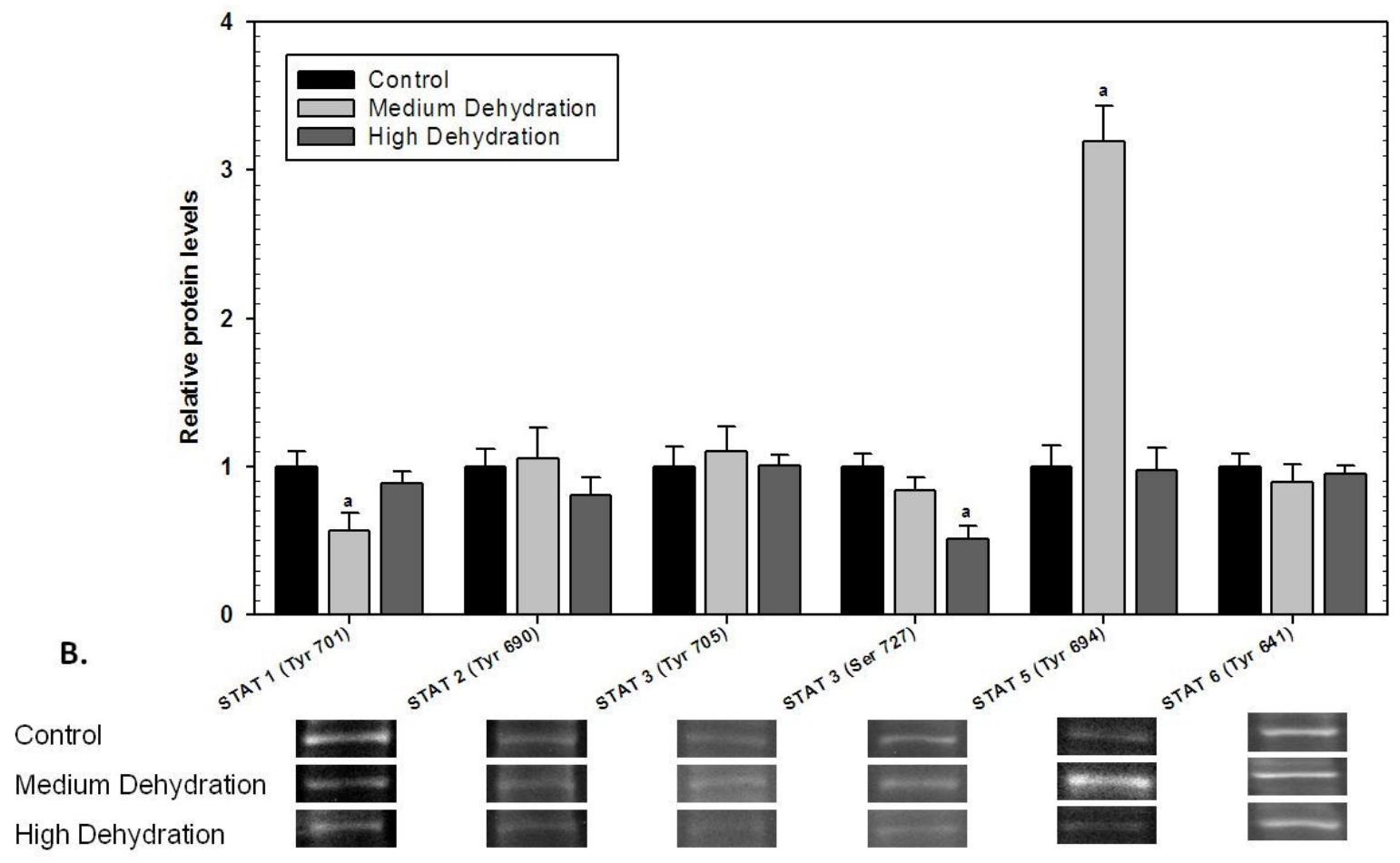

Figure 4.8: (A) Relative changes in the protein levels of STAT transcription factors, STAT1 (Tyr 701), STAT2 (Tyr 690), STAT3 (Tyr 705), STAT3 (Ser 727), STAT5 (TYR 694) and STAT6 (Tyr 641), in X. laevis skeletal muscle from medium and high dehydration conditions. Data are means \pm SEM for $n=4$ independent trials. "a" Significantly different from the control values $(P<0.05)$. (B) Western blots showing representative bands. 


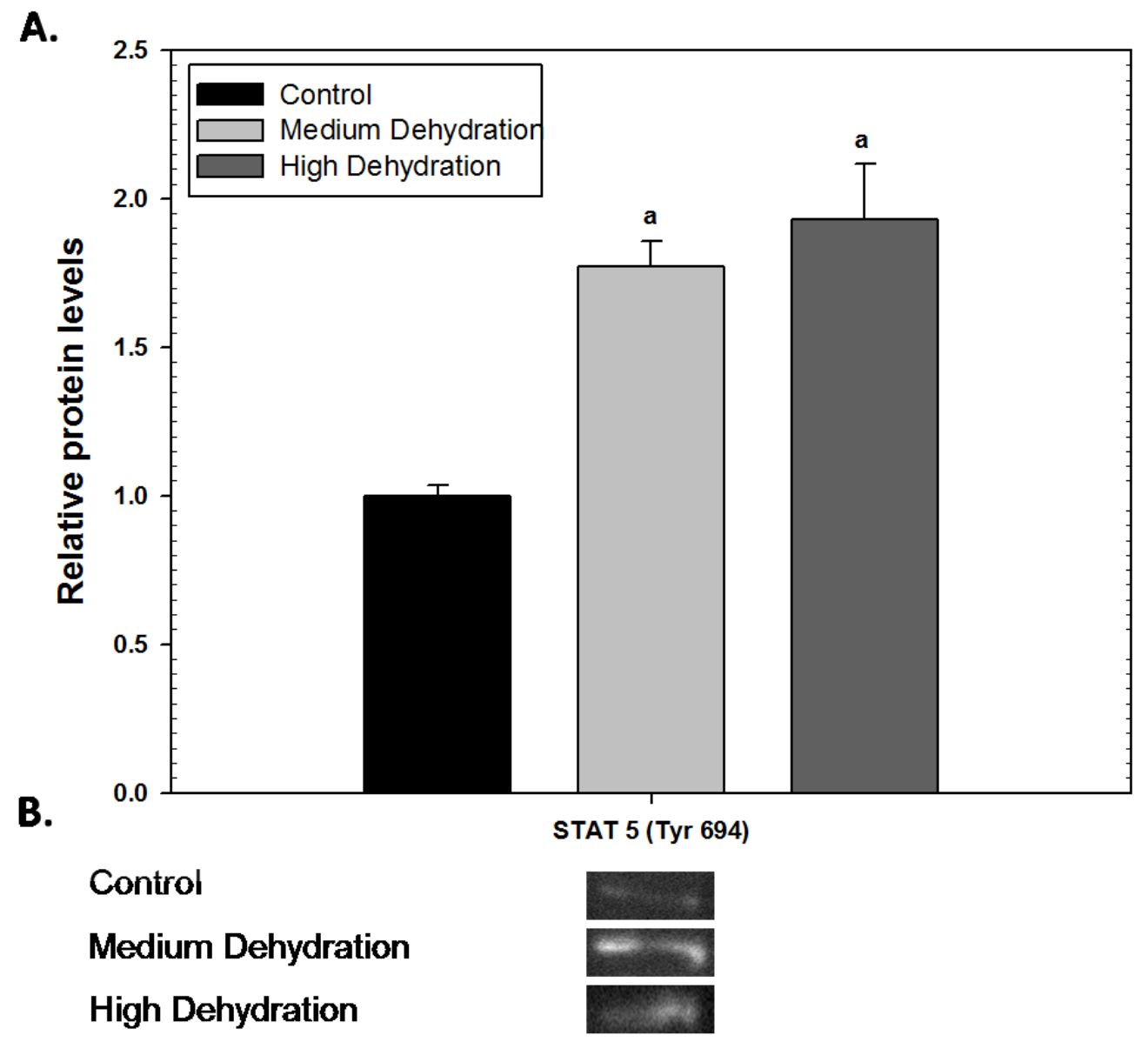

Figure 4.9: (A) Relative protein levels of STAT5 (Tyr 694) in nuclear extracts of liver from control, medium dehydration, and high dehydration X. laevis. Data are means \pm SEM for $n=4$ independent trials on tissue samples from different animals. "a" Significantly different from the control values $(P<0.05)$. (B) Western blots showing representative bands. 


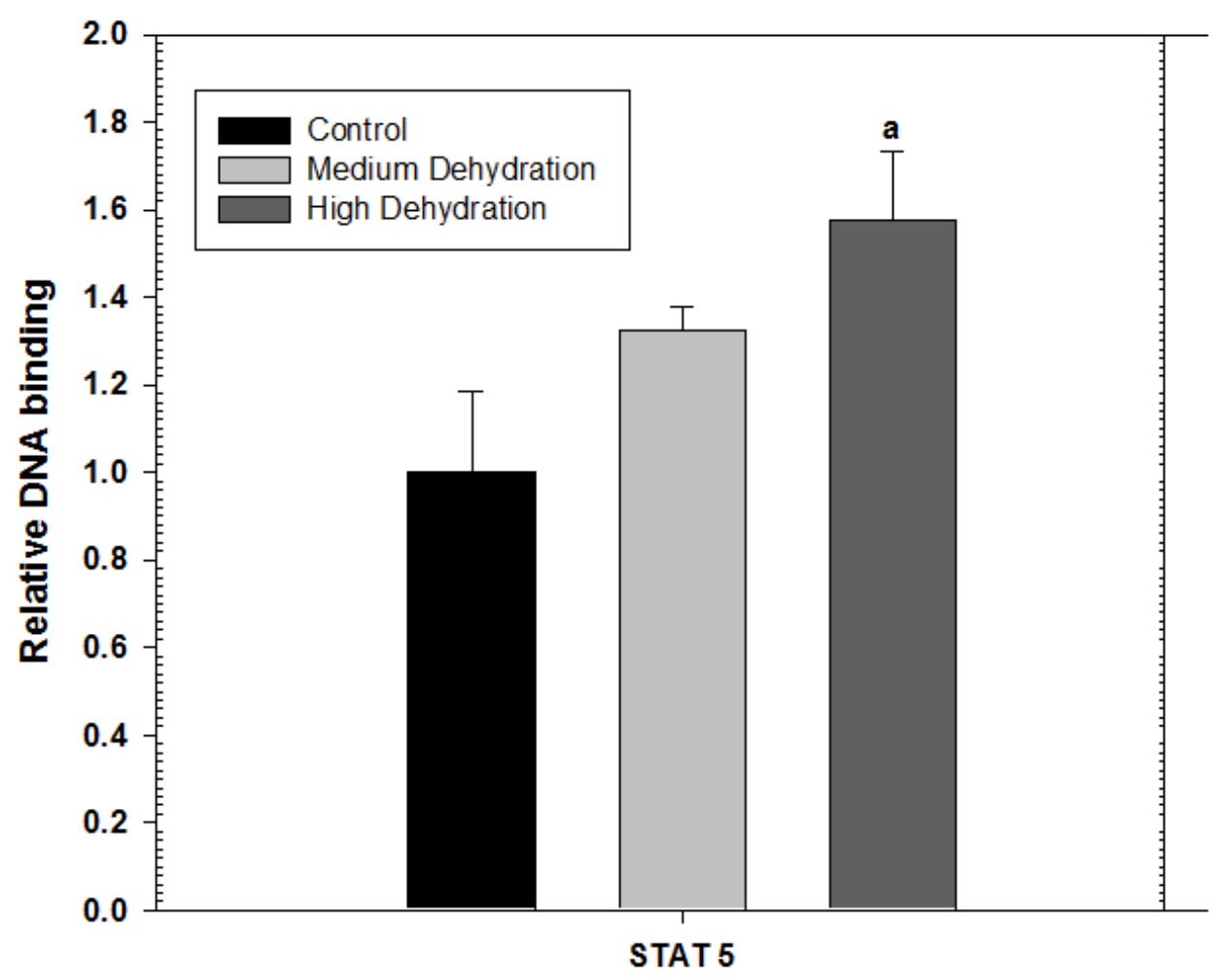

Figure 4.10: Relative changes in DNA binding of STAT5 (Tyr 694) compared in nuclear extracts of control, medium dehydrated, and highly dehydrated $X$. laevis muscle samples. Data are means \pm SEM for $n=4$ independent trials. "a" - Significantly different from the control values $(P<0.05)$. 
A.

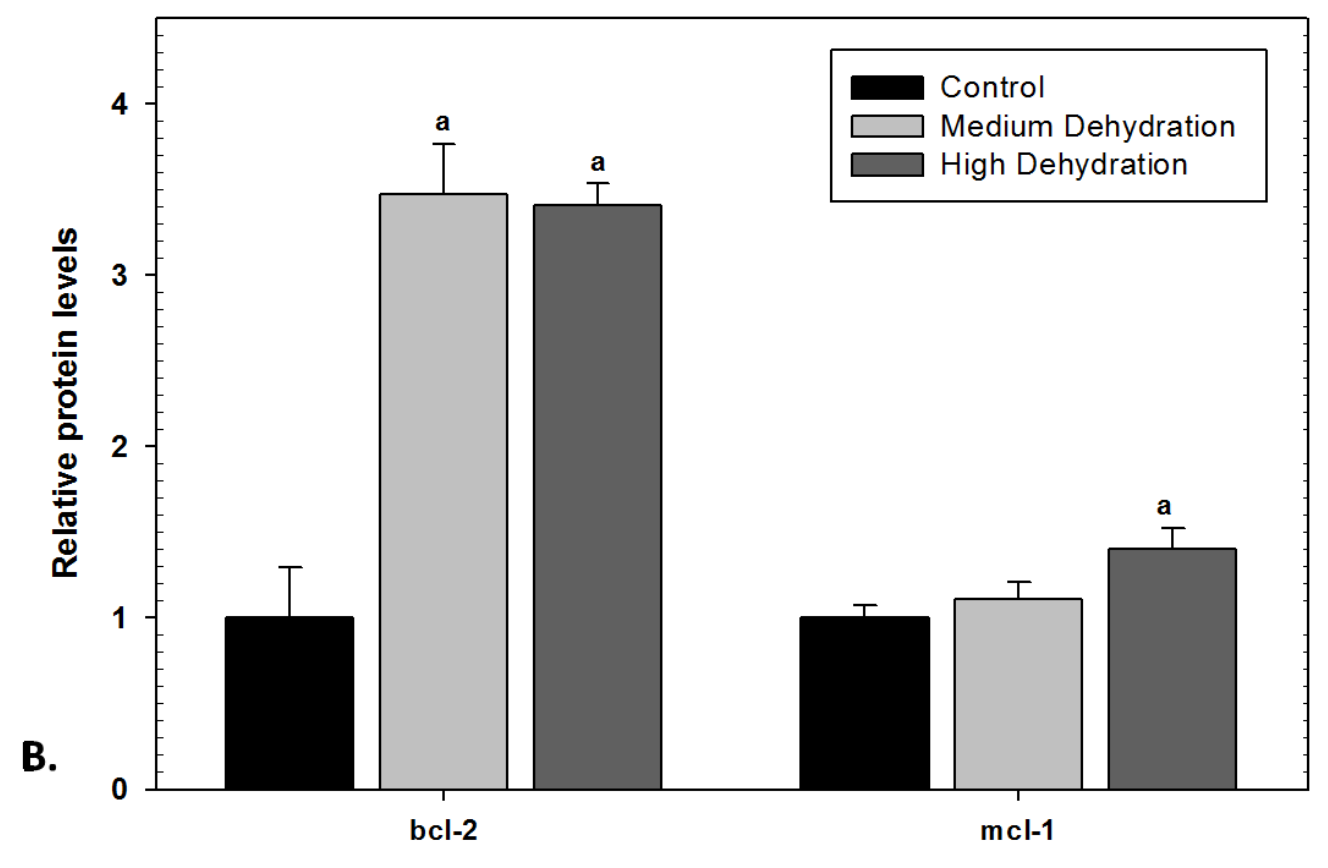

Control

Medium Dehydration

High Dehydration
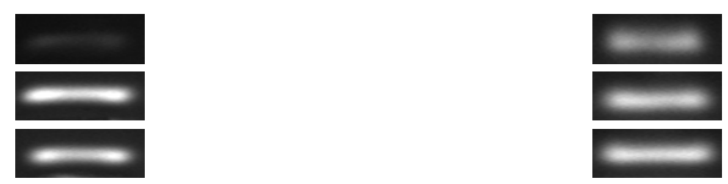

Figure 4.11: (A) Relative changes in mRNA transcript levels of STAT5 downstream targets; bcl-2 and mcl-1 in skeletal muscle under medium and high dehydration of $X$. laevis. Data are means \pm SEM for $\mathrm{n}=4$ independent trials. "a" - Significantly different from the control values $(P<0.05)$. (B) Representative PCR product bands visualized on a $1 \%$ agarose gel. 
A.

B.

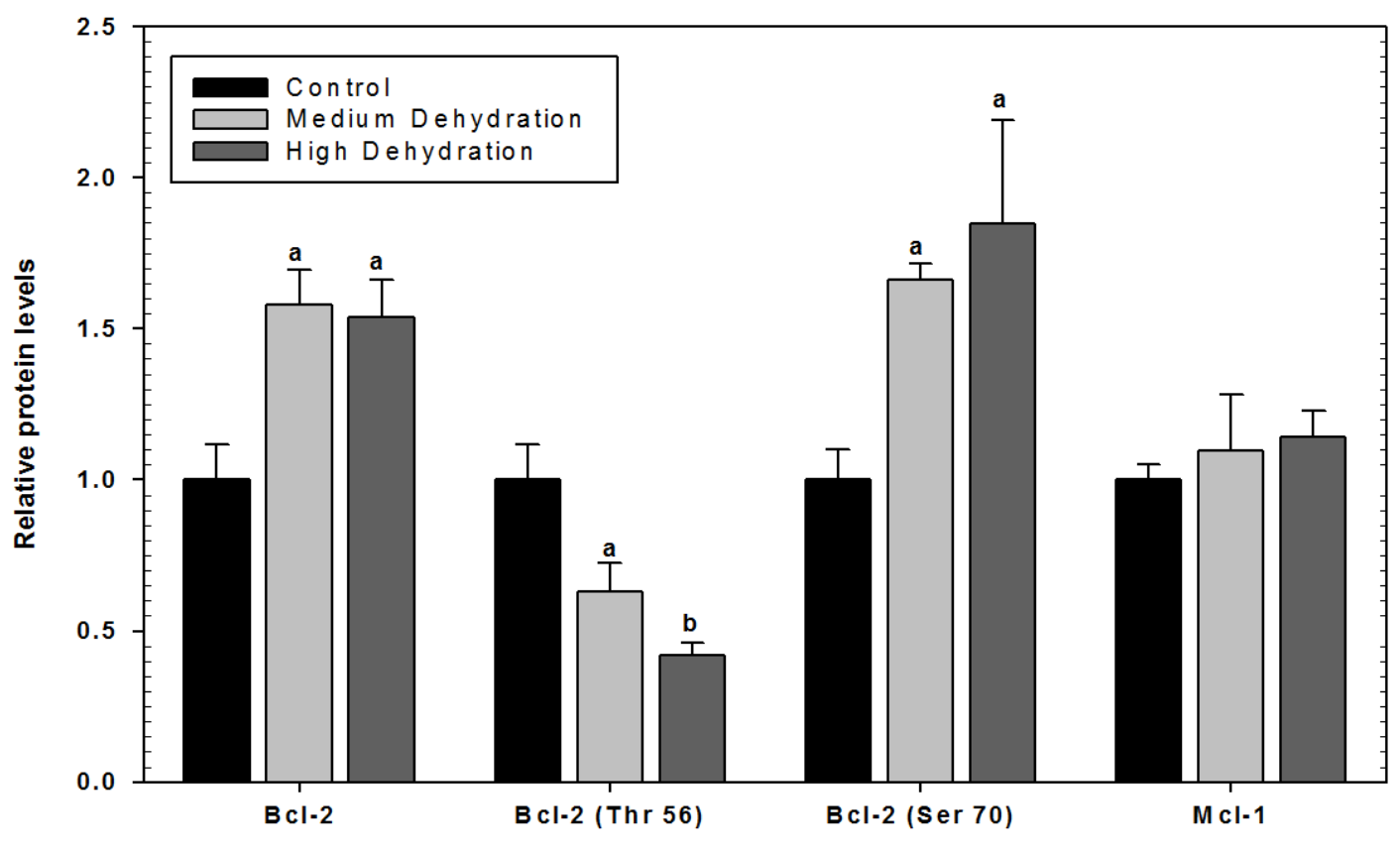

\section{Control}

Medium Dehydration

High Dehydration
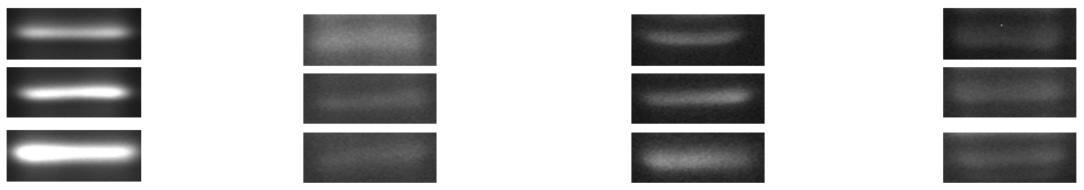

Figure 4.12: (A) Relative changes in protein levels of STAT5 downstream targets; total bcl-2 in addition to bcl-2 (Thr 56) and bcl-2 (Ser 70) in X. laevis skeletal muscle from medium and high dehydration conditions. Data are means \pm SEM for $\mathrm{n}=4$ independent trials. "a" - Significantly different from the control values $(P<0.05)$. Data are means \pm SEM for $\mathrm{n}=4$ independent trials. (B) Western blots showing representative bands. 


\section{References}

Alvarez, J.V. and D.A. Frank. 2004. Genome-wide analysis of STAT target genes: elucidating the mechanism of STAT-mediated oncogenesis. Cancer Biol Ther. 3(11): 1045-50.

Bhinge, A.A., Kim, J., Euskirchen, G.M., Snyder, M., and V.R. Iyer. 2007. Mapping the chromosomal targets of STAT1 by Sequence Tag Analysis of Genomic Enrichment (STAGE). Genome. Res. 17(6): 910-916.

Bromberg, J.F. 2001. Activation of STAT proteins and growth control. Bioessays. 23(2): 161-169.

De Chiara, G., Marcocci, M.E., Torcia, M., Lucibello, M., Rosini, P., Bonini, P., Higashimoto, Y., Damonte, G, Armirotti, A., Amodei, S., Palamara, A.T., Russo, T., Garaci, E., and F. Cozzolino. 2006. Bcl-2 Phosphorylation by p38 MAPK: identification of target sites and biologic consequences. J Biol Chem. 281(30): 21353-61.

Dechow, T.N., Pedranzin, L., Leitch, A., Leslie, K., Gerald, W.L., Linkov, I. and J.F. Bromberg. 2004. Requirement of matrix metalloproteinase-9 for the transformation of human mammary epithelial cells by Stat3-C. PNAS. 101(29): 10602-10607.

Grimley, P.M., Dong, F. and H. Rui. 1999. Stat5a and Stat5b: fraternal twins of signal transduction and transcriptional activation. Cytokine \& Growth Factor Rev. 10: 131-157.

Gross, A., McDonnell, J.M and S.J. Korsmeyer. 1999. BCL-2 family members and the mitochondria in apoptosis. Genes Dev. 13: 1899-1911.

Haller, O. and G. Kochs. 2002. Interferon-induced mx proteins: dynamin-like GTPases with antiviral activity. Traffic. 3(10): 710-717.

Ihle, J.N. 2001. The stat family in cytokine signaling. Curr Opin Cell Biol. 13(2): 211117.

Imada, K. and W.J. Leonard. 2000. The Jak-STAT pathway. Mol. Immunol. 37: 1-11.

Kristiansen, H., Scherer, C.A., McVean, M., Iadonato, S.P., Vends, S., Thavachelvam, K., Steffensen, T.B., Horan, K.A., Kuri, T., Weber, F., Paludan, S.R. and R. Hartmann. 2010. Extracellular 2'-5' oligoadenylate synthetase stimulates RNase L-independent antiviral activity: a novel mechanism of virus-induced innate immunity. Virol. 84(22): 11898-11904.

Ito, T., Deng, X., Carr, B., and W.S. May. 1997. Bcl-2 phosphorylation required for antiapoptosis function. J Biol Chem. 272(18): 11671-3.

Kroemer, G. 1997. The proto-oncogene Bcl-2 and its role in regulating apoptosis. Nat. 
Med. 3 (6): 614-619.

Lanuti, P., Bertagnolo, V., Pierdomenico, L., Bascelli, A. Santavenere, E., Alinari, L., Capitani, S., Miscia, S. and M. Marchisio. 2009. Enhancement of TRAIL cytotoxicity by AG-490 in human ALL cells is characterized by downregulation of cIAP-1 and cIAP-2 through inhibition of Jak2/Stat3. Cell Research. 19:1079-1089.

Michels, J., Johnson, P.W.M. and G. Packham. 2005. Mcl-1. IJBCB. 37: 267-271.

Mohapatra, S. Chu, B., Wei, S., Djeu, J., Epling-Burnette, P.K., Loughran, T., Jove, R. and W.J. Pledger. 2003. Roscovitine inhibits STAT5 activity and induces apoptosis in the human leukemia virus type 1-transformed cell line MT-2. Cancer Res. 63(23): 85238530 .

Park, C., Li, S., Cha, E., and C. Schindler. 2000. Immune response in STAT2 knockout mice. Immunity. 13(6): 795-804.

Sadler, A.J. and B.R. Williams. 2007. Structure and function of the protein kinase R. Curr Top Microbiol Immunol. 316: 253-292.

Schindler, C., Levy, D.E. and T., Decker. 2007. JAK-STAT signaling: from interferons to cytokines. J. Biol. Chem. 282: 20059-20063.

Socolovsky, M., Fallon, A.E.J., Wang, S., Brugnara' C. and H. F. Lodish. 1999. Fetal anemia and apoptosis of red cell progenitors in Stat5a-/-5b-/- mice: a direct role for Stat5 in Bcl-xL induction. Cell. 98: 181-191

Stark, G.R., Kerr, I.M., Williams, B.R., Silverman, R.H., and D.R. Schreiber. 1998. How cells respond to interferons. Annu. Rev. Biochem. 67: 227-264.

Storey, K.B. 2010. Out cold: biochemical regulation of mammalian hibernation - a minireview. Gerontology. 56(2): 220-230.

$\mathrm{Yu}, \mathrm{H}$. and R. Jove. 2004. The STATs of cancer- new molecular targets come of age. Nat Rev Cancer. 4(2): 97-105.

Wang, H.G., Rapp, U.R. and J.C. Reed. 1996. Bcl-2 targets the protein kinase Raf-1 to mitochondria. Cell. 87(4): 629-638.

Wen, Z., Zhong, Z., and J.E. Darnell. 1995. Maximal activation of transcription by Stat1 and Stat 3 requires both tyrosine and serine phosphorylation. Cell. 82(2):241-50.

Wruster, A.L., Tanaka, T. and M.J. Grusby. 2000. The biology of STAT4 and STAT6. Oncogene. 19: 2577-2584. 


\section{Chapter 5:}

\section{Expression of pro-apoptotic and anti- apoptotic miRNA targets in $X$. laevis liver and skeletal muscle during dehydration}




\section{Introduction}

The first microRNA (miRNA) was discovered by Lee et al. (1993) during studies of the metabolic pathways involved in the developmental stages of Caenorhabditis elegans. The researchers described the existence of a small, 22 base pair, non-coding RNA which bound directly to the 3'-untranslated region (3'UTR) of lin-14, a gene found to play an important role in the differentiation of specific lineages of C. elegans. The regulator was named lin-4 and was thought to be the only one of its kind until the discovery of let-7, another central regulator of developmental timing in C. elegans (Reinhart et al., 2000). Over the following two decades researchers identified these small, non-coding, RNA inhibitors in many other species including plants and viruses (Hwang and Mendell, 2006).

Currently, more than 1000 miRNAs have been identified in humans and are responsible for the regulation of about $30 \%$ of the genes in the mammalian genome. As an important regulator of gene expression, disruption of normal miRNA function has been described as a key characteristic of several human diseases, primarily concerning tumor development and progression (Hwang and Mendell, 2006).

It has been recently proposed that miRNAs may play a role in the transition into hypometabolic states since they represent a well coordinated, broadly applicable and easily reversible mode of regulation. The involvement of miRNAs in metabolic rate depression (MRD) has been examined in a number of animal models of hypometabolism including (1) mammalian hibernation, such as the 13-lined ground squirrel (Ictidomys tridecemlineatus) and little brown bat (Myotis lucifugus), (2) anoxia tolerance, through the study of intertidal snails (Littorina littorea), and (3) freeze tolerance, such as the wood frogs (Rana sylvatica) and painted turtles (Chrysemys picta). A number of miRNAs 
with functions in stress-response, suppression of protein synthesis, cellular proliferation and apoptosis were found to be differentially regulated in the hypometabolic state suggesting that miRNAs play crucial regulatory roles in MRD (Biggar and Storey, 2011; Abramyan et al., 2013). This chapter examined a possible regulatory role of miRNAs in another model of MRD, Xenopus laevis, a frog that is able to withstand substantial dehydration and estivates for weeks or months in its native land.

MiRNAs are initially transcribed by RNA polymerase II (pol II) as long transcripts, several thousand base pairs in length, termed primary-miRNA (Pri-miRNA; Figure 5.1). These transcripts are subsequently processes by Drosha, an RNAse III enzyme, into a 60-80 nucleotide stem loop hairpin structure called the precursor-miRNA (pre-miRNA; Jovanovic and Hengartner, 2006). Pre-miRNAs are exported into the cytoplasm where they are further processed by another RNAse III enzyme, Dicer, into a 18-24 nucleotide structure and bound to a miRNA-induced silencing complex (RISC). The miRNA acts as a guide that directs the RISC complex to target mRNA that is then either cleaved or translationally silenced (Subramanian and Steer, 2010). The binding of the miRNA to its target depends on the complementarity of the 3'UTR to the "seed region", a seven nucleotide sequence at base 2 to 8 in the $5^{\prime}$ end of the miRNAs. The seed region is highly conserved among species and is crucial for predicting miRNA targets (Cai et al., 2009). 


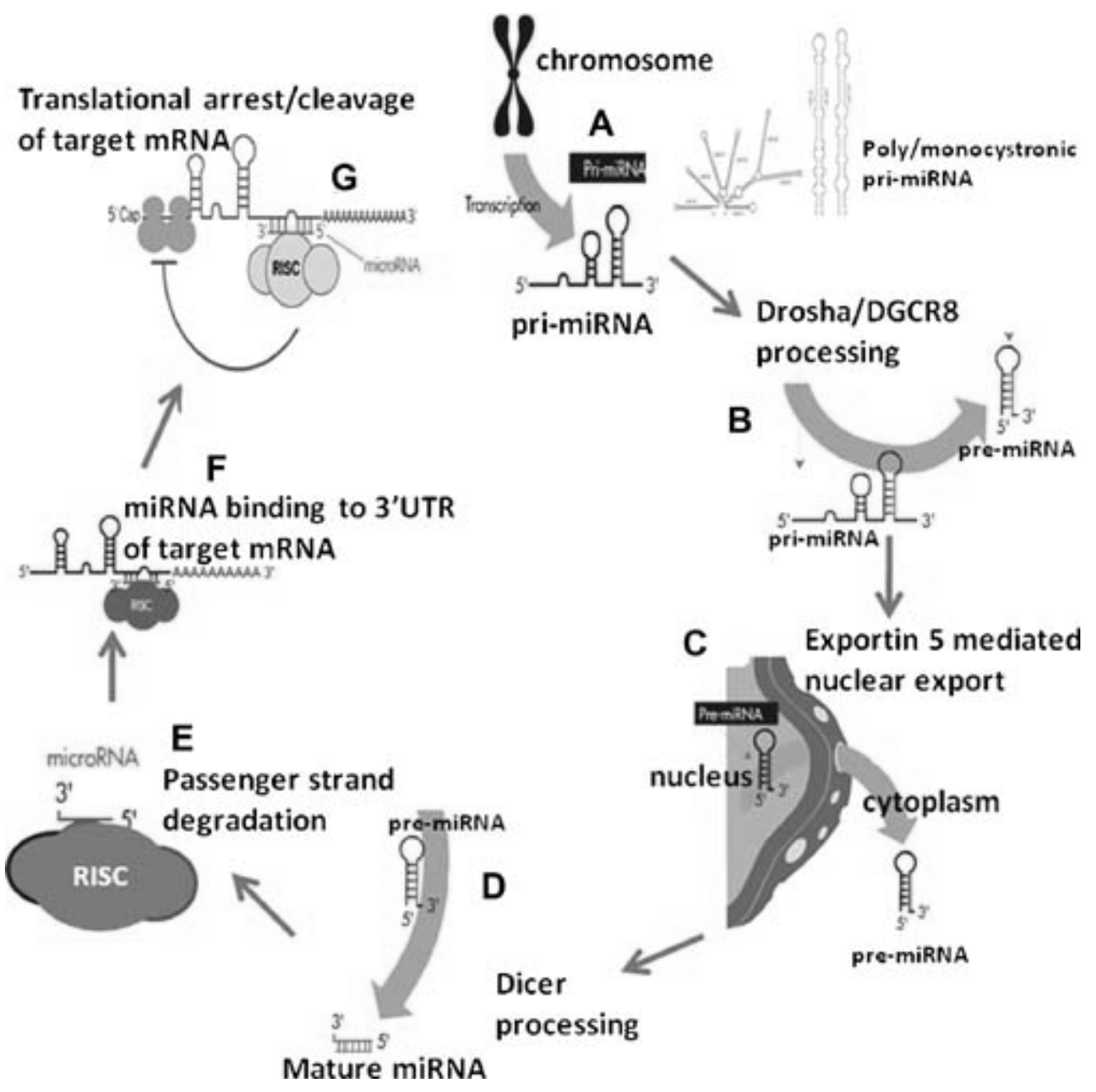

Figure 5.1: MiRNA biosynthesis. (A) RNA polymerase II transcribes primary-miRNAs followed by (B) processing by Drosha into a stem loop hairpin structure called premiRNA. (C)These transcripts are transported into the cytoplasm where they are processed by (D) Dicer into mature miRNAs and (E) bound to a miRNA-induced silencing complex (RISC). (F) miRNAs bind their target genes resulting in either (G) degradation or translational arrest (Subramanian and Steer, 2010). 
The fate of the targeted mRNA transcript depends on its base pairing to the miRNA. Perfect complementarity between the miRNA and its target results in cleaving of the mRNA by RISC, while imperfect base pairing results in translational repression of the transcript using cytoplasmic p-bodies or stress granules for storage. In response to an appropriate signal, the stored target mRNA is subsequently released from its inhibitory control and processed by the translational machinery into a functioning protein (Subramanian and Steer, 2010).

A single miRNA has the power to regulate multiple mRNA transcripts whereas each mRNA can be regulated by a number of different miRNAs. These interactions create a large network of interrelated pathways and mechanisms of regulation (Jevanovic and Hengartner, 2006). The complexity of this network makes it difficult to map specific miRNAs to specific targets, however in recent years, a large body of evidence has shown links between selected miRNAs and the regulation of apoptosis. Since the disruption of the normal growth and cell division processes is a major marker of cancer, research in this area has been focused on finding factors that influence the progression of the cell death program. To date more than 30 miRNAs have been implicated in the regulation of apoptosis through studies of human tumor growth and development (Subramanian and Steer, 2010).

The cell death program is largely carried out by caspases, a family of enzymes that catalyze the cleavage and degradation of several intracellular polypeptides important to the structural integrity of the cell and components of the DNA repair machinery (see Figure 1.1, Earnshaw et al, 1999). Briefly, the extrinsic pathway is initiated by a ligand binding to a death receptor (ex. FASR) which recruits adapter molecules to cleave 
activate caspase- 8 . The "initiator" caspase- 8 cleaves caspase $-3,-6$ and -7 to their active forms and unleashes their apoptotic potential (Dupont- Versteegden, 2005). The intrinsic pathway is activated by an internal stimuli which causes the mitochondria to release cytochrome $\mathrm{c}$ which joins the apoptotic protease activating factor-1 (Apaf-1), procaspase9, and dATP to form an apoptosome. This complex cleaves procascapase-3 to its active form thereby initiating the degradation of vital proteins and launches the apoptotic cascade (Zimmermann and Green, 2001).

Members of the Bcl-2 family serve as key players in the cell death pathway as they include pro-apoptotic members (Bax, BAD, BIM etc), that function to allow the release of damaging proteins from the mitochondria, and anti-apoptotic members (Bcl-2, Bcl-xL, Bcl-w, etc) that work to oppose this action (Adams and Cory, 1998).

Mechanisms that encourage cell survival and inhibit the apoptosis response are especially important during $X$. laevis estivation as this animal can spend months being immobile in its burrow. This kind of inactivity could result in severe muscle wasting in non-adapted animals as well as atrophy of liver (and intestinal) cells due to the unavailability of food (Hudson and Franklin, 2002; Cramp et al., 2005). MiRNAs with functions in the programmed cell death response may represent a vital layer of regulatory control that ensures that muscle and liver cells remain intact during dormancy to allow Xenopus frogs to resume their normal activity. The present chapter examines the responses to dehydration by three pro-apoptotic and four anti-apoptotic miRNAs in liver and muscle of $X$. laevis.

Pro-apoptotic miRNAs are those associated with genes whose protein products promote the cell death program; these miRNAs are generally down regulated in cancer 
cells (Subramanian and Steer, 2010). For example, mir-15a and mir-16 have been identified as pro-apoptotic miRNAs because they act as negative regulators of the antiapoptotic protein Bcl-2. These miRNAs have been found to be either deleted or down regulated in a large number of human cancers including chronic lymphocytic leukemia (CLL), pituitary adenomas, prostate carcinoma, gastric cancer, etc. (Cimmino et al., 2005; Ageilan et al., 2010; Xia et al., 2008). Evidence shows that the expression of both miRNAs are inversely correlated with the expression of the Bcl-2 protein, suggesting that during tumor development, a decrease in mir-15a and mir-16 results in the overexpression of Bcl-2 promoting cell survival. Similarly, studies of stellate cells (HSC) development in rat liver found that mir-15a and mir-16 were down regulated during development, whereas Bcl-2 protein expression was significantly increased (Guo et al., 2009). Oppositely, the administration of exogenous mir-15a and mir-16 transcripts resulted in significantly reduced Bcl-2 levels and increased the expression of caspase -3 , 8, and 9, thereby initiating apoptosis (Guo et al., 2009). Cimmino and colleagues (2005) found reduced levels of mir-15a and mir-16 expression in patients with CLL, while levels of bcl-2 were significantly elevated. The same study determined that both mir-15a and mir-16 regulate the expression of $b c l-2$ by directly binding to its 3'UTR sequence.

The third pro-apoptotic miRNA examined in this chapter is mir-101 that directly targets Mcl-1, another member of the Bcl-2 family that promotes cell survival ( $\mathrm{Su}$ et al., 2009). Mcl-1 functions to inhibit pro-apoptotic members of the family (Bim and Bak) that work to release cytochrome $\mathrm{c}$ and other damaging proteins from the mitochondria during the onset of the cell death program (Michaels et al., 2005). Mir-101 expression has been shown to be downregulated in several cancers such as hepatocellular carcinoma 
(HCC), lung cancer, and breast cancer and so it is considered to be a biomarker of tumor progression (Su et al., 2009; Yanaihara et al., 2006; Iorio et al., 2005).

The expression levels of the majority of known miRNAs are decreased in cancer cells, whereas a hand-full of miRNAs are typically consistently elevated. The latter miRNAs are being studied for their anti-apoptotic functions as they are thought to possess crucial roles in the uncontrolled growth and division of cancer cells (Hammond, 2006). A review by Subramanian and Steer (2010) discusses several miRNAs that have been well established via several lines of evidence to be anti-apoptotic. The relative abundance of four of these anti-apoptotic microRNAs (Subramanian and Steer, 2010) are analyzed in this chapter with respect to their possible involvement in apoptosis regulation in Xenopus under dehydration stress. These are mir-19b, mir-21, mir-92a, and mir-155.

One of the best-characterized oncogenic miRNA clusters is mir-17-92, also designated as oncomir-1 (Olive et al., 2010). The pre-miRNA derived from this gene is spliced into six mature miRNAs: miR-17, miR-18a, miR-19a, miR-20a, miR-19b-1, and miR-92-1. Initial evidence for the involvement of mir-17-92 in cancer came from studies in mice with B-cell lymphoma in which c-myc induced apoptosis was reduced by the introduction of mir-17-92 (Olive et al., 2010). By overexpressing mir-17-92, cellular death in the model was reduced and B-cell lymphoma development was accelerated. Similar experiments have been performed in a number of animal models, human cancer, and cell culture systems. Members of the mir-17-92 cluster have different functions including proliferation, apoptosis and angiogenesis (Olive et al., 2010). This chapter focuses on the expression of mir-19b and mir-92a since their function has been discovered to primarily involve preventing the onset of apoptosis. 
Mir-19b has been found to be a key oncogenic component of the mir-17-92 cluster as it specifically targets the gene encoding the PTEN (phosphatase and tensin) protein phosphatase, known as a tumor suppressor. This apoptotic protein is an inhibitor of the PI3K (phosphatidylinositol 3-kinase)-Akt-mTOR (mammalian target of rapamycin) pathway that is involved in cell survival and proliferation. Mir-19b targets the pten transcript, preventing it from being translated and allowing the PI3K-Akt-mTOR signaling pathway to proceed in its absence (Olive et al., 2009).

Recent studies found that mir-92a expression is elevated in many cancers, primarily functioning as an inhibitor of BIM, an apoptotic member of the Bcl-2 family of proteins (Croce, 2009). BIM interacts with the anti-apoptotic members of the Bcl-2 family such as Bcl-2 itself and Bcl-xL, opposing their function (O'Conner et al., 1998).

There are numerous studies that address the role of mir- 21 in the regulation of apoptosis and tumor growth, as it was found to be overexpressed in breast cancer, colorectal cancer, lung cancer and gastric cancer, among many others (Si et al., 2007; Asangani et al., 2008; Seike et al., 2009; Zhang et al., 2008). One of the main mechanisms through which mir-21 carries out its anti-apoptotic role is by targeting the gene RECK (reversion inducing cysteine-rich protein with kazal), a known tumor suppressing gene. This protein inhibits the action of metalloproteinases (MMPs) which play a critical role in the progression of tumor growth and metastasis. Mir-21 also regulates the expression PTEN, contributing to preventing cells from proliferating (Zhang et al., 2008). Additionally, mir-21 also targets the mRNA transcripts of key proteins including (1) PDCD4 (Programmed cell death protein 4) that is responsible for the inhibition of the activator protein-1 (AP-1) that enhances proliferation and differentiation, 
and (2) caspase-3, an executor of the cell death program (Krichevsky and Gabriely, 2009; Asangani et al., 2008). Other recognized targets of mir-21 include TPM1 (Tropomyosin alpha-1 chain), which is involved in muscle contraction, NFIB (nuclear factor I/B), which acts as a transcriptional repressor, and STAT3, a transcription factor whose gene targets include those with anti-apoptotic function (Krichevsky and Gabriely, 2009).

The last microRNA examined in this chapter is mir-155, which is overexpressed in colorectal cancer, as well as endometrial, ovarian, gastric, and urothelial tumors (Valeri, et al. 2010). It has been shown to bind and inhibit the mRNA encoding a proapoptotic target of the tumor suppressing transcription factor $\mathrm{p} 53$, tumor protein 53induced nuclear protein 1 (TP53INP; Gironella et al., 2007).

\section{Results}

\section{Analysis of pro-apoptotic miRNA levels in liver}

Three main miRNAs are generally considered to have pro-apoptotic functions; mir-15a, mir-16 and mir-101 (Figure 5.2). The relative transcript levels of these miRNAs in Xenopus liver were analyzed using stem-loop RT-PCR. Relative transcript levels of both mir-15a and mir101 transcripts significantly increased in the high dehydration condition, rising by $1.96 \pm 0.19$ fold and $1.28 \pm 0.04$ fold, respectively compared to controls $(P<0.05)$. No changes in relative levels of mir-16 were found between conditions. 


\section{Analysis of anti-apoptotic miRNA levels in liver}

Relative levels of the anti-apoptotic miRNAs, mir-19b, mir-21, mir-92a, and mir155, were also analyzed in Xenopus liver tissue (Figure 5.3). No significant changes in the relative transcript levels of mir-21 or mir-92a were found between the two dehydration conditions and controls. Relative transcript levels of mir-19b significantly increased in high dehydration, rising by $1.9 \pm 0.3$ fold compared to controls $(P<0.05)$. Significant changes were also found for mir-155, where relative levels rose by $2.61 \pm$ 0.09 fold under medium dehydration compared to controls. Transcript abundance subsequently decreased during HD to a level that was significantly lower but significantly higher than control values, rising by $1.4 \pm 0.1$ fold compared to controls $(P<0.05)$.

\section{Analysis of pro-apoptotic miRNA levels in skeletal muscle}

Relative transcript levels of mir-15a, mir-16 and mir- 101 were analyzed using stem loop RT-PCR in the skeletal muscle of Xenopus frogs (Figure 5.4). Mir-15a levels decreased significantly under medium dehydration, levels falling to $66 \pm 5 \%$ of controls $(P<0.05)$. Mir-16 levels decreased under high dehydrated conditions, falling to $67 \pm 6 \%$, of control values $(P<0.05)$. Relative levels of mir-101 were significantly increased in the high dehydration condition, rising by $1.65 \pm 0.07$ fold in the high dehydration condition compared to controls $(P<0.05)$.

\section{Analysis of anti-apoptotic miRNA levels in skeletal muscle}

Relative levels of the four anti-apoptotic miRNAs were also analyzed in Xenopus muscle (Figure 5.5). Only levels of mir-19b remained constant across all conditions. Relative levels of mir-21 significantly increased under the high dehydration condition, 
rising by $1.39 \pm 0.09$ fold compared to controls $(P<0.05)$. Relative levels of mir-92a decreased significantly in the medium dehydration condition, falling to $57 \pm 7 \%$ of controls, but subsequently rose during high dehydration to $1.83 \pm 0.03$ compared to controls and medium dehydration animals $(P<0.05)$. Finally, relative levels of mir-155 decreased significantly in medium dehydration, falling to $59 \pm 0.08 \%$ of controls $(P<0.05)$.

\section{Binding analysis}

A bioinformatics analysis was performed using the Targetscan algorithm to search for the presence within the 3' UTR of target genes of the binding sequence for each of the Xenopus miRs that were assessed. The $X$. laevis genome sequences available from NCBI proved to be too fragmented to correctly identify the 3'UTR in the Xenopus-specific target genes and so binding of $X$. laevis miRNAs was predicted based on the conserved sequences found in the 3'UTR of six mammal and one bird species: human (Homo sapiens), common chimpanzee (Pan troglodytes), nine-banded armadillo (Dasypus novemcinctus), lesser hedgehog tenrec (Echinops telfair), house mouse (Mus musculus), red junglefowl (Gallus gallus), and a brown rat (Rattus norvegicus). Figures 5.6 and 5.7 show results for the three pro-apoptotic miRNAs. Fig. 5.6A shows the presence of a binding sequence in the 3' UTR of $b c l-2$ that is fully conserved in the gene from all six mammal and bird sequences and that is fully complementary with the 5' region of $X$. laevis miR-15a and miR-16-1. Similarly, the binding sequence in the 3' UTR of mammalian $M c l-1$ sequences was conserved and complementary to the 5' sequence of miR-101 (Fig.5.7). Comparable results were found for the four anti-apoptotic miRNAs 
when comparing mir-19b binding to $p t e n$, miR-21 binding to $p d c d 4$, miR-92a binding to bim, and miR-155 binding to tp53inp (Fig. 5.8-5.11, respectively).

The RNAhybrid algorithm was used to statistically determine the thermodynamic stability of the miRNA:target complex. A cutoff binding energy of $-18 \mathrm{~kJ}$, was used to confirm the binding likelihood of the miRNAs to the conserved binding site (Singh and Nagaraju, 2008; Bartel, 2009). The binding energies of mir-15 to $b c l-2$, mir-16 to $b c l-2$ and mir-101 to $m c l-1$ were $-23.1,-25.5$, and -20.1 and whereas comparable binding energies of mir-19b to $p t e n$, mir-21 to $p d c d 4$, mir-92a to bim, mir-155 to tp53inp were $-27.2,-17.8,-18.3$, and $-18.4 \mathrm{kcal} / \mathrm{mol}$, respectively.

\section{Discussion}

The descent into a hypometabolic state is a fascinating animal adaptation to environmental stress (Storey and Storey, 2011). Hypometabolism is characterized by a significant decrease in overall metabolic rate (20-30\% percent of normal resting rate), a reorganization of the cellular landscape so as to conserve energy, and enhanced expression of a small number of pathways vital for survival (Storey and Storey, 2010). This incredible feat requires rapid, global, and reversible controls to quickly reprioritize cellular pathways and prepare the organism for entry into dormancy. One such mode of regulatory control occurs at the post-translational level by the reversible phosphorylation of multiple proteins including metabolic enzymes, key transcription factors and transmembrane transporters with important functions for survival (Storey and Storey, 2011).

Recent studies propose another mode of regulation during metabolic rate depression occurring at the post-transcriptional level, through the action of small non- 
coding RNAs called microRNAs (Biggar and Storey, 2011). These transcripts are able to bind to the 3'UTR of their target genes and direct the RNA-induced silencing complex (RISC) to induce mRNA degradation (Subramanian and Steer, 2010). Another option of miRNA regulation is translational repression occurring when the miRNA sequence is only partially complimentary to its target. The target mRNA is then stored in a p-body until a signal stimulates the miRNA to release it from its inhibitory control and allow translation to proceed (Subramanian and Steer, 2010). Regulation by miRNAs seems to be a good candidate for a global regulatory control during descent into and arousal from dormant states. This mode of regulation is broadly applicable, highly coordinated, easily reversible, and extremely energy efficient by simply storing mRNA until a time when its translation can be reinitiated (Biggar and Storey, 2011).

This chapter examined the expression of seven miRNAs, three with pro-apoptotic functions and four with anti-apoptotic functions, in skeletal muscle and liver tissues of control and dehydrated X. laevis. The miRNAs chosen for study were those that have established vital functions in the regulation of the cellular cell death pathway. In a recent review, Subramanian and Steer (2010) identified over 30 miRNAs that have been implicated in the regulation of apoptosis via several lines of evidence. These include proapoptotic miRNAs that are responsible for the inhibition of genes whose protein products act to fight the cellular death program and have important functions in cell survival. Three such miRNAs were analyzed in this study; mir-15a, mir-16 and mir-101.

Mir-15a and mir-16 have been identified as regulators of the progression of the cell cycle as well as cell survival. Their target genes include proteins that are essential to the proper progression of cell cycle such as cyclin D1, cyclin E, cdc25a, and E2F1 in 
addition to the anti-apoptotic protein bcl-2. Studies where exogenous amounts of these two miRNAs were introduced led to a significant decrease in the total number of cells as well as an increase in the critical initiators and executers of cellular death; caspase- 3, 8, and 9. These cells also displayed reduced levels of bcl-2 indicating that an increase in mir-15 and mir-16 is a key event in the controlled cell death pathway (Guo et al., 2009).

In this study, expression levels of mir-15a were significantly elevated in the liver of the highly dehydrated X. laevis compared to controls (Figure 5.2). In contrast, in skeletal muscle levels of mir-15a significantly decreased in medium dehydration and levels of mir-16 significantly decreased in high dehydration compared to controls (Figure 5.4). Studies of another model of metabolic rate depression, a wood frog that sustained freezing for 24 hours, found increased levels of mir-16 transcripts in the liver. The authors proposed that the elevated levels of mir-16 may serve to inhibit hepatocytes from proliferating under stress conditions in order to preserve energy for the arousal stage (Biggar et al., 2009). A similar explanation may be true for the hepatocytes of dehydrated Xenopus, where mir-15a may be upregulated to inhibit cell division in order to conserve energy reserves.

The prevention of skeletal muscle atrophy during the dormant period is extremely important as muscle loss can threaten Xenopus' reproductive success and the ability to escape its predators upon arousal (Hudson and Franklin, 2002). It is possible that mir-15a and mir-16 are differentially regulated in this tissue compared to liver because stronger regulatory controls are required to prevent muscle wasting and as the preservation of skeletal muscle is crucial for Xenopus survival, the limited available energy is allocated for this function. 
Similar findings were described in the anoxia tolerant red-eared slider turtle (Trachemys scripta elegans) where levels of both mir-15a and mir-16 increased in the liver but remained constant in the white skeletal muscle. The same study found that the expression of both microRNAs was inversely correlated with the expression levels of cyclin D1, a critical regulator of the G1 phase of the cell cycle and considered to be a main initiator of cell division (Biggar and Storey, 2012). Perhaps similar regulatory pathways are taking place in the liver and skeletal muscle tissues of dehydrated Xenopus.

The third pro-apoptotic miRNA examined in this study is mir-101 that is typically downregulated in human cancers due to its function in the cell death pathway. Its main targets include mcl-1, a member of the bcl-2 family, that works to inhibit the proapoptotic members of the family Bim and Bak (Su et al., 2009). By binding to the 3'UTR sequence of mcl-1, mir-101 silences its action and allows Bim and Bak to initiate the cell death response by releasing cytochrome $\mathrm{C}$ from the mitochondria (Michaels et al., 2005). Mir-101 transcripts were elevated under highly dehydrated conditions in both liver and skeletal muscle tissues (Figure 5.2 and 5.4). Again, the results for liver may indicate a mechanism of energy conservation where hepatocyte cells are prevented from dividing under the stress of dehydration. Further studies are required to assess the purpose of the upregulation of a pro-apoptotic miRNA during Xenopus dehydration in two of its most vital tissues.

Vital miRNA transcripts that are targeted by mir-21 include the well-known tumor suppressors RECK and PTEN, as well as PDCD4 (Programmed cell death protein 4, a gene is responsible for inhibition of proliferation), as well as caspase-3 (an executer of the cell death program). After the onset of a cell death stimulus, caspase-3 is cleaved to 
its active form and subsequently degrades vital polypeptides important to the structural integrity of the cell and components of the DNA repair machinery (Earnshaw et al., 1999). Mir-21 expression levels were elevated in the highly dehydrated condition in the skeletal muscle tissue while no changes were recorded in liver (Figure 5.3 and 5.5).

Studies with frozen wood frogs reported an increase in mir-21 levels in both liver and skeletal muscle while studies with hibernating 13-lined ground squirrels found elevated levels of mir-21 in kidney (Biggar et al., 2009; Morin et al., 2008). Taken together these findings suggest that activation of the mir-21 miRNA may be a conserved mechanism in the regulation of metabolic rate depression.

One of the best described oncogenic group of miRNAs is the mir17-92 cluster which consists of six mature miRNAs: miR-17, miR-18a, miR-19a, miR-20a, miR-19b-1, and miR-92-1. Together this family of miRNAs is responsible for the regulation of genes with functions in cell proliferation, cell survival as well as angiogenesis (Olive et al., 2010). This chapter analysed the relative expression patterns of two members of the family, mir$19 \mathrm{~b}$ and $-92 \mathrm{a}$. In the liver mir-19b transcript levels significantly increased in the high dehydration condition whereas mir-92a levels remained constant. In skeletal muscle relative levels of mir-19b remained the same across conditions while levels of mir-92a significantly decreased during medium dehydration but increased significantly in the high dehydration condition.

These data seem to indicate that there is a tissue dependent regulation of these two members of the mir-17-92 cluster. Mir-19b is a known regulator of the well known tumor suppressor, PTEN that works to suppress the cellular levels of PI3K which is a key player in promoting cell growth, proliferation, differentiation and survival. One of the main 
PTEN functions involves the activation of the Akt-mTOR pathway that is required for cell cycle progression and has been shown to inhibit programmed cell death, presumably by targeting eIF4E, a protein that promotes cell survival in several studies with cell lines and human cancer cells (Hay and Sonenberg, 2004).

$\mathrm{BIM}$ is an apoptotic member of the bcl-2 family that acts to release cytochrome $\mathrm{C}$ from the mitochondria thereby initiating the cell death sequence. Its action is opposed by mir-92a which directly binds to its 3'UTR sequence (Croce, 2009). Both mir-19b and mir-92a are members of the same cluster of proteins that function to prevent apoptosis and promote cell survival through two distinct cellular pathways. Further studies are required to explain why these two miRNAs are differentially regulated in the liver and muscle of dehydrated Xenopus. One explanation may be that the direct inhibition of the pro-apoptotic BIM is a faster method of preventing the onset of cellular death compared to the indirect activation of anti-apoptotic proteins through the AKT-mTOR pathway. It is possible that once the dehydration levels become critical, the skeletal muscle tissue requires immediate and efficient action to prevent muscular atrophy.

Suppression of muscle wasting is extremely important during the dormancy season of Xenopus. The frogs must retain their muscle mass and contractile power as this is vital for reproductive success and the ability to escape from predators upon arousal. Studies with the estivating green-striped burrowing frog (Cyclorana alboguttata) have provided evidence for extremely limited muscle atrophy occurring during estivation when compared to other models of muscle disuse (Hudson and Franklin, 2002).

Mir-155 expression levels were also found to be differentially regulated in the liver and skeletal muscle of Xenopus. In the liver mir-155 expression levels increased 
significantly under medium dehydration followed by a drop in the high dehydration condition to levels that were still significantly higher than control animals. In skeletal muscle levels of mir-155 dropped in the medium dehydration condition. Mir-155 is less understood than the other miRNAs examined in this study however it is considered a vital anti-apotptotic target as it was found to be overexpressed in many cancers where its main gene target is TP53INP, a pro-apoptotic gene under the regulation of the tumor suppressing transcription factor, p53 (Valeri, et al. 2010; Gironella et al., 2007).

A bioinformatics analysis was carried out to validate that the miRNAs discussed in this chapter are able to bind their target genes through Watson-Crick base pairing, thermodynamics, and conservation analysis (Figure 5.6- 5.11). The TargetScan program was used to identify gene targets with perfect complementary base pairing to the chosen miRNA sequences in their seed region, a seven nucleotide sequence at base 2 to 8 in the 5 ' end of the miRNAs (Cai et al., 2009). This program was also used to conduct a conservation analysis of the miRNAs from humans (Homo sapiens), chimpanzees (Pan troglodytes), armadillo (Dasypus novemcinctus), hedgehogs (Echinops telfair), mice (Mus musculus), rats (Rattus norvegicus) and chickens (Gallus gallus). The seed region of the three miRNAs was found to be conserved between all species examined.

The thermodynamics of the miRNA:target complex are critical to its stability. To determine the thermodynamic stability, the RNAhybrid algorithm was used to statistically determine the most energetically favorable hybridization sites. A cutoff binding energy of $-18 \mathrm{kcal} / \mathrm{mol}$, was used to confirm the binding likelihood of the miRNAs to the conserved binding site (Singh and Nagaraju, 2008; Bartel, 2009). The bioinformatics analysis of all seven miRNAs found binding energies within the accepted 
range, although mir-21 was a little under with a binding energy of $-17.8 \mathrm{kcal} / \mathrm{mol}$. Taken together with the binding and conservation analyses, the thermodynamic binding estimates suggest that the miRNAs discussed in this chapter are able to regulate the expression of their proposed target genes: mir-15/16 and $b c l-2$, mir-19b and pten, mir-21 and $p d c d 4$, mir-92a and bim, mir-101 and $m c l-1$, and mir-155 and tp53inp). Moreover, the binding analysis predicted imperfect binding between the miRNAs and their targets suggesting a mechanism for translational silencing and not target degradation. Further analysis of the miRNA regulation of their specific targets will be addressed in Chapter 6 .

Overall, this chapter analyzed the expression levels of seven miRNAs vital for the regulation of apoptosis during periods of stress. It was found that during Xenopus dehydration pro-apoptotic miRNAs generally decreased in the skeletal muscle tissue and increased in the liver tissue. The examined anti-apoptotic miRNAs were upregulated in a tissue dependent manner, some were found to be elevated in skeletal muscle while others were elevated in liver. Further studies are required to explain the tissue dependent activation of anti-apoptotic agents however this chapter provided novel insight into miRNA regulation during metabolic rate depression in Xenopus. 
A.

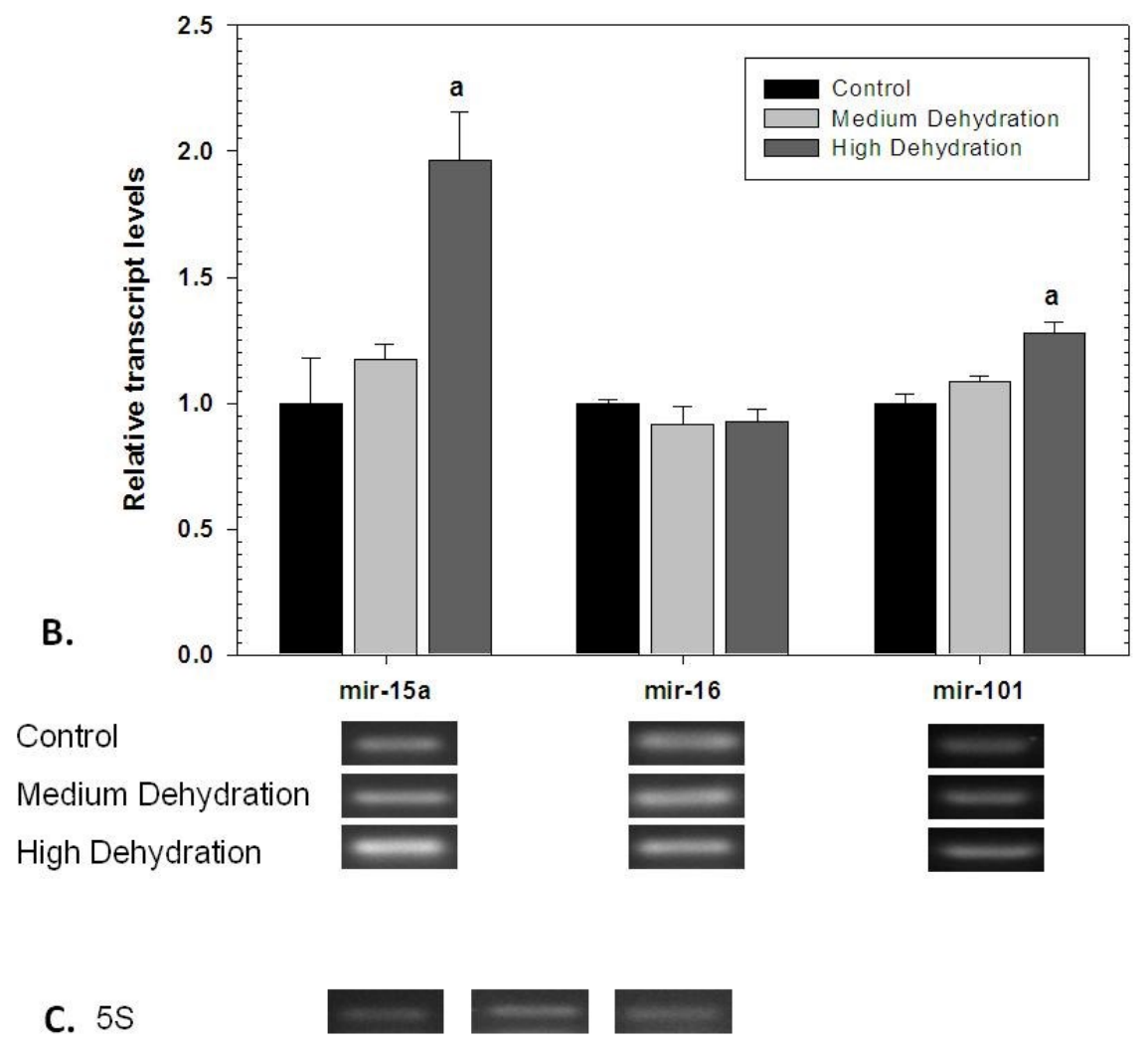

Figure 5.2: (A) Relative changes in the transcript levels of three pro-apoptotic miRNAs, mir-15a, mir-16 and mir-101, in liver in response to medium and high dehydration of $X$. laevis. Data are means \pm SEM for $\mathrm{n}=4$ independent trials. "a" - Significantly different from the control values $(P<0.05)$. (B) Representative PCR product bands visualized on a $1 \%$ agarose gel. (C) Representative $5 \mathrm{~S}$ bands used to standardize Mir bands. 
A.

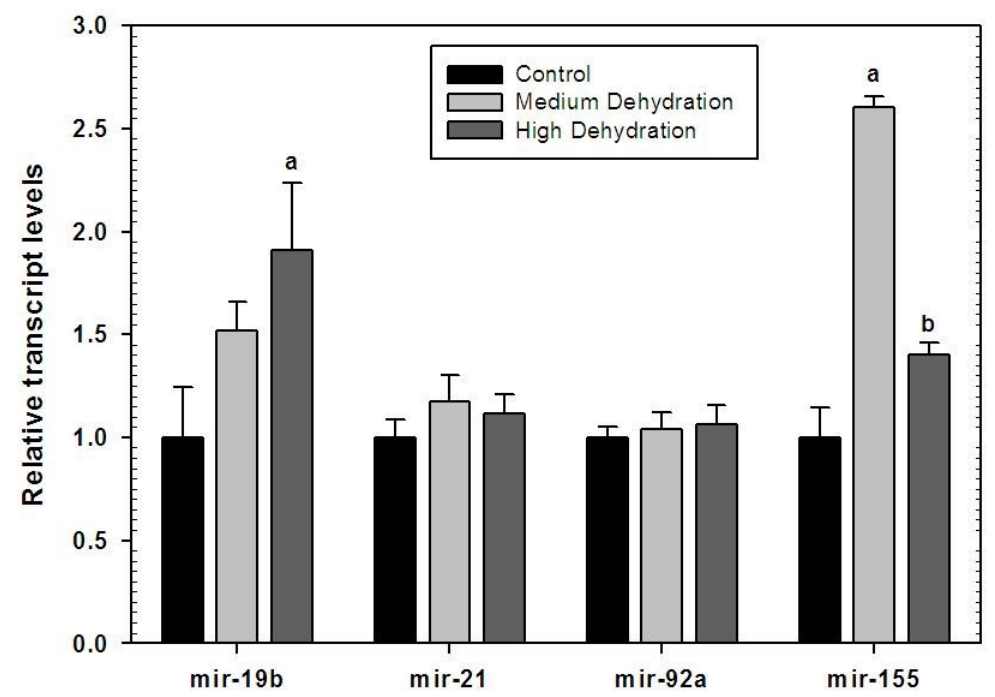

B.

Control

Medium Dehydration

High Dehydration
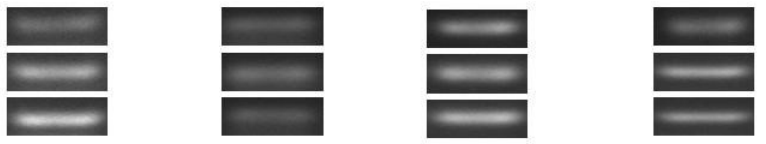

C. $5 S$

Figure 5.3: (A) Relative changes in the transcript levels of four anti-apoptotic miRNAs, mir-19b, mir-21, mir-92a, and mir-155, in liver in response to medium and high dehydration of $X$. laevis. Data are means \pm SEM for $n=4$ independent trials. "a" Significantly different from the control values, "b" - significantly different from control and medium dehydration $(P<0.05)$. (B) Representative PCR product bands visualized on a 1\% agarose gel. (C) Representative 5S bands used to standardize Mir bands. 
A.

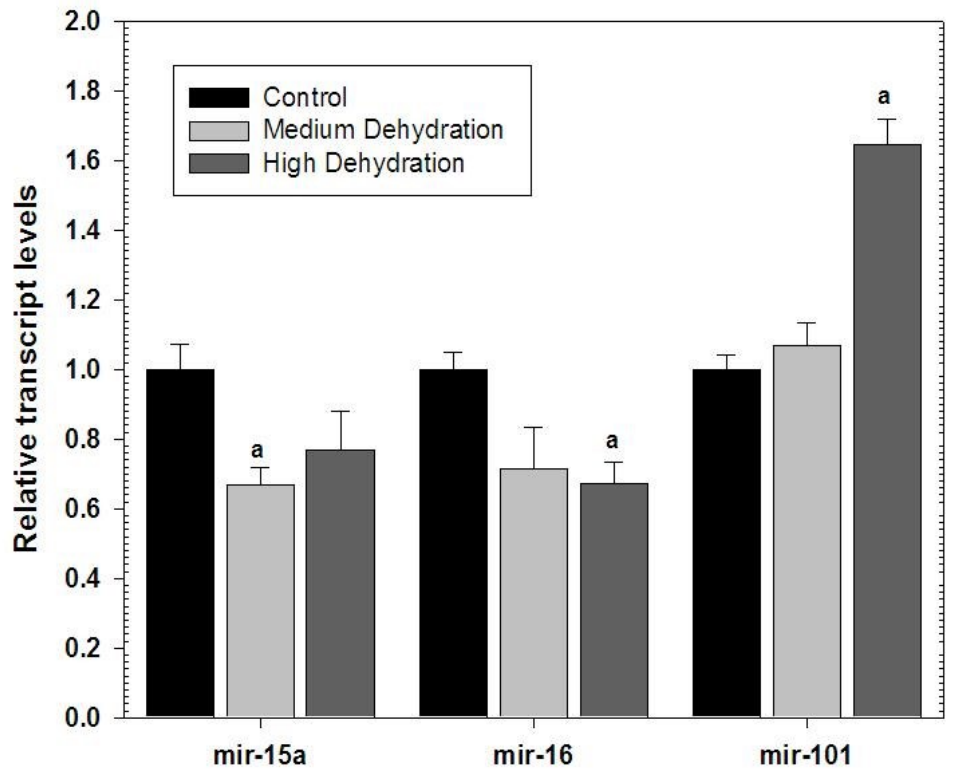

B.

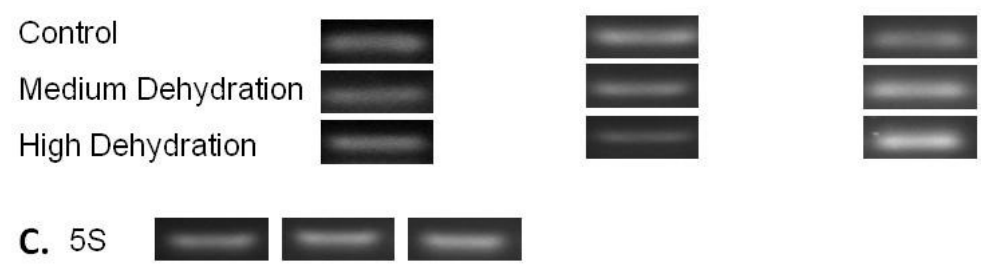

Figure 5.4: (A) Relative changes in the transcript levels of three pro-apoptotic miRNAs, mir-15a, mir-16 and mir-101, in skeletal muscle in response to medium and high dehydration of $X$. laevis. Data are means \pm SEM for $\mathrm{n}=4$ independent trials. "a" Significantly different from the control values $(P<0.05)$. (B) Representative PCR product bands visualized on a 1\% agarose gel. (C) Representative 5S bands used to standardize Mir bands. 
A.

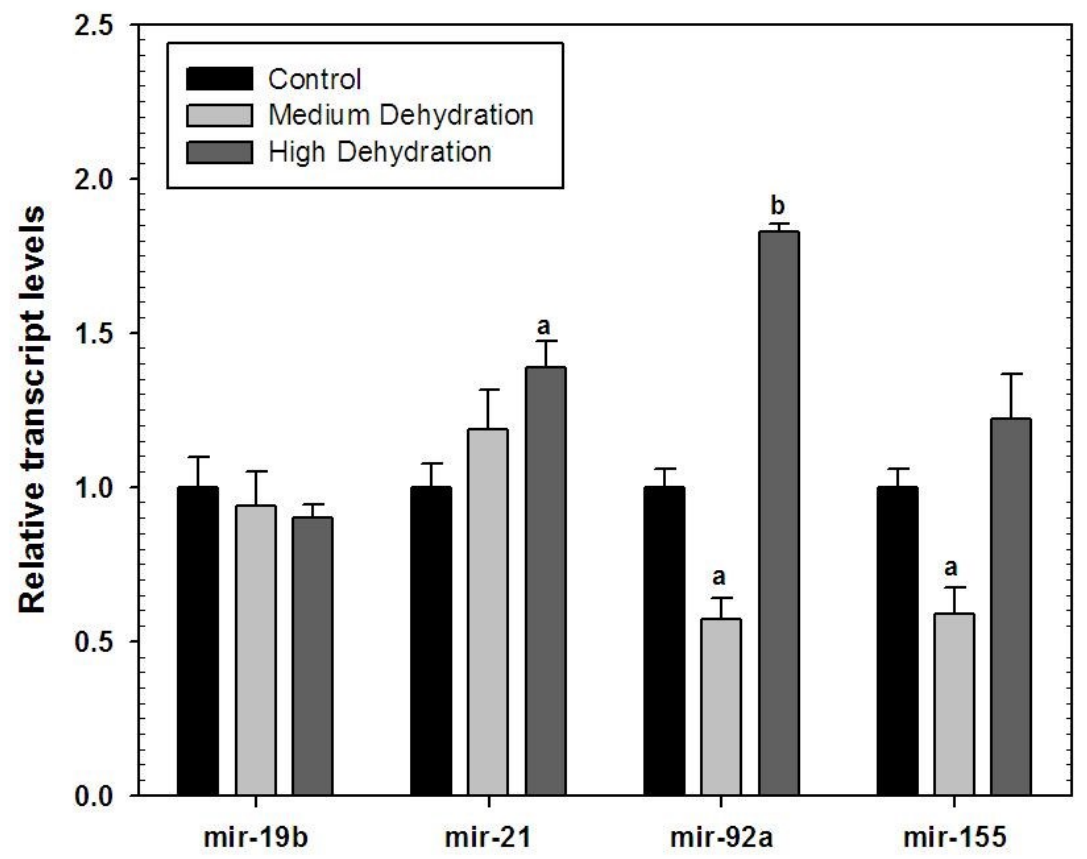

B.

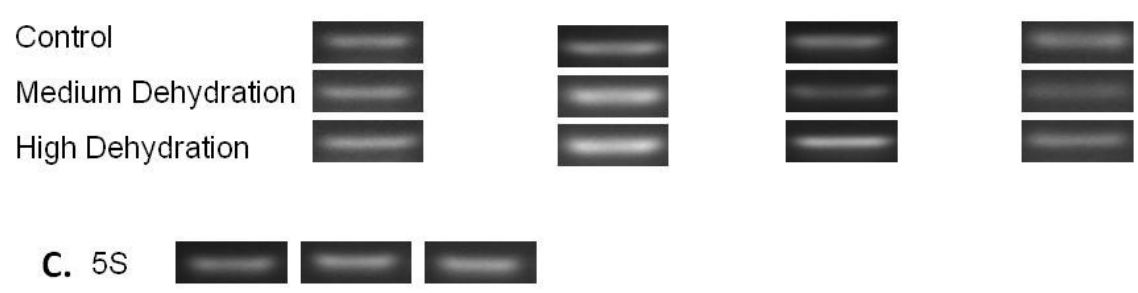

Figure 5.5: (A) Relative changes in the transcript levels of four anti-apoptotic miRNAs, mir-19b, mir-21, mir-92a, and mir-155, in skeletal muscle in response to medium and high dehydration of $X$. laevis. Data are means \pm SEM for $\mathrm{n}=4$ independent trials. "a" Significantly different from the control values $(P<0.05)$. (B) Representative PCR product bands visualized on a 1\% agarose gel. (C) Representative 5S bands used to standardize Mir bands. 
A

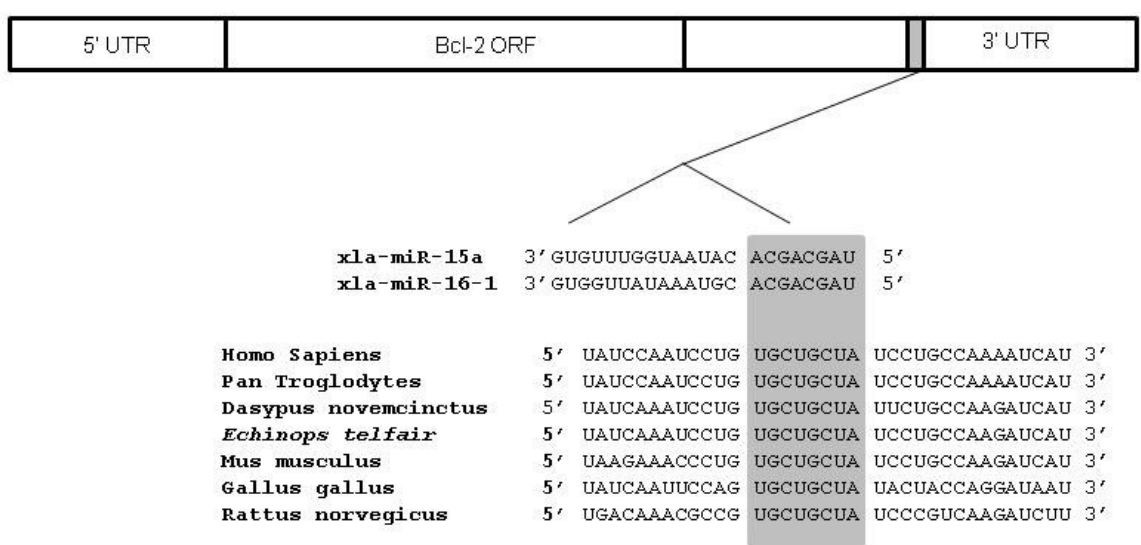

B

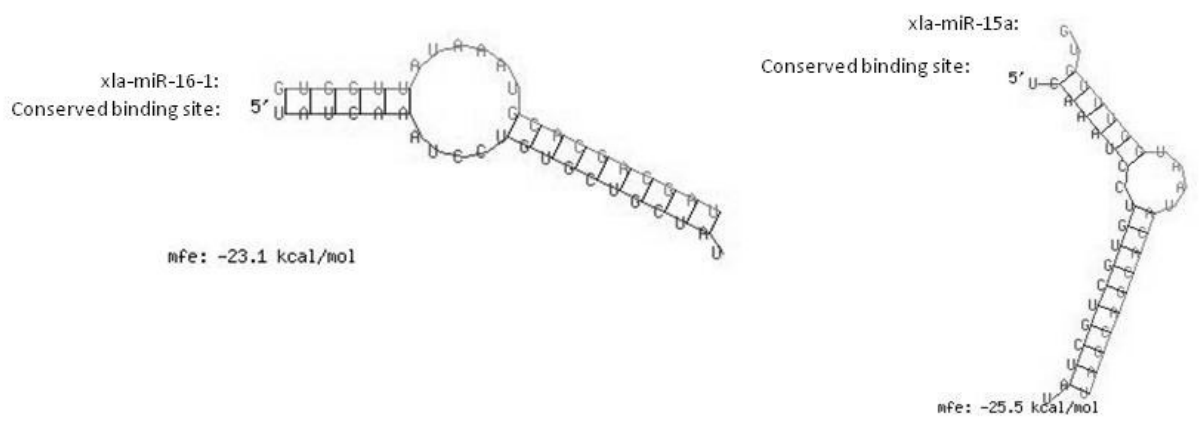

Figure 5.6: (A) Conservation analysis of the mir-15 and mir-16 binding sequence in the 3' UTR of $b c l-2$. (B) Predicted binding structures of Xenopus specific mir-15a and mir16 to the 3'UTR of $b c l-2$ with predicted binding energies (generated by RNAhybrid). 
A

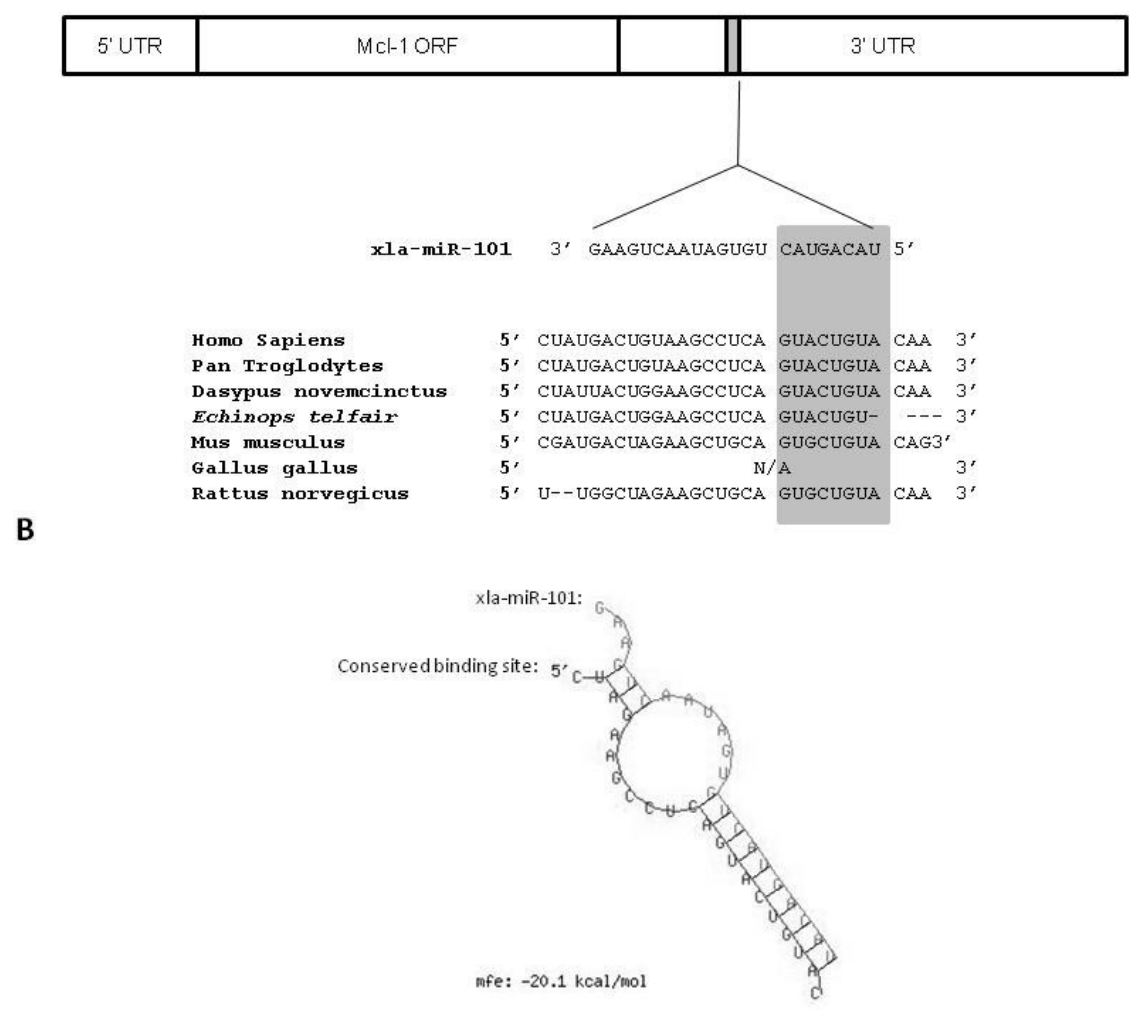

Figure 5.7: (A) Conservation analysis of the mir-101 binding sequence in the 3 ' UTR of $m c l-1$. (B) Predicted binding structures of Xenopus specific mir-101 to the 3'UTR of $m c l$ 1 with predicted binding energies (generated by RNAhybrid). 
A
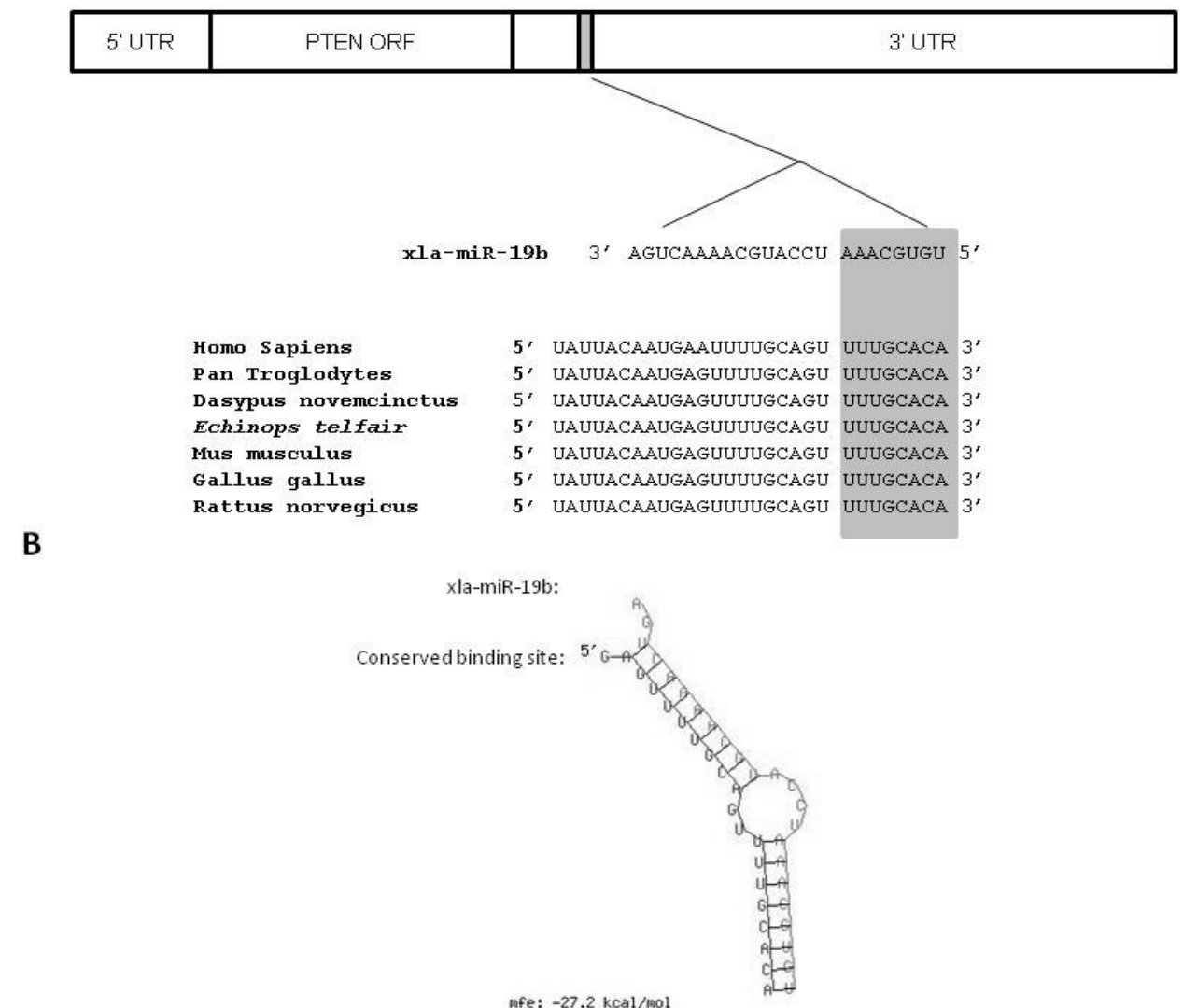

Figure 5.8: (A) Conservation analysis of the mir-19b binding sequence in the 3 ' UTR of pten. (B) Predicted binding structures of Xenopus specific mir-19b to the 3'UTR of pten with predicted binding energies (generated by RNAhybrid). 
A
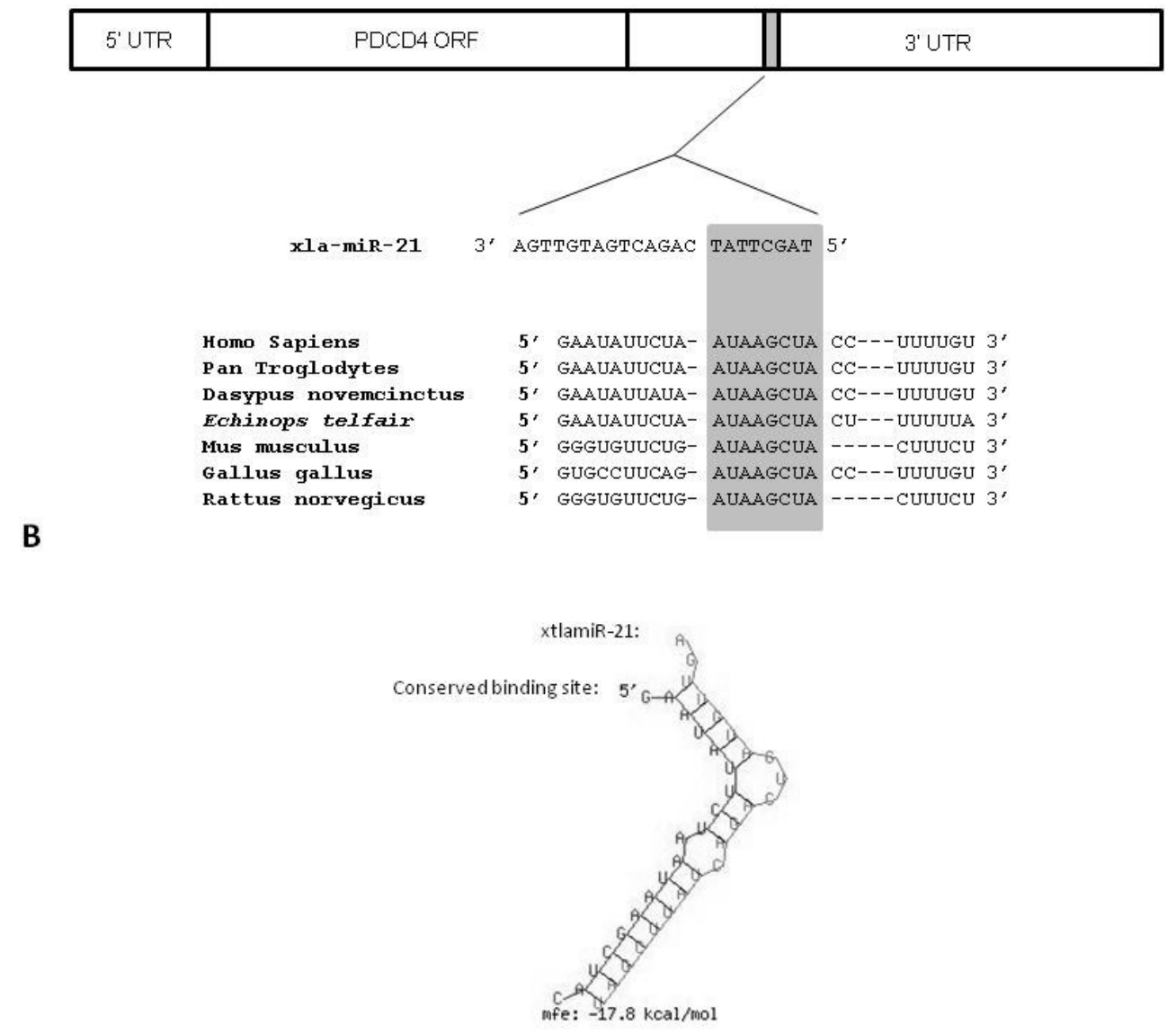

Figure 5.9: (A) Conservation analysis of the mir-21 binding sequence in the 3 ' UTR of pdcd4. (B) Predicted binding structures of Xenopus specific mir-21 to the 3'UTR of $p d c d 4$ with predicted binding energies (generated by RNAhybrid). 
A
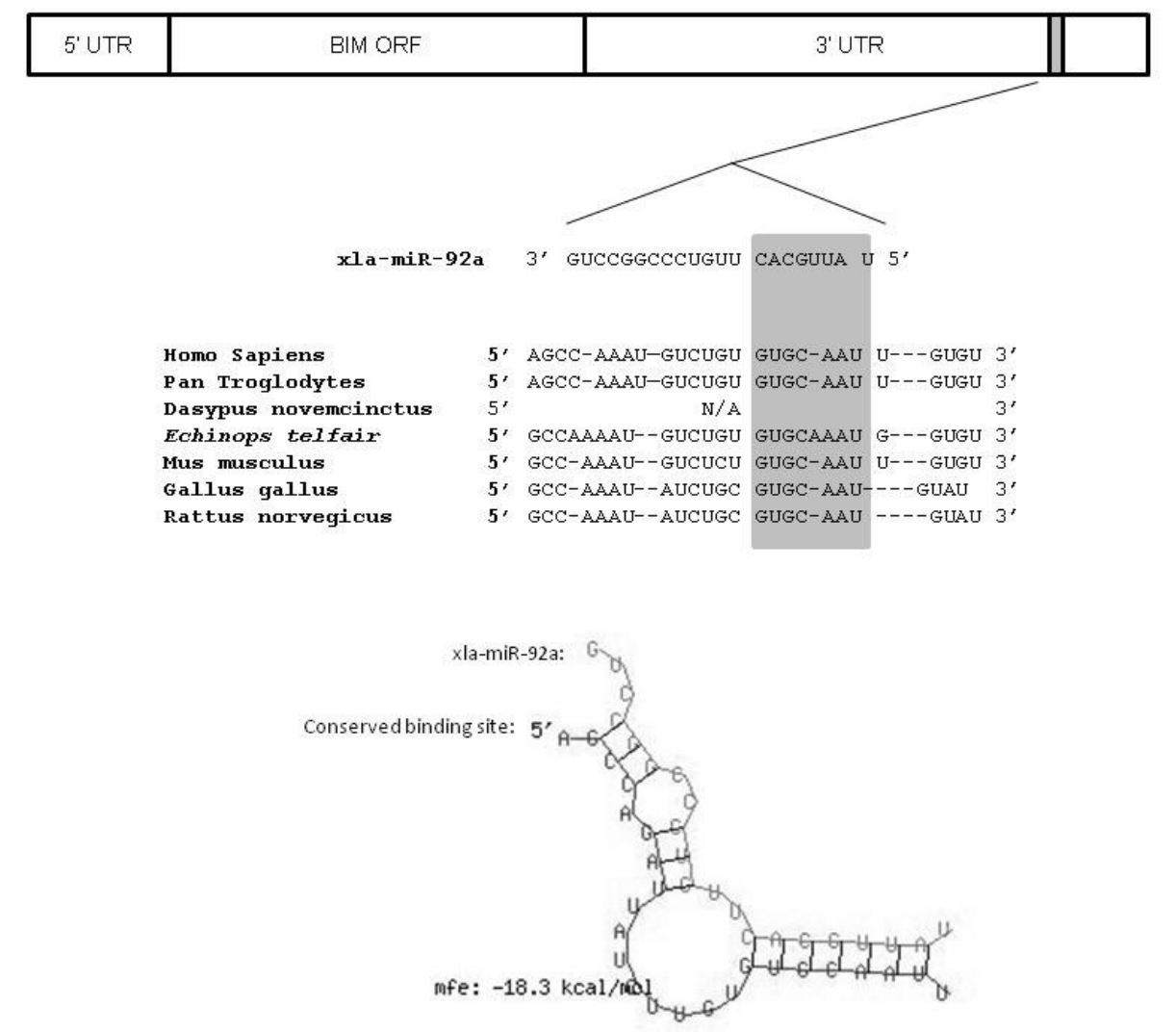

Figure 5.10: (A) Conservation analysis of the mir-92a binding sequence in the 3 ' UTR of bim. (B) Predicted binding structures of Xenopus specific mir-92a to the 3'UTR of bim with predicted binding energies (generated by RNAhybrid). 
A

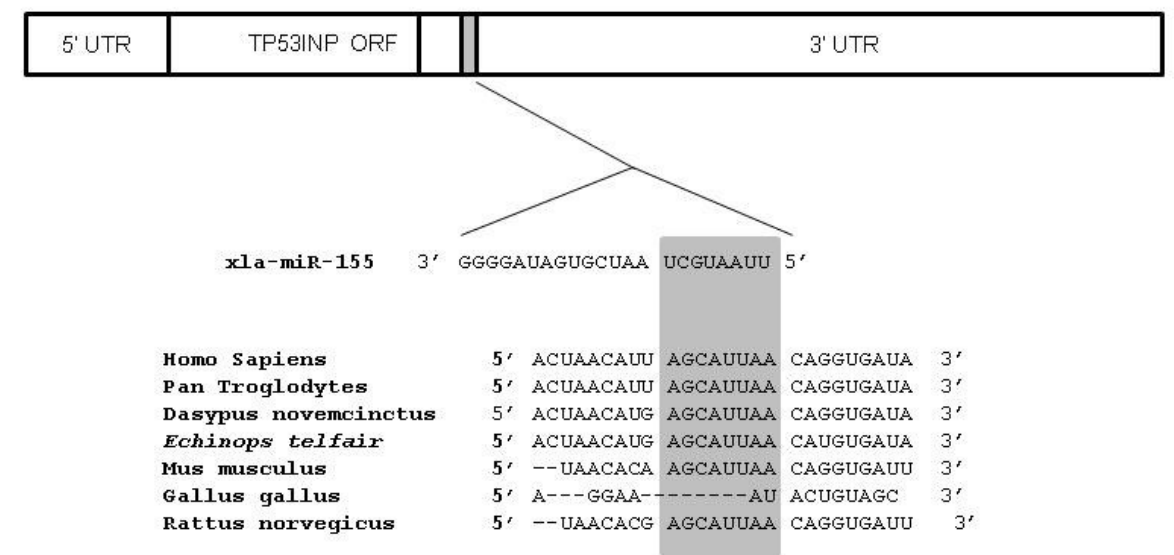

B

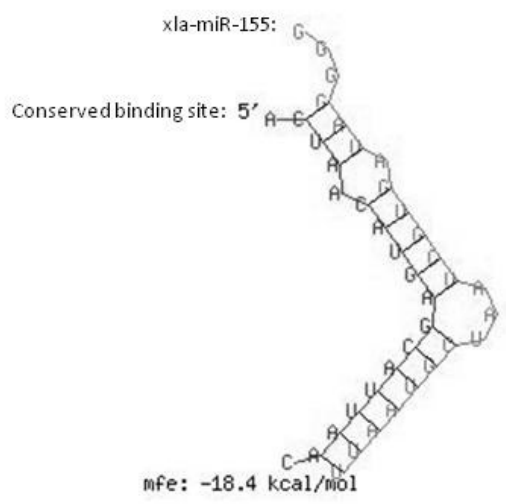

Figure 5.11: (A) Conservation analysis of the mir-155 binding sequence in the 3' UTR of tp53inp. (B) Predicted binding structures of Xenopus specific mir-155 to the 3'UTR of tp53inp with predicted binding energies (generated by RNAhybrid). 


\section{References}

Adams, J.M. and S. Cory. 1998. The Bcl-2 protein family: arbiters of cell survival. Science. 281(5381): 1322-6.

Ageilan, R.I., Calin, G.A. and C.M. Croce. 2010. miR-15a and miR-16-1 in cancer: discovery, function and future perspectives. Cell Death Differ. 17(2): 215-220.

Abramyan, J., Badenhorst, D., Biggar, K.K., Borchert, G.M., Botka, C.W., Bowden, R.M., Braun, E.L., Bronikowski, A.M., Bruneau, B.G., Buck, L.T., Capel, B., Castoe, T.A., Czerwinski, M., Delehaunty, K.D., Edwards, S.V., Fronick, C.C., Fujita, M.K., Fulton, L., Graves, T.A., Green, R.E., Haerty, W., Hariharan, R., Hillier, L.H., Holloway, A.K., Janes, D., Janzen, F.J.,Kandoth, C., Kong, L., de Koning, J., Li, Y., Literman, R., Mardis, E.R., McGaugh, S.E., Minx, P., Mork, L., Laughlin, M., Paitz, R.T., Pollock, D.D., Ponting, C.P., Radhakrishnan, S., Raney, B.J., Richman, J.M., St John, J., Schwartz, T., Sethuraman, A., Shaffer, B., Shedlock, A.M., Spinks, P.Q., Storey, K.B., Thane, N., Thomson, R.C., Valenzuela, N., Vinar, T., Warren, D.E., Warren, W.C., Wilson, R.K., Zimmerman, L.M., Hernandez, O. and C.T. Amemiya. 2013. The western painted turtle genome, a model for the evolution of extreme physiological adaptations in a slowly evolving lineage. Genome Biol. 14(3):R28.

Asangani, I.A., Rasheed, S.A., Nikolova, D.A., Leupold, J.H., Colburn, N.H., Post, S. and H. Allgayer. 2008. MicroRNA-21 (miR-21) post-transcriptionally downregulates tumor suppressor Pdcd4 and stimulates invasion, intravasation and metastasis in colorectal cancer. Oncogene. 27(15): 2128-2136.

Bartel, D.P. 2009. MicroRNAs: target recognition and regulatory functions. Cell. 136: 215-233.

Biggar, K.K. and K.B. Storey. 2012. Evidence for cell cycle suppression and microRNA regulation of cyclin D1 during anoxia exposure in turtles. Cell Cycle 11(9): 1705-1713.

Biggar, K.K. and K.B. Storey. 2011. The emerging roles of microRNAs in the molecular responses of metabolic rate depression. J Mol Cell Biol. 3(3):167-175.

Biggar, K.K., Dubuc, A. and K.B. Storey. 2009. MicroRNA regulation below zero: differential expression of miRNA-21 and miRNA-16 during freezing in wood frogs. Cryobiology. 59(3): 317-221.

Cai, Y. Yu, X., Hu, S. and J. Yu. 2009. A brief review on the mechanisms of miRNA regulation. Genom Proteom Bioinform. 7(4):147-54.

Cimmino, A., Calin, G.A., Fabbri, M., Iorio, M.V., Ferracin, M., Shimizu, M., Wojcik, S.E., Aqeilan, R.I., Zupo, S., Dono, M., Rassenti, L., Alder, H., Volinia, S., Liu, C.G., 
Kipps, T.J., Negrini, M and C.M. Croce. 2005. miR-15 and miR-16 induce apoptosis by targeting BCL2. Proc Natl Acad Sci U S A. 102(39):13944-13949.

Cramp, R.L., Franklin, C.E. and E.A. Meyer. 2005. The impact of prolonged fasting during aestivation on the structure of the small intestine in the green-striped burrowing frog, Cyclorana alboguttata. Acta Zoologica. 86(1): 13-24.

Croce, C.M. 2009. Causes and consequences of microRNA dysregulation in cancer. Nature Rev. 10: 704-714.

Dupont-Versteegden, E.E. 2005. Apoptosis in muscle atrophy: relevance to sarcopenia. Exp. Gerontol. 40(6): 473-81.

Earnshaw, W.C., Martins, L.M. and S.H. Kaufmann. 1999. Mammalian caspases: structure, activation, substrates, and functions during apoptosis. Annu. Rev. Biochem. 68: $383-424$.

Gironella, M. Seux, M., Xie, M.J., Cano, C., Tomasini, R., Gommeaux, J., Garcia, S., Nowak, J., Yeung, M.L., Jeang, K.T., Chaix, A., Fazil, L., Wang, Q., Rocchi, P., Russo., A., Gleave, M., Dogorn, J.C., Iovanna, J.L., Carrier, A., Pebusque, M.J. and N.J. Dusetti. 2007. Tumor protein 53-induced nuclear protein 1 expression is repressed by miR-155, and its restoration inhibits pancreatic tumor development. Proc Natl Acad Sci U S A. 104(41): 16170-16175.

Guo C.J., Pan, Q., Li, D.G., Sun, H., and B.W. Liu. 2009. miR-15b and miR-16 are implicated in activation of the rat hepatic stellate cell: An essential role for apoptosis. J Hepatol. 50(4):766-78.

Hammond, S.M. 2006. MicroRNAs as oncogenes. Curr Opin Genet Dev. 16(1):4-9.

Hay, N. and N. Sonenberg. 2004. Upstream and downstream of mTOR. Genes Dev. 18(16): 1926-1945

Hwang H.W. and J.T. Mendell. 2006. MicroRNAs in cell proliferation, cell death, and tumorigenesis. Br J Cancer. 94: 776-780.

Hudson, N.J. and C.E Franklin. 2002. Effect of aestivation on muscle characteristics and locomotor performance in the green-striped burrowing frog, Cyclorana alboguttata. J. Comp. Physiol. B. 172(2): 177-82.

Earnshaw, W.C., Martins, L.M. and S.H. Kaufmann. 1999. Mammalian caspases: structure, activation, substrates, and functions during apoptosis. Annu. Rev. Biochem. 68:

Iorio, M.V., Ferracin, M., Liu, C.G., Veronese, A., Spizzo, A., Spizzo, R., Sabbioni, S., Magri, E., Pedriali, M., Fabbri, M., Campiglio, M., Menard, S., Palazzo, J.P., Rosenberg, A., Musiani, P., Volinia, S., Nenci, I., Calin, G.A., Querzoli, P., Negrini, M. and C.M. 
Croce. 2005. MicroRNA gene expression deregulation in human breast cancer. Cancer Res. 15: 65(16): 7065-70.

Jovanovic, M. and M.O. Hengartner. 2006. miRNAs and apoptosis: RNAs to die for. Oncogene, 25, 6176-6187.

Krichevsky, A.M and G. Gabriely. 2009. miR-21: a small multi-faceted RNA.J Cell Mol Med. 13(1): 39-53.

Michels, J., Johnson, P.W.M. and G. Packham. 2005. Mcl-1. Int. J. Biochem. Cell Biol. 37: 267-271.

Morin, P., Dubuc, A., and K.B. Storey. 2008. Differential expression of microRNA species in organs of hibernating ground squirrels: a role in translational suppression during torpor. Biochim Biophys Acta - Gene Reg Mech. 1779: 628-633.

O’Connor, L., Strasser, A., O’Reilly, L.A., Hausmann, G., Adams, J.M., Cory, S. and D.C. Huang. 1998. Bim: a novel member of the Bcl-2 family that promotes apoptosis. EMBO J. 17(2): 384-395.

Olive, V., Bennett, M.J., Walker, J.C., Ma, C., Jiang, I., Cordon-Cardo, C., Li., Q., Lowe, S.W., Hannon, G.J. and L. He. 2009. miR-19 is a key oncogenic component of mir-17-92. Genes Dev. 23(24): 2839-2849.

Olive, V., Jiang, I. and L. He. 2010. mir-17-92, a cluster of miRNAs in the midst of the cancer network. Int J Biochem Cell Biol. 42(8): 1348-1354.

Seike, M., Goto, A., Okano, T., Bowman, E.D., Schetter, A.J., Horikawa, I., Mathe, E.A., Jen, J., Yang, P., Sugimura, H., Gemma, A., Kodoh, S., Croce, C.M. and C.C. Harris. 2009. MiR-21 is an EGFR-regulated anti-apoptotic factor in lung cancer in neversmokers. Proc Natl Acad Sci U S A. 106(29): 12085-12090.

Si, M.L, Zhu, S., Wu, H., Lu., Z., Wu, F. and Y.Y. Mo. 2007. miR-21-mediated tumor growth. Oncogene. 26(19): 2799-2803.

Singh, J. and J. Nagaraju. 2008 In silico prediction and characterization of microRNAs from red flour beetle (Tribolium castaneum). Insect Mol Biol. 17(4): 427-436.

Storey, K.B. and J.M. Storey. 2010. Metabolic regulation and gene expression during aestivation. In: Aestivation: Molecular and Physiological Aspects (Navas, C.A. and Carvalho, J.E., eds), Progress in Molecular and Subcellular Biology, Springer, Heidelberg, Vol. 49, pp. 25-45. 
Storey, K.B. and J.M. Storey. 2011. Strategies of molecular adaptation to climate change: the challenges for amphibians and reptiles. In: Temperature Adaptation in a Changing Climate (Storey, K.B. and Tannino, K., eds), CABI Publishers: Wallingford, UK.

Su, H., Yang, J.R., Xu, T., Huang, J., Xu, L., Yuan, Y. and S.M. Zhuang. 2009. MicroRNA-101, down-regulated in hepatocellular carcinoma, promotes apoptosis and suppresses tumorigenicity. Cancer Res. 69(3):1135-42.

Subramanian, S. and C.J. Steer. 2010. MicroRNAs as gatekeepers of apoptosis. J Cell Physiol. 223(2): 289-98.

Veleri, N. Gasparini, P., Fabbri, M., Broconi, C., Veronese, A., Lovat, F., Adair, B., Vannini, I., Fanini, F., Bottoni, A., Costinean, S., Sandhu, S.K., Nuovo, G.J., Alder, H., Gafa, R., Calore, F., Ferracin, M., Lanza, G., Volinia, S., Negrini, M., Mcllhatton, M.A., Amadori, D., Fishel, R. and C.M. Croce. 2010. Modulation of mismatch repair and genomic stability by miR-155. Proc Natl Acad Sci U S A. 107(15): 6982-6987.

Xia, L., Zhang, D., Du, R., Pan, Y., Zhao, L., Sun, S., Hong, L., Liu, J. and D. Fan. 2008. miR-15b and miR-16 modulate multidrug resistance by targeting BCL2 in human gastric cancer cells. Int J Cancer. 123(2): 372-279.

Yanaihara, N., Caplen, N., Bowman, E., Seike, M., Kumamoto, K., Yi., M., Stephens, R.M., Okamoto, A., Yokota, J., Tanaka, T., Calin, G.A., Liu, C.G., Croce, C.M., and C.C. Harris. 2006. Unique microRNA molecular profiles in lung cancer diagnosis and prognosis. Cancer Cell. 9(3): 189-198.

Zhang, Z., Li, Z., Gao, C., Chen, P., Chen, J., Liu, W., Xiao, S. and H. Lu. 2008. miR-21 plays a pivotal role in gastric cancer pathogenesis and progression. Lab. Invest. 17(4) 427-436.

Zimmermann, K.C. and D.R. Green. 2001. How cells die: apoptosis pathways. J. Allergy Clin. Immunol. 108(4): S99-103. 


\section{Chapter 6:}

\section{General Discussion}




\section{Background}

This thesis analysed the role of anti-apoptotic pathways which act to preserve tissues and minimize atrophy during medium and high levels of dehydration in X. laevis. Xenopus is found in sub-Saharan Africa where it lives in swamps, lakes, rivers and manmade ponds/wells. The frog is almost exclusively aquatic although during the dry season it has to face the drying up of its habitat and adapt by entering a state of dormancy called estivation (Alexander and Bellerby, 1938). During this period, adapted animals strongly suppress their metabolic rate while conserving energy by reprioritizing many physiological and biochemical processes and activating a variety of cell preservation strategies that protect the animal during long-term dormancy (Storey and Storey, 2010; 2012). In addition, in estivating animals, this period is usually characterized by substantial dehydration stress, with Xenopus being able to endure the loss of up to about $30 \%$ of its total body water. Aestivators typically display high desiccation tolerance achieved through physiological changes as well as the upregulation of metabolic pathways critical for survival (Romspert, 1975). Deeper understanding of the processes taking place during Xenopus dehydration will provide an insight into the current understanding of estivation as a whole.

Estivating animals spend the dry season immobile in their burrows with little or no access to food and water, and typically using only "on board" stores of metabolic fuels and water to support survival. Muscle and digestive organ atrophy caused by disuse becomes a great concern that needs to be addressed. Frogs and toads usually mate immediately upon emerging from their estivation spot, and so they must be able to contract their skeletal muscles to be able to move and use their digestive organs to absorb 
important nutrients (Hudson and Franklin, 2002). The biochemical pathways that are activated during estivation that preserve tissue integrity and reduce muscle and digestive organ atrophy during dormancy are not well understood. This study analysed the differential expression of anti-apoptotic mechanisms in response to dehydration in skeletal muscle and liver of $X$. laevis.

\section{Activation of $N F-\kappa B$ and STAT}

Chapter 3 evaluated the responses of the NF- $\mathrm{kB}$ pathway in X. laevis liver and muscle during their exposure to medium and high levels of dehydration. NF- $\mathrm{KB}$ is a known regulator of the human immune response that has recently been identified as also activated in response to other stresses such as hemorrhagic shock, oxidative stress, environmental hazards, etc. (Paul, 1999). It has also been described as a major marker of a number of human cancers since its target genes include those with vital roles in cell survival (Nishikori, 2005). Under normal conditions, NF-kB exists as a dimer (most commonly composed of p-50 and p-65 protein) bound by its inhibitor, IKB $\alpha$. In response

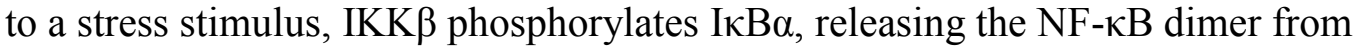
inhibitory control and allowing it to translocate into the nucleus. This chapter evaluated the relative expression of the p-50 and p-65 subunits along with their phosphorylated forms as well as the expression of anti-apoptotic downstream targets, Bcl-xL and c-IAP (Senftleben et al, 2001).

The results presented in Chapter 3 suggested that during dehydration, NF- $\mathrm{kB}$ activity is increased through the phosphorylation of its subunits, p-50 and p- 65 , and the degradation of its inhibitor I $\mathrm{I} B \alpha$. In addition, evidence showed an increased presence of 
phosphorylated NF- $\mathrm{BB}$ subunits in the nucleus under dehydration stress conditions compared to controls. The expression levels of the two anti-apoptotic downstream targets examined, c-IAP1 and Bcl-xL, were also elevated under dehydration conditions. No evidence for the activation of the NF-kB pathway was found in skeletal muscle, however similar to liver, the majority of the anti-apoptotic downstream targets evaluated were found to be elevated. Since activation of the anti-apoptotic targets in skeletal muscle appeared to be independent of NF- $\mathrm{KB}$ activation, Chapter 4 addressed the possibility of the activation of an alternative mechanism of anti-apoptotic control and evaluated the STAT pathway.

The STAT transcription factor family consists of seven members, STATs 1, 2, 3, 4, 5A, 5B and 6), that play important roles in the immune response, $\mathrm{T}$ helper cell differentiation, cell cycle progression, as well as cell growth and survival (Imada and Leonard, 2000). Under conditions of stress, the pathway is initiated by JAK enzyme mediated phosphorylation of STATs which proceed to form either homodimers or heterodimers followed by a translocation into the nucleus (Alvarez and Frank, 2004). The two main STAT proteins that activate anti-apoptotic genes in response to a stimulus are STAT3 and STAT5 and these were found to be differentially regulated in the liver and skeletal muscle of dehydrated Xenopus. Figures 6.1-6.8 present a summary of the data gathered in Chapters 3-5 in order to provide a clear summary of the relationships between the examined pathways.

This chapter also provided a potential explanation for the activation of Bcl-xL and c-IAP1 in the muscle in the absence of NF- $\mathrm{kB}$ activation, since the data provided evidence for the activation of the STAT5 transcription factor. Experiments using nuclear 
extracts in addition to DNA binding assays showed an increased abundance of STAT5 (Tyr 694) in the nucleus during dehydration stress. In addition to the already examined Bcl-xL and c-IAP1 downstream targets, other anti-apoptotic target of STAT5 include Bcl-2 and Mcl-1 (Figure 6.1-6.4). Figures 6.3 and 6.4 show the combined expression levels of all the examined anti-apoptotic targets in the skeletal muscle of Xenopus. Bcl$\mathrm{xL}, \mathrm{c}-\mathrm{IAP} 1$ and Bcl-2 all showed evidence of relative increases in both mRNA transcripts and protein products occurring in either both dehydration conditions or limited to high dehydration.

In contrast, in the liver tissue, there was evidence of STAT3 activation and nuclear localization in response to dehydration. Figures 6.1 and 6.2 showed the relative mRNA and protein levels of all the anti-apoptotic targets of STAT3 including c-IAP1, Bcl-xL, Bcl-2, and Mcl-1. While mRNA levels of c-IAP increased in high dehydration, relative levels of Bcl-xL increased in both dehydration conditions. Similar to the skeletal muscle tissue, relative protein expression levels of c-IAP1, Bcl-xL, and Bcl-2 were increased in liver under either one or both of the dehydration conditions. Although the NF- $\mathrm{BB}$ and STAT pathways were alternatively activated in liver and skeletal muscle, there is strong evidence to suggest that anti-apoptotic pathways are activated during Xenopus dehydration by the activation of transcription factor pathways and the upregulation of their anti-apoptotic downstream targets. The active regulation of these genes during dehydration is indicative of their vital role during this time of stress in Xenopus.

STAT3 and NF-kB may competitively bind to the promoter region of their shared downstream targets. Both of these transcription factors are activated during the acute 
inflammatory reaction and regulate the expression of several acute phase proteins such as $\alpha_{2}$-macroglobulin $(\alpha 2 \mathrm{MG})$. A recent paper reported that STAT3 and NF- $\kappa B$ competed for the binding of a labeled probe derived from $\alpha 2 \mathrm{MG}$ gene promoter and proposed several other genes that can be subject to competitive regulation by STAT3/NF-kB (Zhang and Fuller, 1997). Similar competitive binding may be taking place in Xenopus liver where both STAT3 and NF-kB are activated during dehydration stress. Further studies are required to analyse which transcription factor is responsible for the activation of which downstream target.

\section{MiRNA regulation}

It is clear from the results of this study that tissues of dehydrated Xenopus actively upregulate the expression of genes whose protein products work to protect their cells from programmed cell death. The active transcription of these genes is at least in part activated by the action of NF- $\kappa$ and the STAT transcription factors. In Chapter 5, another mode of gene regulation under stress conditions was analyzed: regulation at the post-transcriptional level by miRNAs. These small (18-22 nt in length), non-coding RNA transcripts are able to bind to the 3'UTR of their target genes and direct the RNA-induced silencing complex (RISC) to induce mRNA degradation. The fate of the target transcript depends on its base pairing to the miRNA. Perfect complementarity between the miRNA and its target results in cleavage of the mRNA by RISC, whereas imperfect base pairing results in translational repression of the gene via storage in cytoplasmic p-bodies (Subramanian and Steer, 2010). The present study measured the expression of 8 
miRNAs, three pro-apoptotic and five anti-apoptotic, that have recently been found to play vital roles in the regulation of the cell death program.

The anti-apoptotic miRNAs analysed were mir-19b, -21, -92a, -133, and -155. In the liver relative levels of mir-19b significantly increased in the high dehydration condition while relative levels of mir-155 significantly increased in both dehydration conditions. In skeletal muscle, relative levels of mir-21, -92a, -133, and -155 all increased in the high dehydration condition. Interestingly, relative levels of mir-92a and mir-155 first fell in medium dehydration before rising in the high dehydration condition. Overall, the evidence showed that miRNAs with identified anti-apoptotic functions generally rose during high dehydration in Xenopus liver and skeletal muscle.

The three examined pro-apoptotic miRNAs were mir-15a, mir-16 and mir-101. It was found that mir-15a and mir-16 which target Bcl-2 were differentially regulated in the liver and skeletal muscle tissues. Mir-15a significantly increased in high dehydration in the liver tissue and decreased in medium dehydration in the skeletal muscle tissue. In addition, mir-16 significantly decreased in skeletal muscle under high dehydration conditions. Meanwhile, relative levels of Bcl-2 mRNA and protein levels remained constant in the liver tissue and significantly increased in medium and high dehydration in the skeletal muscle tissue.

There is evidence to indicate that $b c l-2$ is at least in part regulated by mir-15a and mir-16 due to the inverse correlation of the levels of these miRNAs with Bcl-2 mRNA transcript and protein product levels (Figure 6.5 and 6.6). In the liver tissue mir-15a significantly increased while $b c l-2$ levels remained unchanged. A possible explanation is that mir-15a regulates $b c l-2$ by binding to the 3 'UTR and storing the transcript in p- 
bodies until a time when it is needed. In response to a certain stimulus, mir-15a releases $b c l-2$ from its inhibitory control allowing it to bind the transcriptional machinery. However, the purpose of the active inhibition of $b c l-2$ in the liver tissue by miRNAs despite activation of $b c l-2$ expression by both NF- $\mathrm{KB}$ and STAT proteins is unclear.

Mir-101 is able to bind to and inhibit the activation of Mcl-1, an important antiapoptotic gene. In this study, mir-101 transcript levels were elevated in both liver and skeletal muscle during the high dehydration condition. Relative transcript levels of $m c l-1$ significantly decreased under both dehydration conditions in the liver while levels rose in the high dehydration condition in skeletal muscle. Relative protein levels remained constant across conditions in both tissues. The picture is less clear in the case of mir-101 control over Mcl-1 than the previously discussed relationship between mir-15a and mir16 with Bcl-2 (Figure 6.7 and 6.8). While an inverse relationship is apparent in the liver tissue, both mir-101 and mcl-1 mRNA rose in the high dehydration condition. As protein levels did not correlate with the elevated mRNA levels, one theory is that the $m c l-1$ gene is actively transcribed in response to the stimulus of dehydration and is subsequently stored in p-bodies in association with mir-101 until it is needed.

Overall, it appears that dehydrated Xenopus liver and skeletal muscle tissues actively resist apoptosis my activating proteins that function to resist the onset of the cell death program. In addition, there was evidence to indicate that regulation of both antiand pro-apoptotic genes takes place at the post-transcriptional level through the action of miRNAs. 


\section{Future research}

The intent of the studies described in this thesis was to further the current understanding of the survival mechanisms utilized by Xenopus to deal with whole body dehydration, an environmental stress that is naturally encountered by this frog during the dry season and is a component of estivation. The results suggest that Xenopus actively resists cell death during dehydration by up-regulating anti-apoptotic pathways, however further experiments are required to obtain a full picture of the relevant cellular pathways.

The progression of the cell death program mainly relies on the action of caspases, a family of enzymes that are responsible for the cleavage and degradation of several intracellular polypeptides that are important to the structural integrity of the cell and components of the DNA repair machinery (Earnshaw et al., 1999). There are two types of caspase proteins, those termed "executer" caspases including caspase $-3,-6$ and -7 that coordinate the events associated with cell death, and those termed the "initiator" caspases including caspase- 8 and -9 that cleave executer caspases to convert them to their active forms (Zimmermann and Green, 2001). A major event in the apoptosis pathway is the release of cytochrome $\mathrm{C}$ into the cytoplasm which joins the apoptotic protease activating factor-1 (Apaf-1), procaspase-9, and dATP to form an apoptosome. This complex cleaves procaspase-3 to its active form thereby initiating the cell death response (Zimmermann and Green, 2001). A survey of the relative expression levels of the initiator and executer caspases along with cytochrome $\mathrm{C}$ and Apaf-1 would provide further insight into the regulation of apoptosis during Xenopus dehydration and would be an important area for study in future research. 
The Bcl-2 family of proteins includes proteins that assist the progression of the cell death program (e.g. Bax, BAD, and BIM), and those that oppose its action (e.g., Bcl-2, Bcl-xL, and Bcl-w). This study focused on the expression levels of Bcl-2 and Bcl-xL but a survey of all 14 of the Bcl-2 family members would be beneficial for the understanding of their roles in stress survival.

Furthermore, a survey of the activity of other transcription factors that can regulate the apoptotic pathway would be beneficial. In particular, three transcription factors are of special interest currently. The PAX3 transcription factor has been recently identified as regulating the transcription of Bcl-xL whereas the FKHR-L1 transcription factor has been found to regulate BIM (Margue et al, 2002; Dijkers et al, 2000). The HIF-1 $\alpha$ transcription factor targets genes that include those that regulate cell proliferation and angiogenesis but can also target two of the pro-apoptotic Bcl-2 family members, BNip3 and BNip3 (Piret et al, 2002).

The present thesis also evaluated the expression of 7 miRNAs that are known regulators of apoptosis and important markers of cancer. However over 30 miRNAs have been identified to date that play vital roles in cell death and survival. Hence, an expanded survey of many other miRNA types, along with their target genes, would be an interesting new direction for continuing research.

Finally, the next challenge for future studies is to examine the apoptotic pathway in other tissues of Xenopus and is response to dehydration. For example, the kidney is responsible for regulating the disposal of soluble waste products and also the recovery of water and nutrients from the kidney filtrate. Water conservation is extremely important during periods of water shortage so it could be predicted that anti-apoptotic mechanisms 
would predominate in kidney in response to dehydration in Xenopus . Other tissues that could provide important information on the involvement of apoptosis in estivation or dehydration tolerance are the brain, a tissue that has to be protected throughout prolonged periods of dehydration stress and/or estivation, the skin that has a major role in water regulation in amphibians, and the heart that must continue to contract and pump blood even while blood volume and viscosity changes dramatically during dehydration.

Xenopus has become a model for dehydration and estivation (metabolic rate depression) research only in recent years and so relatively little is known to date about the cellular processes which are activated and/or deactivated during the course of $X$. laevis estivation and dehydration. This thesis attempted to further the current understanding, but further studies are required to fully evaluate the cellular responses by Xenopus organs to dehydration and the survival mechanisms that are activated. 


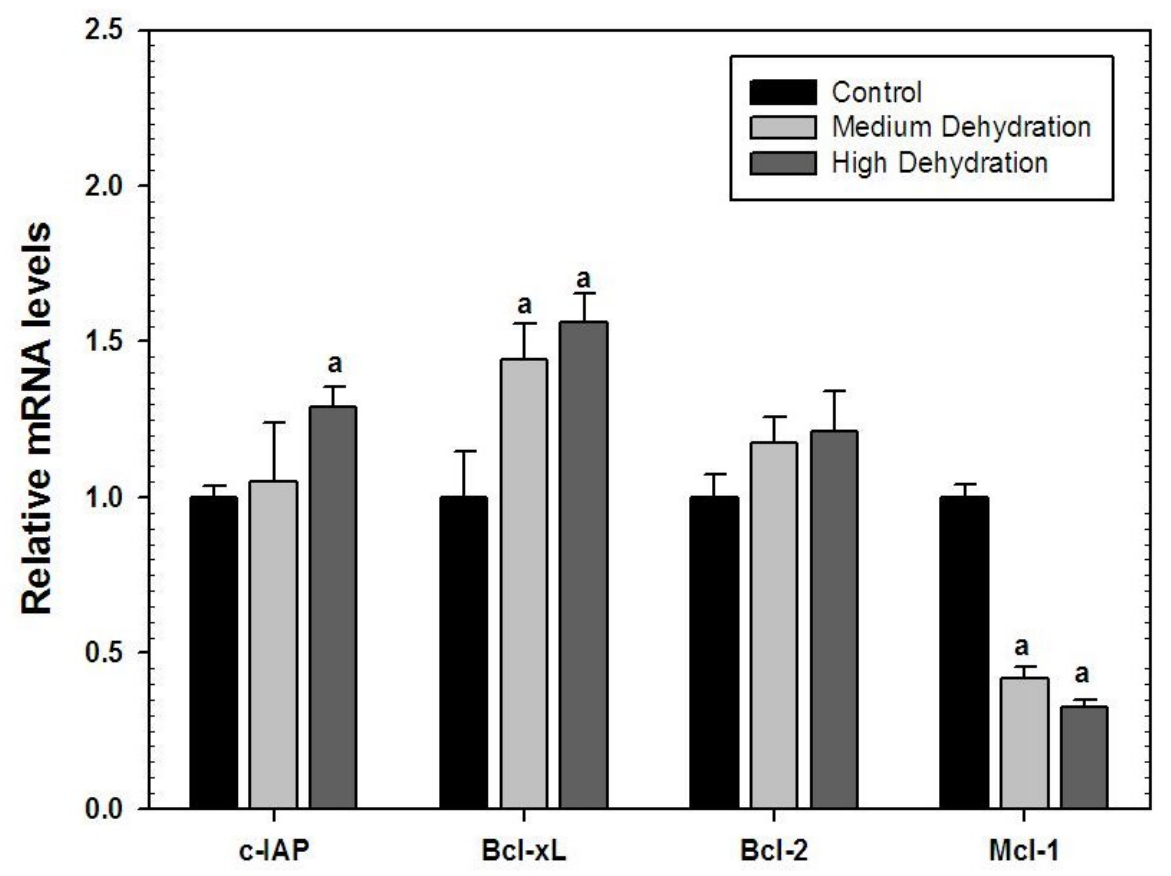

Figure 6.1: Summary figure of the relative changes in mRNA transcript levels of the combined NF- $\kappa$ B and STAT downstream targets; c-IAP, Bcl-xL, bcl-2 and mcl-1 in liver in response to medium and high dehydration of $X$. laevis. Data are means $\pm \mathrm{SEM}$ for $\mathrm{n}=4$ independent trials. "a" - Significantly different from the control values $(P<0.05)$. 


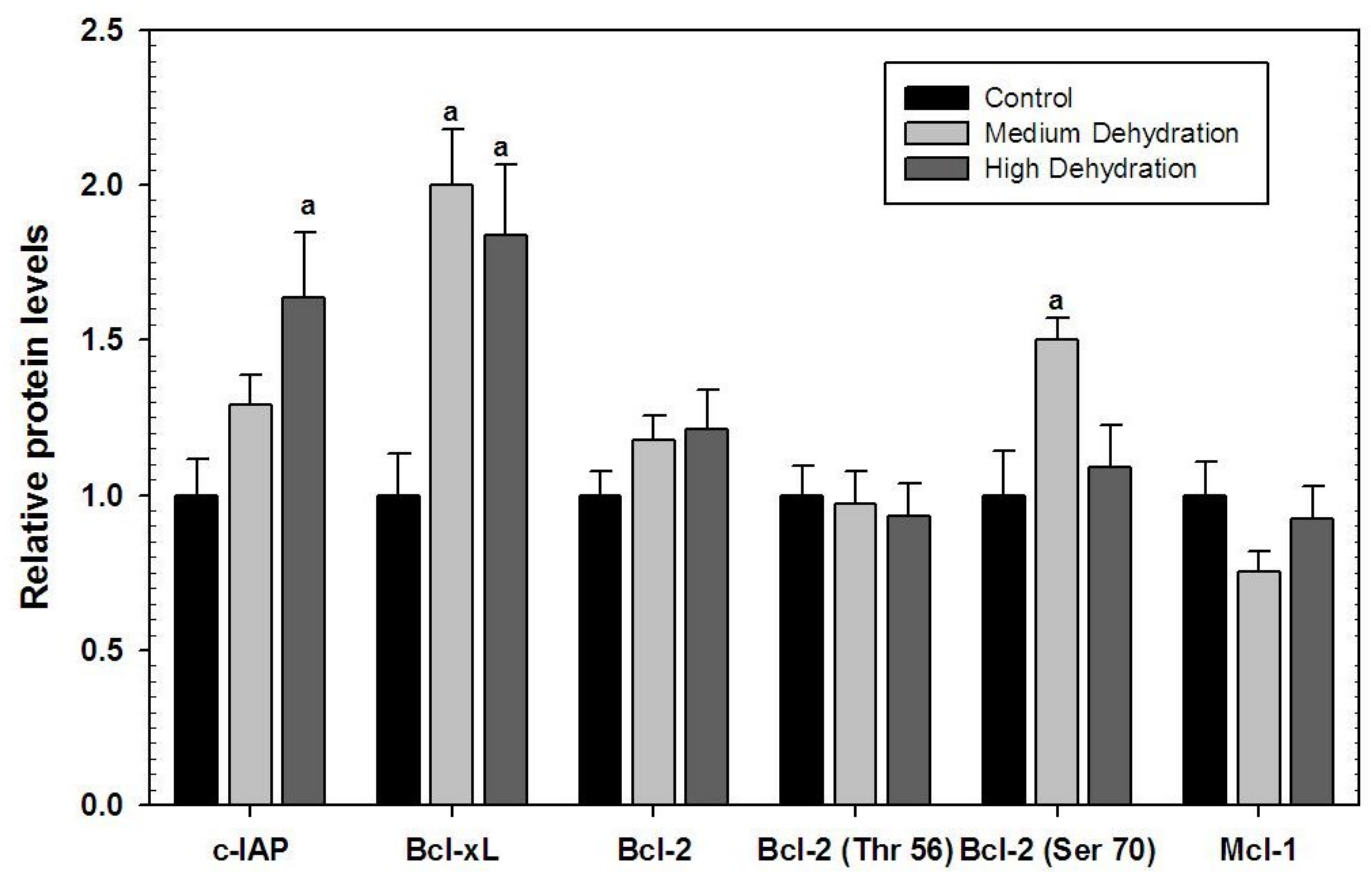

Figure 6.2: Summary figure of the relative changes in protein levels of the combined NF- $\kappa \mathrm{B}$ and STAT downstream targets; c-IAP, Bcl-xL, total bcl-2 in addition to bcl-2 (Thr 56) and bcl-2 (Ser 70) in liver tissues during medium and high dehydration of $X$. laevis. Data are means \pm SEM for $\mathrm{n}=4$ independent trials. "a" - Significantly different from the control values $(P<0.05)$. 


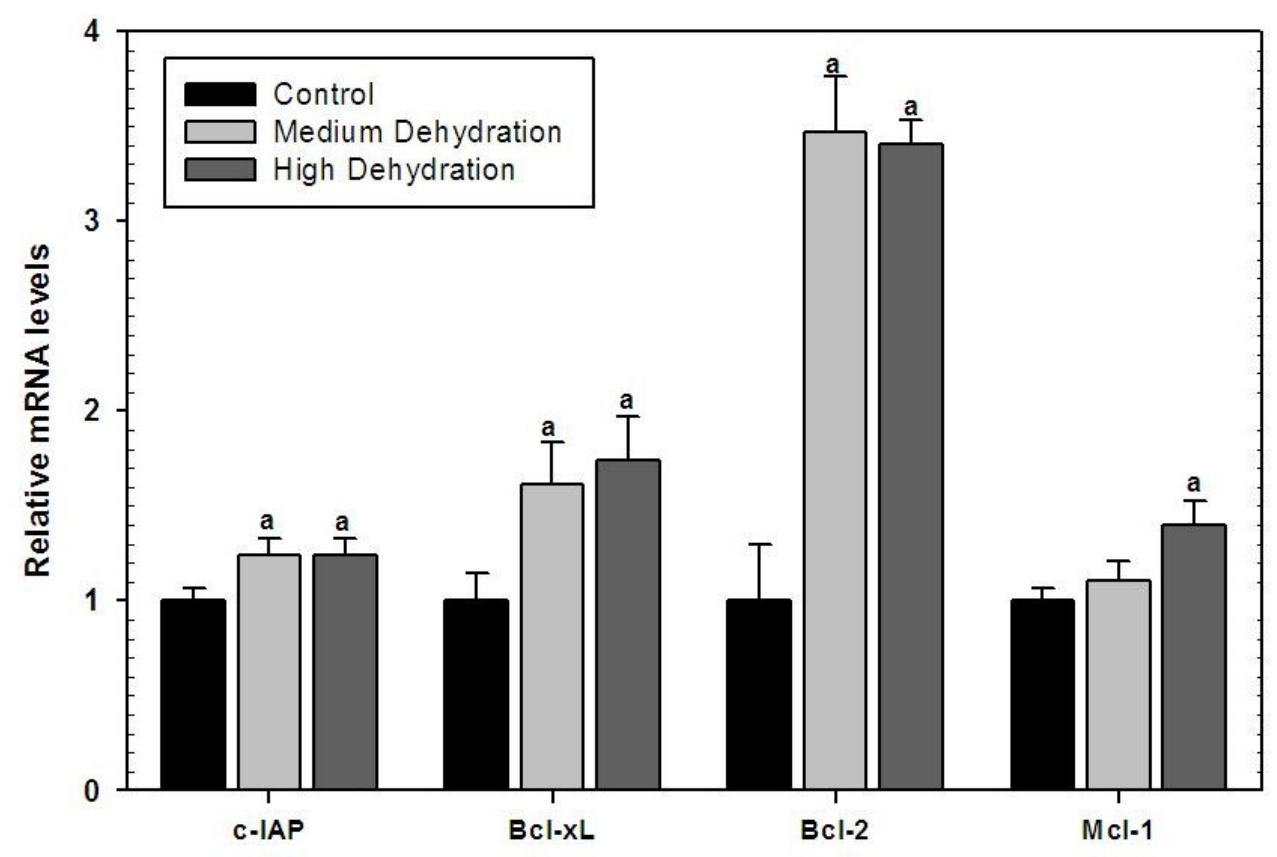

Figure 6.3: Summary figure of the relative changes in mRNA transcript levels of the combined NF- $\kappa$ B and STAT downstream targets; c-IAP, Bcl-xL, bcl-2 and mcl-1 in skeletal muscle in response to medium and high dehydration of $X$. laevis. Data are means \pm SEM for $\mathrm{n}=4$ independent trials. "a" - Significantly different from the control values $(P<0.05)$. 


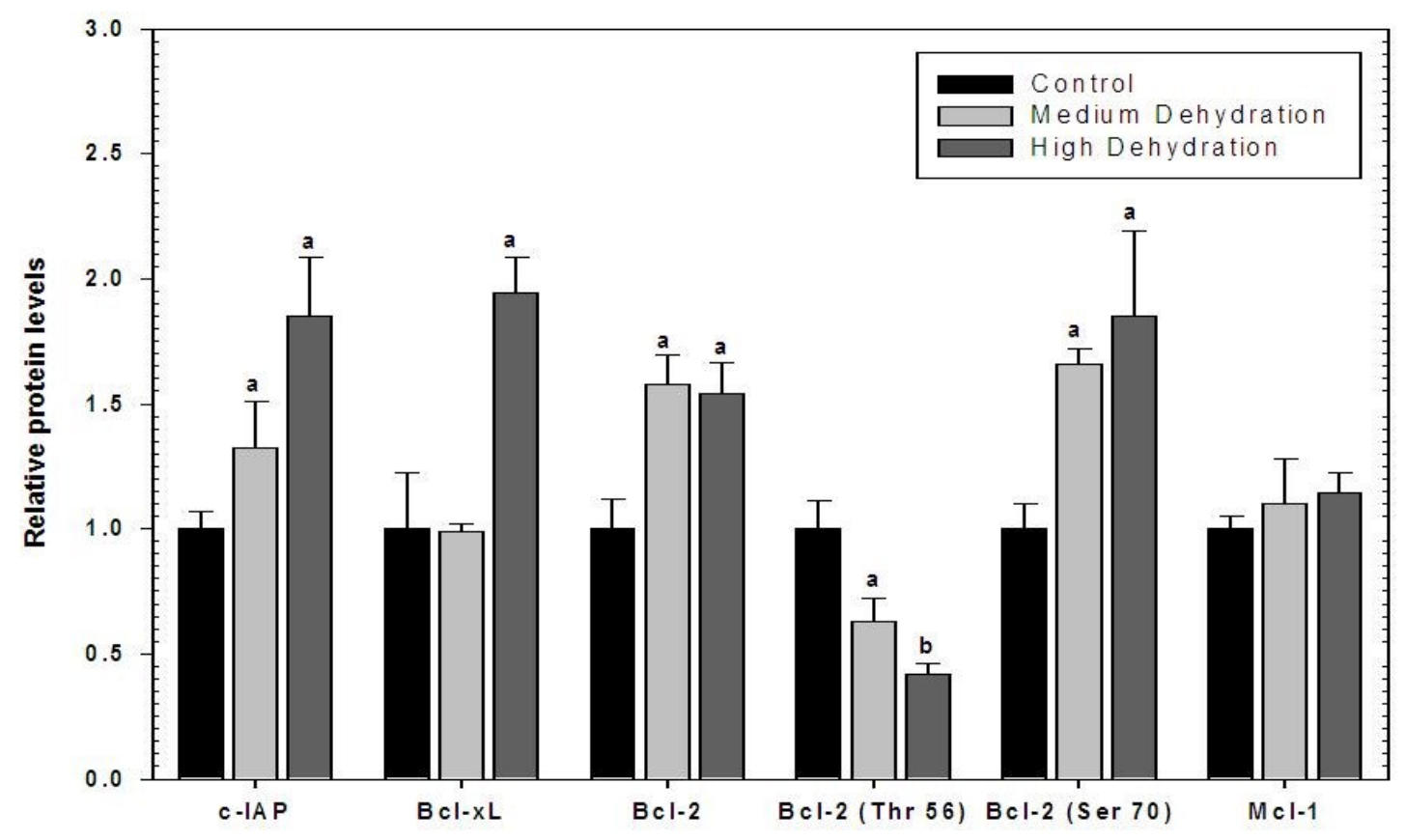

Figure 6.4: Summary figure of the relative changes in protein levels of the combined NF- $\kappa \mathrm{B}$ and STAT downstream targets; c-IAP, Bcl-xL, total bcl-2 in addition to bcl-2 (Thr 56) and bcl-2 (Ser 70) in skeletal muscle tissues during medium and high dehydration of $X$. laevis. Data are means \pm SEM for $n=4$ independent trials. "a" Significantly different from the control values $(P<0.05)$. 


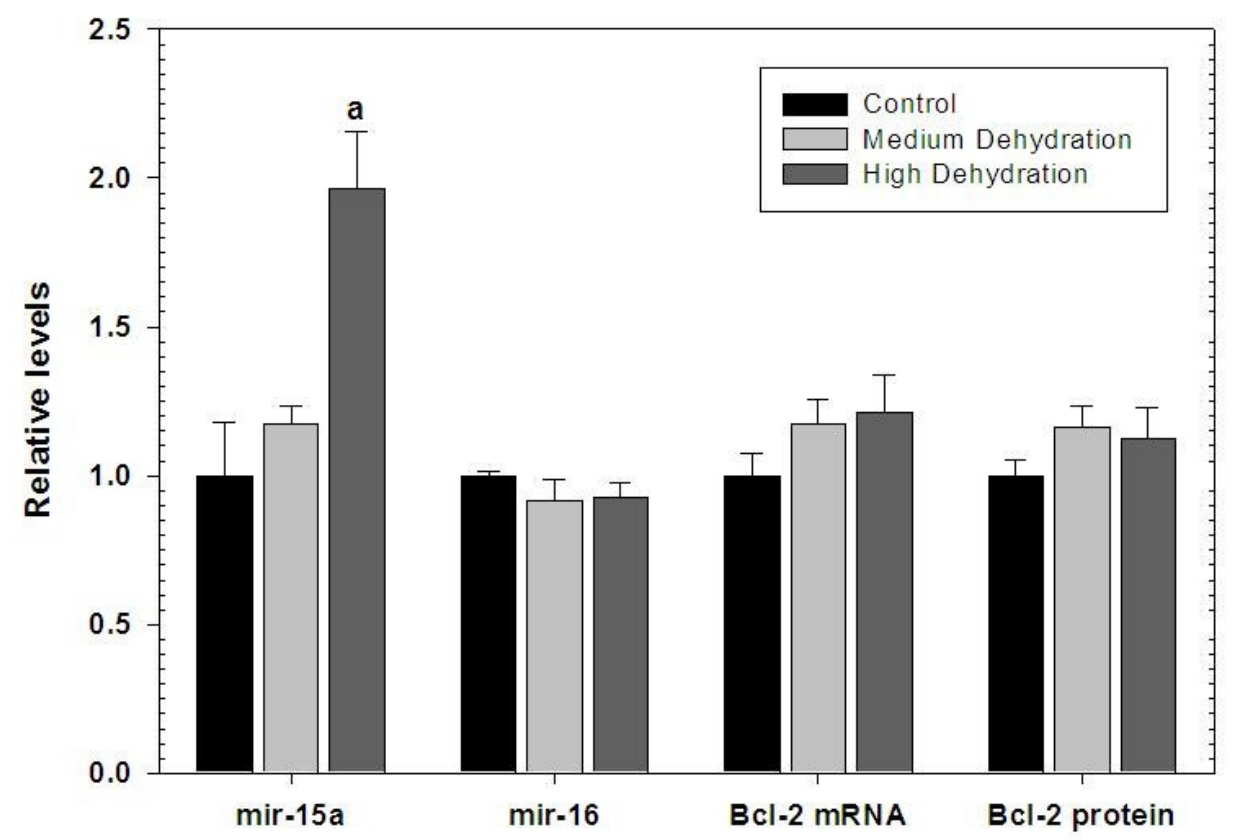

Figure 6.5: Summary figure of the relative changes in the transcript levels of the proapoptotic miRNAs, mir-15a and mir-16, along with the relative changes in mRNA and protein levels of their target gene, $b c l-2$. Figure shows relative changes in liver in response to medium and high dehydration of $X$. laevis. Data are means $\pm \mathrm{SEM}$ for $\mathrm{n}=4$ independent trials. "a" - Significantly different from the control values $(P<0.05)$. 


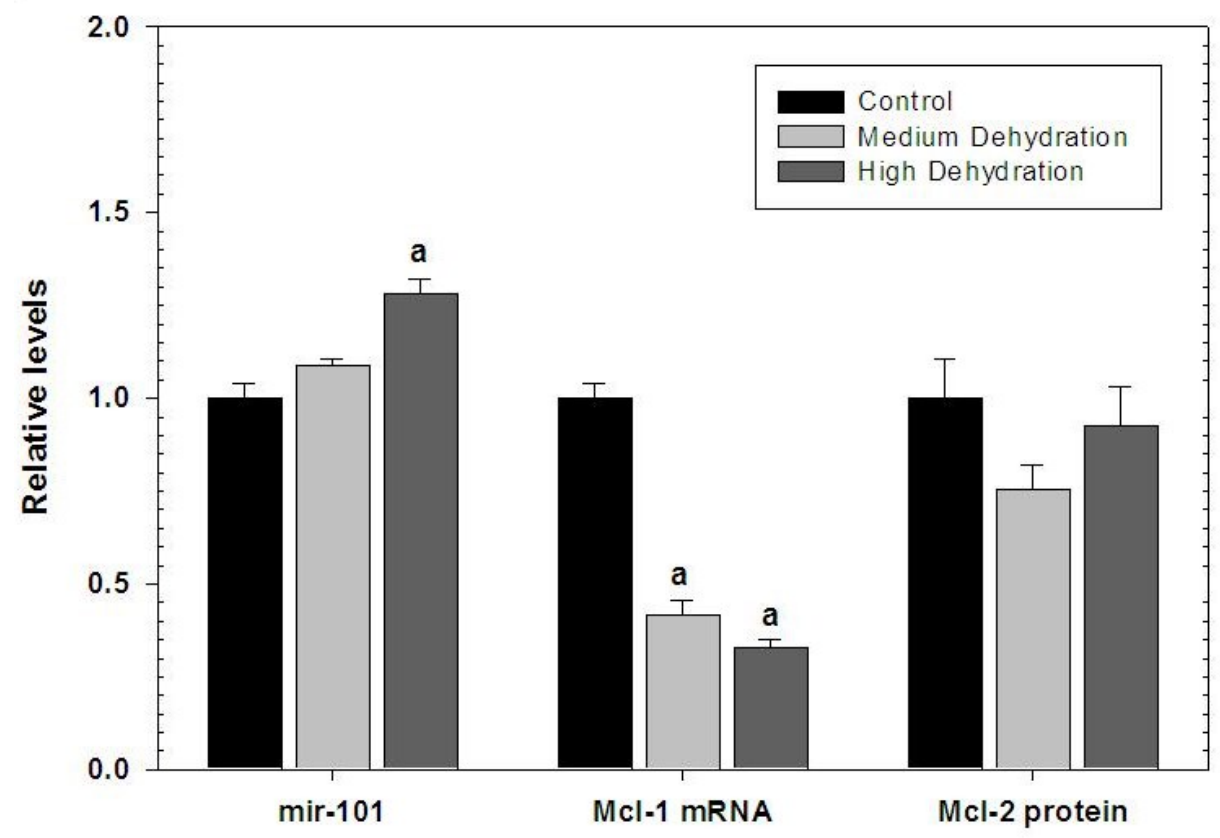

Figure 6.6: Summary figure of the relative changes in the transcript levels of the proapoptotic miRNA, mir-101, along with the relative changes in mRNA and protein levels of its target gene, $m c l-1$. Figure shows relative changes in liver in response to medium and high dehydration of $X$. laevis. Data are means \pm SEM for $\mathrm{n}=4$ independent trials. "a" - Significantly different from the control values $(P<0.05)$. 


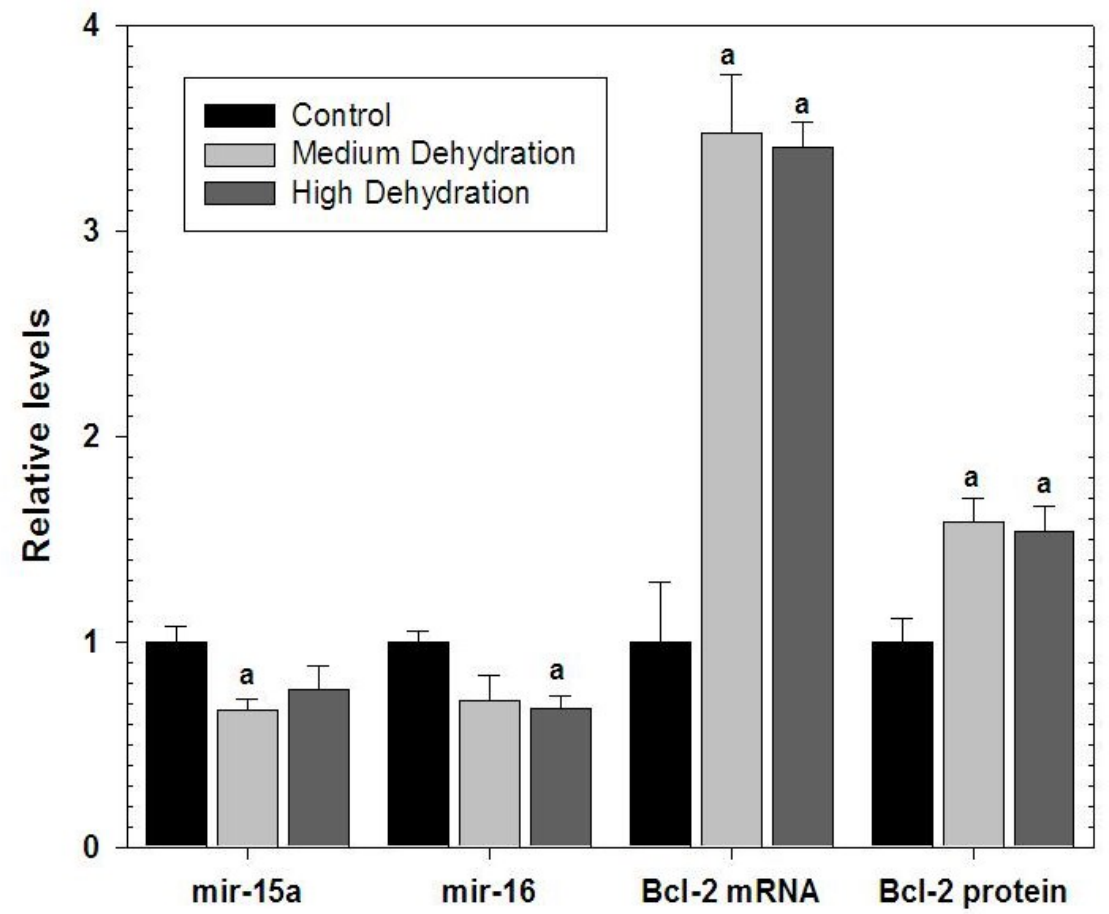

Figure 6.7: Summary figure of the relative changes in the transcript levels of the proapoptotic miRNAs, mir-15a and mir-16, along with the relative changes in mRNA and protein levels of their target gene, $b c l$-2. Figure shows relative changes in skeletal muscle in response to medium and high dehydration of $X$. laevis. Data are means $\pm S E M$ for $n=4$ independent trials. "a" - Significantly different from the control values $(P<0.05)$. 


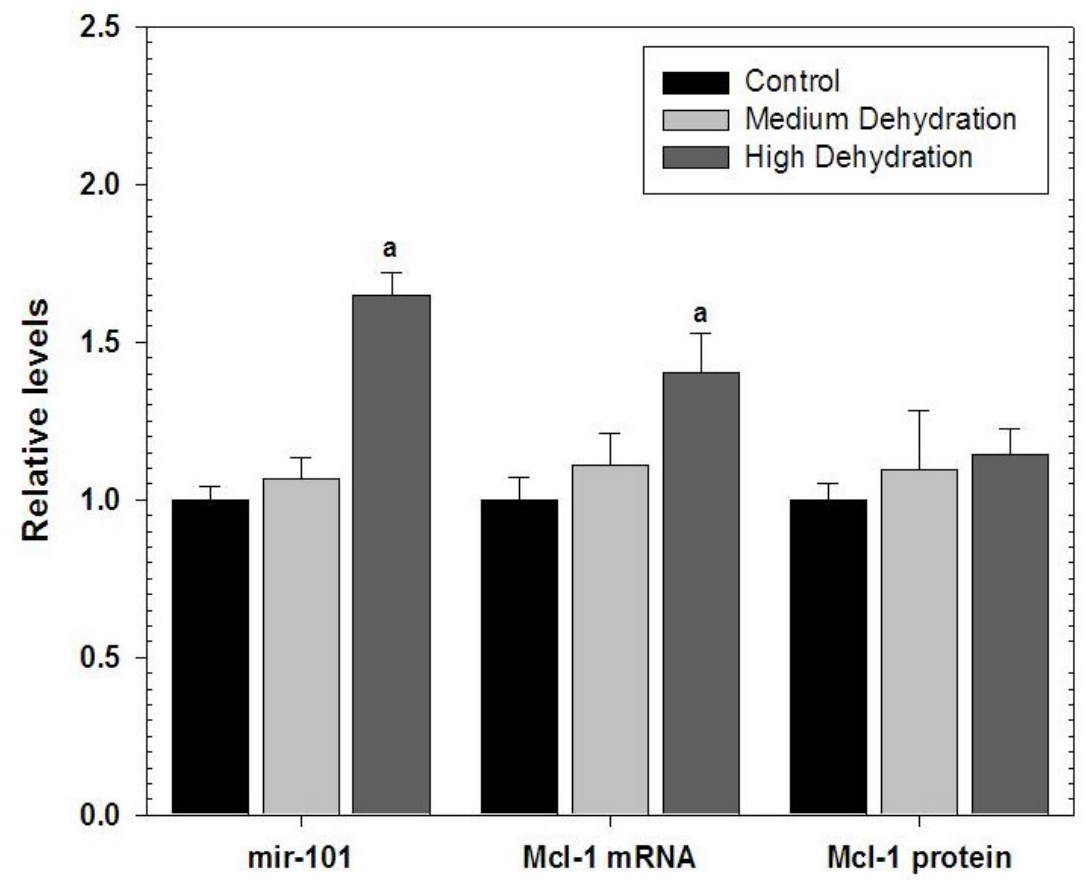

Figure 6.8: Summary figure of the relative changes in the transcript levels of the proapoptotic miRNA, mir-101, along with the relative changes in mRNA and protein levels of its target gene, $m c l-1$. Figure shows relative changes in skeletal muscle in response to medium and high dehydration of $X$. laevis. Data are means $\pm \mathrm{SEM}$ for $\mathrm{n}=4$ independent trials. "a" - Significantly different from the control values $(P<0.05)$. 


\section{References}

Alexander S.S. and C.W. Bellerby. 1938. Experimental studies on the sexual cycle of the South African clawed toad (Xenopus laevis). J. Exp. Biol. 15: 74-81.

Alvarez, J.V. and D.A. Frank. 2004. Genome-wide analysis of STAT target genes: elucidating the mechanism of STAT-mediated oncogenesis. Cancer Biol Ther. 3(11): 1045-50.

Dijkers, P.F., Medema, R.H., Lammers, J.W., Koenderman, L. and P.J. Coffer. 2000. Expression of the pro-apoptotic Bcl-2 family member Bim is regulated by the forkhead transcription factor FKHR-L1. Curr Biol. 10(19):1201-4.

Earnshaw, W.C., Martins, L.M. and S.H. Kaufmann. 1999. Mammalian caspases: structure, activation, substrates, and functions during apoptosis. Annu. Rev. Biochem. 68: W.J. Leonard. 2000. The Jak-STAT pathway. Mol. Immunol. 37: 1-11.

Hudson, N.J. and C.E Franklin. 2002. Effect of aestivation on muscle characteristics and locomotor performance in the green-striped burrowing frog, Cyclorana alboguttata. J. Comp. Physiol. B. 172(2): 177-82.

Margue, C.M., Benasconi, M, Barr, F.G. and B.W. Schafer. 2000. Transcriptional modulation of the anti-apoptotic protein BCL-XL by the paired box transcription factors PAX3 and PAX3/FKHR. Oncogene. 19(25): 2921-2929.

Nishikori, M. 2005. Classical and alternative NF-kB activation pathways and their roles in lymphoid malignancies. J. Clin. Exp. Hematopathol. 45(1): 15-24.

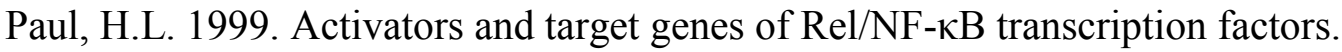
Oncogene 18: 6853- 6866.

Piret, J.P., Mottet, D., Raes, M. and C. Michiels. 2002. Is HIF-1alpha a pro- or an antiapoptotic protein? Biochem Pharmacol. 64(5-6):889-892.

Romspert, A.P. 1975. Osmoregulation of the African clawed frog, Xenopus laevis, in hypersaline media. Comp. Biochem. Physiol. A. 54: 207-210

Senftleben, U., Cao Y., Xiao G., Greten F.R., Krähn G., Bonizzi G., Chen Y., Hu Y., Fong A., Sun S.C., and M. Karin. 2001. Activation by IKKalpha of a second, evolutionary conserved, NF-kappa B signaling pathway. Science. 293(5534): 1495-9

Storey, K.B. and J.M. Storey. 2010. Metabolic regulation and gene expression during aestivation. In: Aestivation: Molecular and Physiological Aspects (Navas, C.A. and Carvalho, J.E., eds), Progress in Molecular and Subcellular Biology, Springer, Heidelberg, Vol. 49, pp. 25-45. 
Storey K.B and J.M Storey. 2012. Aestivation: signaling and hypometabolism. J. Exp. Biol. 215(Pt 9): 1425-33.

Subramanian, S. and C.J. Steer. 2010. MicroRNAs as gatekeepers of apoptosis. J Cell Physiol. 223(2): 289-98.

Zhang, Z. and G.M. Fuller. 1997. The competitive binding of STAT3 and NF-kappaB on an overlapping DNA binding site. Biochem Biophys Res Commun. 237(1): 90-4.

Zimmermann, K.C. and D.R. Green. 2001. How cells die: apoptosis pathways. J. Allergy Clin. Immunol. 108(4): S99-103. 


\section{$\underline{\text { Appendix A }}$}
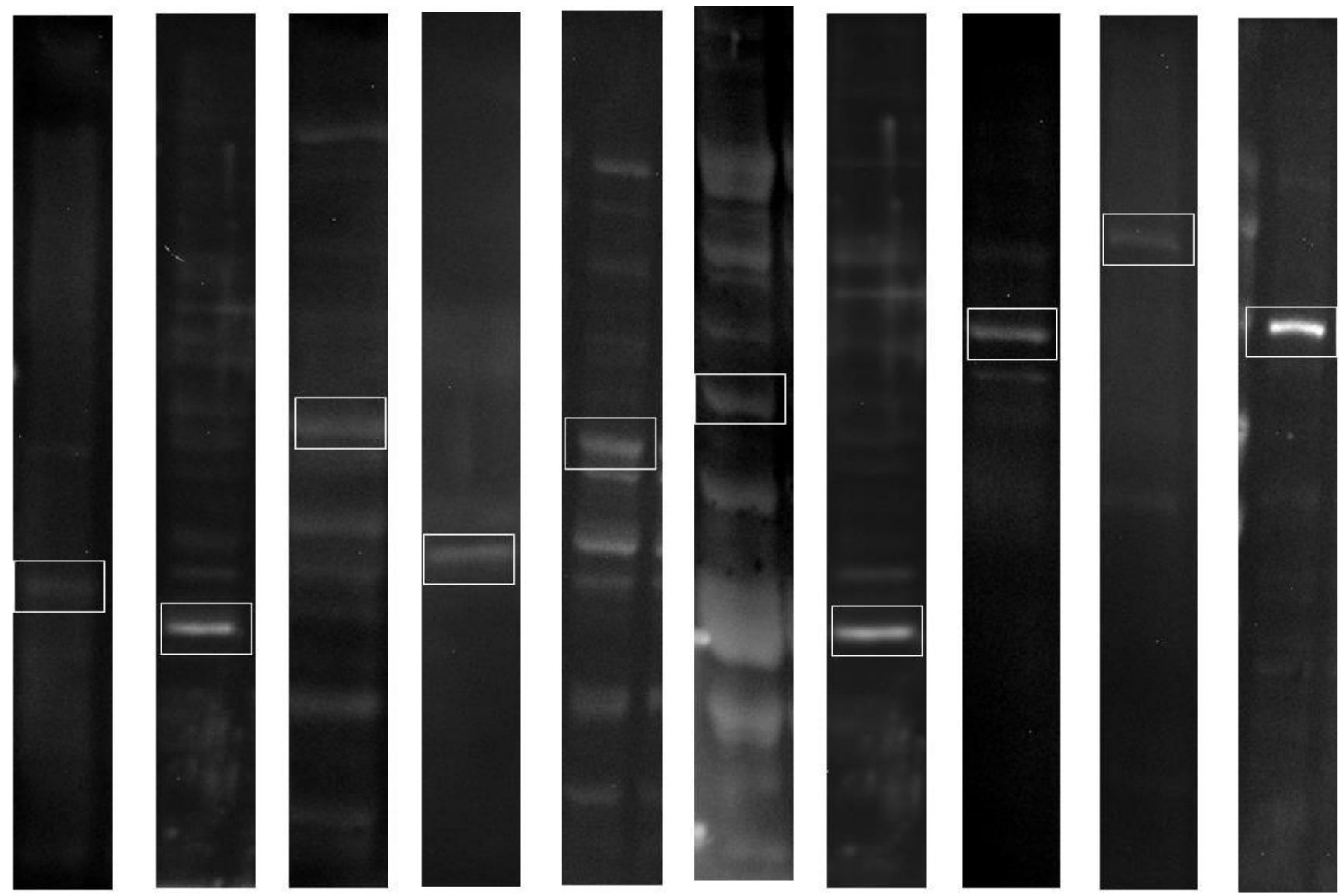

IkB- $\alpha$

P65

P50 -

C-IAP

$\mathrm{Bcl}-\mathrm{xL}$

$\mathrm{Bcl}-2$

$\mathrm{Mcl}-1$

STAT3

(Tyr 705)

529) 337)

Figure A1: Figure shows sample lanes from different proteins from the control condition such that each lane represents one gel probed with one target-specific antibody. A band was chosen for quantification if it appeared at the correct molecular weight, if it was sufficiently separated from other cross reacted bands and if the positive control (taken from the mammalian species Ictidomys tridecemlineatus) appeared at the same molecular weight. 